\title{
Review and Identification of DOE Laboratory Technologies For Countermine/Unexploded Ordnance Detection
}

October 31, 2001

Prepared by

Cyrus M. Smith 


\section{DOCUMENT AVAILABILITY}

Reports produced after January 1, 1996, are generally available free via the U.S. Department of Energy (DOE) Information Bridge:

Web site: http://www.osti.gov/bridge

Reports produced before January 1,1996, may be purchased by members of the public from the following source:

National Technical Information Service

5285 Port Royal Road

Springfield, VA 22161

Telephone: 703-605-6000 (1-800-553-6847)

TDD: 703-487-4639

Fax: 703-605-6900

E-mail:info@ntis.fedworld.gov

Web site: http://www.ntis.gov/support/ordernowabout.htm

Reports are available to DOE employees, DOE contractors, Energy Technology Data Exchange (ETDE) representatives, and International Nuclear Information System (INIS) representatives from the following source:

Office of Scientific and Technical Information

P.O. Box 62

Oak Ridge, TN 37831

Telephone: $865-576-8401$

Fax: 865-576-5728

E-mail:reports@adonis.osti.gov

Web site: http://www.osti.gov/contact.html

This report was prepared as an account of work sponsored by an agency of the United States Government. Neither the United States government nor any agency thereof, nor any of their employees, makes any warranty, express or implied, or assumes any legal liability or responsibility for the accuracy, completeness, or usefulness of any information, apparatus, product, or process disclosed, or represents that its use would not infringe privately owned rights. Reference herein to any specific commercial product, process, or service by trade name, trademark, manufacturer, or otherwise, does not necessarily constitute or imply its endorsement, recommendation, or favoring by the United States Government or any agency thereof. The views and opinions of authors expressed herein do not necessarily state or reflect those of the United States Government or any agency thereof. 


\section{REVIEW AND IDENTIFICATION OF DOE LABORATORY TECHNOLOGIES FOR} COUNTERMINE/UNEXPLODED ORDNANCE DETECTION

Cyrus M. Smith

October 2001

Prepared by OAK RIDGE NATIONAL LABORATORY

P.O. Box 2008

Oak Ridge, Tennessee 37831-6285 managed by

UT-Battelle, LLC for the U.S. DEPARTMENT OF ENERGY under contract DE-AC05-00OR22725 
$\begin{array}{ll}\text { Page } & \end{array}$

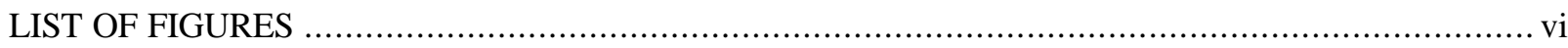

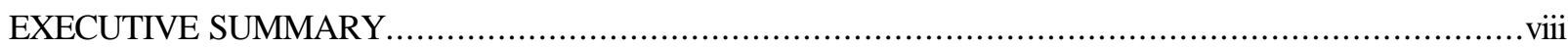

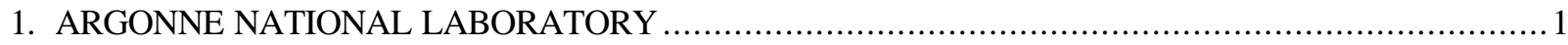

1.1 ACTIVE MICROWAVE IMAGING FOR DETECTION OF NEAR-SURFACE

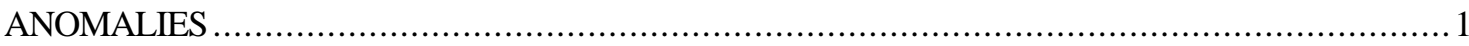

1.2 CHEMICAL-ELEMENT DETECTION BY PENETRATING NEUTRONIC

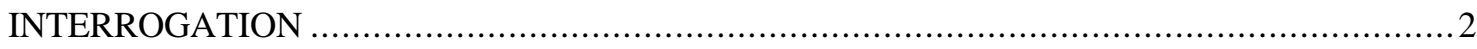

1.3 NEXT-GENERATION BIOSENSING SYSTEM FOR IN SITU DETECTION OF LAND-

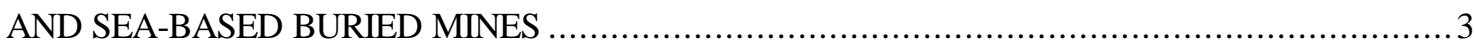

1.4 ECONOMIC, DISPOSABLE, MICROELECTROMECHANICAL LAND MINE

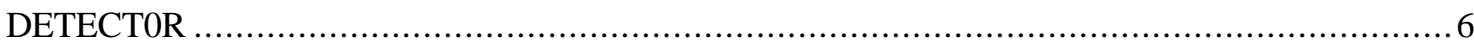

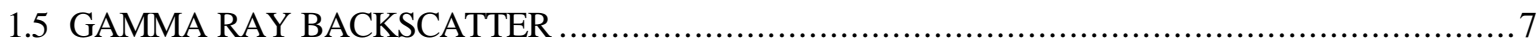

1.6 ECONOMIC, MEMBRANE ENHANCED ION-MOBILITY LAND MINE DETECTOR...............9

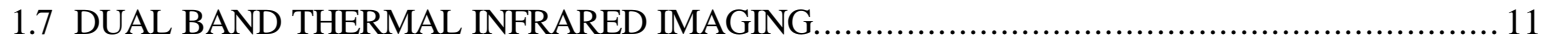

1.8 USE OF MULTISPECTRAL INFRARED IMAGING FOR WIDE AREA SURVEY AND

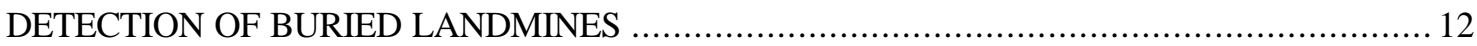

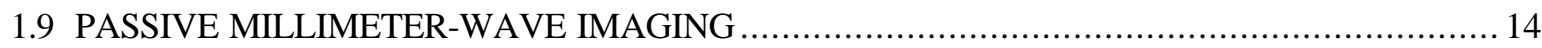

1.10 ANALYSIS OF NUCLEAR MINE-DETECTION TECHNOLOGIES ................................ 16

1.11 ACOUSTIC METHODS FOR LAND MINE DETECTION.............................................. 16

1.12 SAW AND FPW CHEMICAL SENSOR ARRAYS FOR AIR AND GROUND-WATER

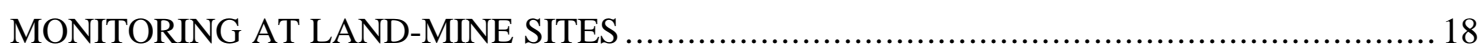

1.13 SWITCHABLE RADIOACTIVE NEUTRON SOURCE …........................................ 20

1.14 MICROWAVE IMAGING WITH SELECTIVE CHEMICAL OR BIOLOGICAL ADSORPTION TECHNIQUE ............................................................................... 21

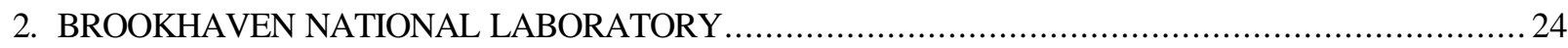

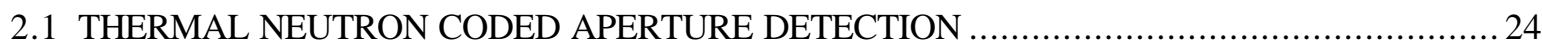

2.2 DETECTION OF LAND MINES USING ELASTIC WAVE SCATTERING (EWS)................ 24

2.3 RF DETECTION AND NEUTRALIZATION OF LAND MINES ….............................. 26

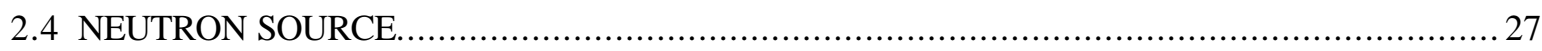

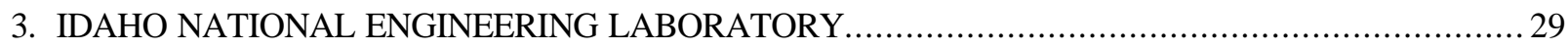

3.1 EXPLOSIVES DETECTION UJSING ION MOBILITY SPECTROMETRY .......................29

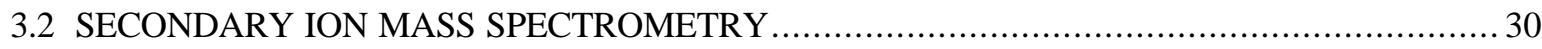

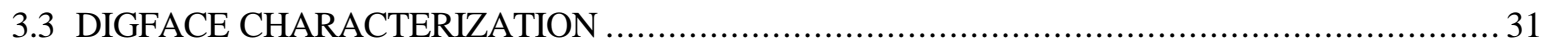

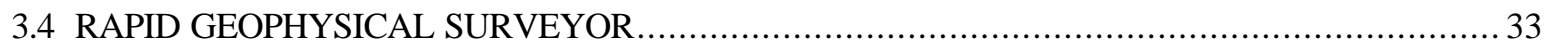

3.5 VERY-EARLY-TIME ELECTROMAGNETIC SYSTEM............................................. 34

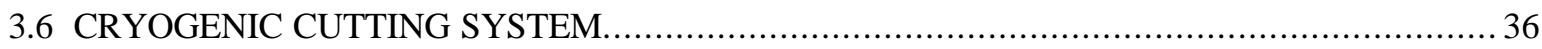

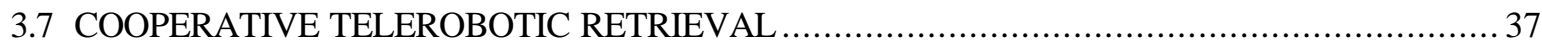

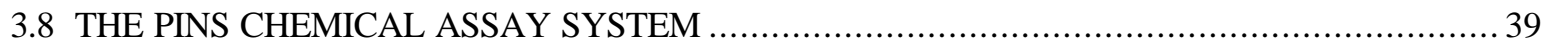

4. LAWRENCE BERKELEY NATIONAL LABORATORY ....................................................... 41

4.1 GAMMA-2N GENERATION OF E+E- ANNIHILATION RADIATION FROM NITROGEN...... 41 
4.2 MINE DETECTION AND IDENTIFICATION WITH A DUAL ELECTROMAGNETIC

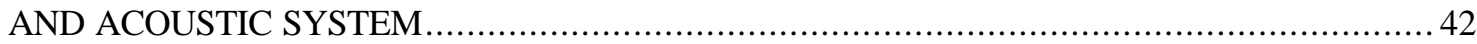

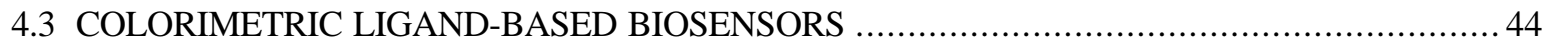

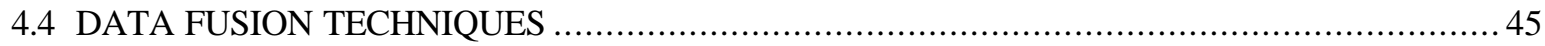

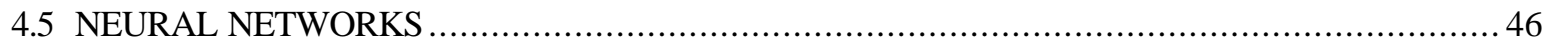

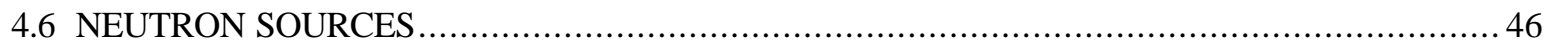

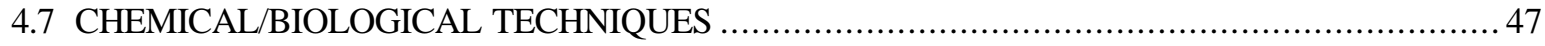

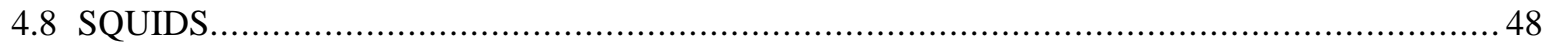

4.9 A D-D NEUTRON SOURCE FOR MINE DETECTION USING THERMAL NEUTRON

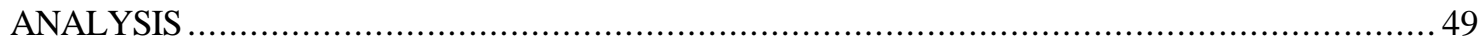

4.10 MINE DETECTION WITH AN RF ELECTROMAGNETIC SYSTEM ................................ 50

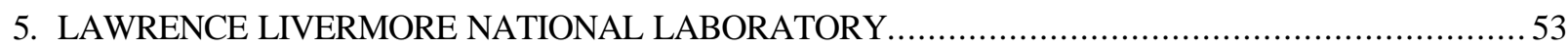

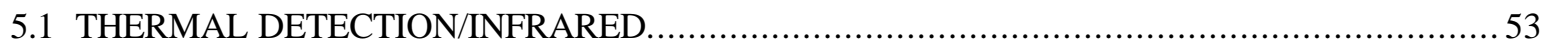

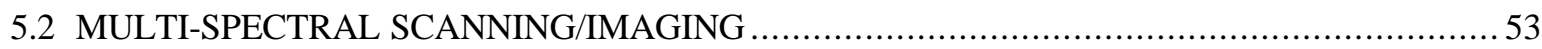

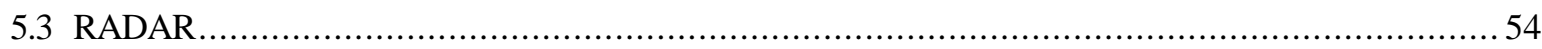

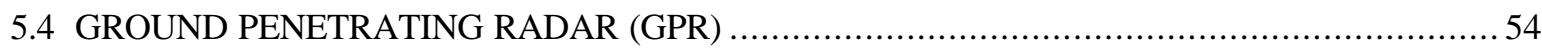

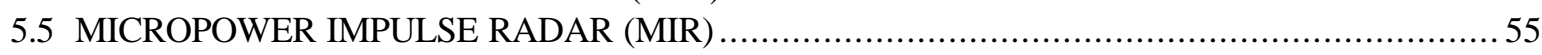

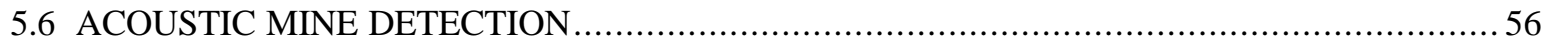

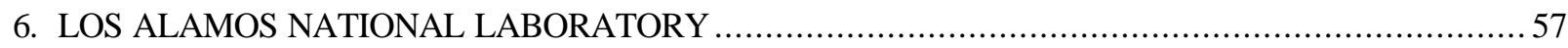

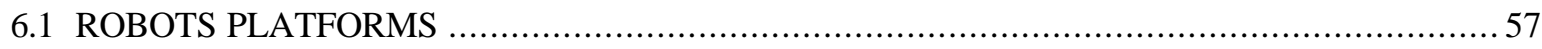

6.2 NOVEL METHODS FOR NEUTRON-INDUCED-GAMMA DETECTION OF LAND

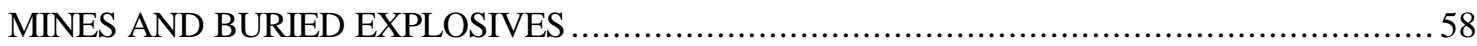

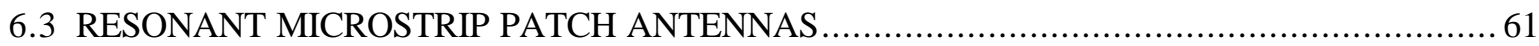

6.4 COMPLETELY PASSIVE LOCATION AND CHARACTERIZATION OF LAND MINES WITH SUPERCONDUCTING QUANTUM INTERFERENCE DEVICE (SQUID) SENSORS63

6.5 ULTRASENSITIVE MINIATURIZED SENSOR SYSTEM FOR DETECTION OF UXO .......... 65

6.6 UXO AND LAND MINE DETECTION USING CHEMICAL MICROSENSORS BASED

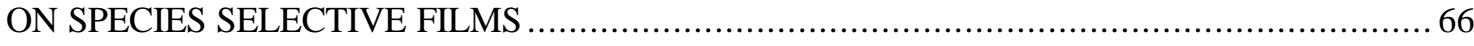

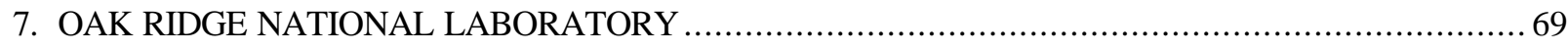

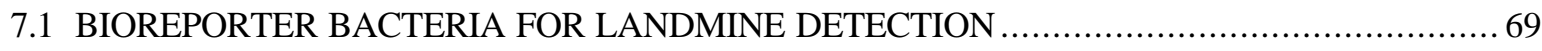

7.2 QUADRUPOLE RESONANCE FOR MINE DETECTION ......................................... 74

7.3 TRACE CHEMICAL DETECTION USING DIRECT SAMPLING ION TRAP MASS

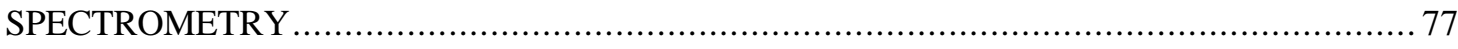

7.4 ANTIPERSONNEL LANDMINE DETECTION USING NANO EXPLOSIONS ON

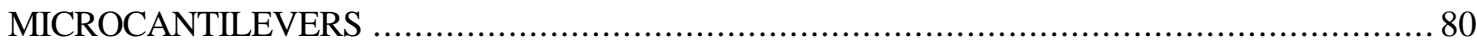

7.5 MINE DETECTION SYSTEM: MOBILITY PLATFORM AND SYSTEMS

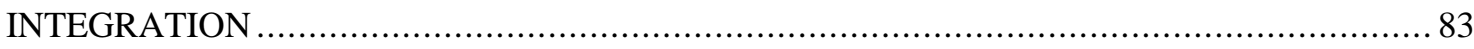

7.6 ADVANCED SIGNAL PROCESSING AND DATA FUSION FOR MINE DETECTION ............ 87

7.7 CALIFORNIUM-252 BASED NEUTRON INTERROGATION SYSTEM FOR THE DETECTION

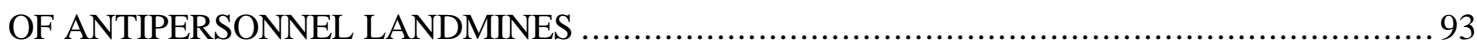

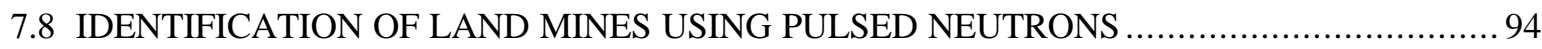

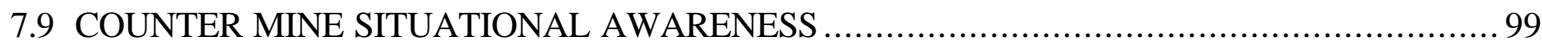

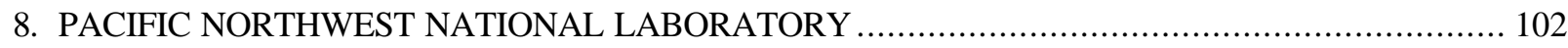

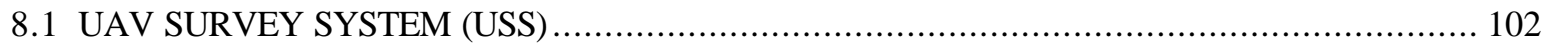

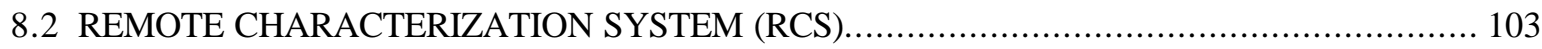

8.3 NUCLEAR METHODS FOR DETECTION OF MINES AND UNEXPLODED ORDNANCE.... 104 
8.4 ULTRA-SELECTIVE VAPOR “SNIFFER” FOR GAS PHASE CHEMICAL

MEASUREMENTS

8.5 ARTIFICIAL NEURAL NETWORKS .....

8.6 GENETIC ENGINEERING OF ORGANISMS FOR HIGHLY SELECTIVE DETECTION OF MINES AND UNEXPLODED ORDNANCE 107

8.7 THE DETECTION OF MINES AND UNEXPLODED ORDNANCE (UXO) VAPORS AND PARTICULATES USING AN INTEGRATED MICROTECHNOLOGY PRECONCENTRATOR AND SENSOR SYSTEM 108

8.8 UWB RADAR HOLOGRAPHIC IMAGING SYSTEM FOR BURIED METAL AND PLASTIC MINES 112

8.9 DETECTION OF LAND MINES AND UNEXPLODED ORDINANCE USING AN ENZYMATIC CHEMICAL REACTION BASED SENSOR 114

8.10 SMART TERRAIN PC-BASED TERRAIN VISUALIZATION CAPABILITY 115

9. SANDIA NATIONAL LABORATORY 117

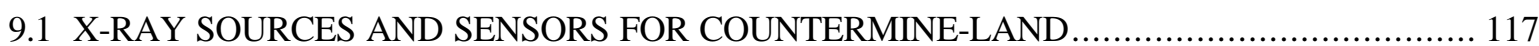

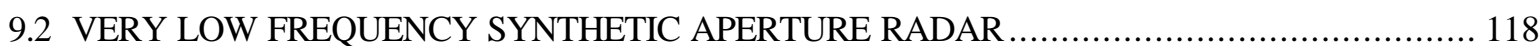

9.3 HIGH PERFORMANCE CHEMICAL ANALYSIS SYSTEMS INTEGRATED ON A CHIP. 119

9.4 SIGNAL AMPLIFICATION BY CHEMICAL REACTION 120

9.5 HAZARDOUS MATERIAL REMOVAL USING A HIGHLY DEXTEROUS, REMOTE MOBILE MANIPULATOR

9.6 ION MOBILITY SPECTROMETRY (IMS) FOR CLASSIFICATION OF UXO

9.7 USE OF RIGID POLYURETHANE FOAMS (RPF) IN MINE COUNTERMEASURES, CROSSING BEACH BARRIERS DURING AMPHIBIOUS ASSAULT, AND ROAD IMPROVEMENT AND REPAIR

9.8 CODED APERTURE X-RAY LAND MINE IMAGING

9.9 TRANSPORTABLE, CONTAINED, BATCH DESTRUCTION SYSTEM FOR UXO

9.10 AUTOMATED HAZARD SEPARATION FOR MUNITION DEMILITARIZATION

9.11 CHEMICAL TAGGING AND SIGNAL TRANSDUCTION

10. SAVANNAH RIVER TECHNOLOGY CENTER

10.1 BIOLOGICAL UXO INDICATOR 


\section{LIST OF FIGURES}

Figure

Page

1. Near scale schematic diagram of the proposed Autonomous Microelectromechanical Land Mine Detector

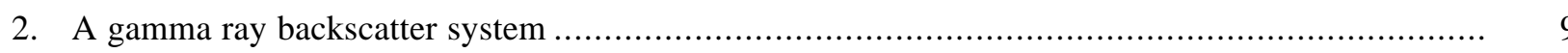

3. Larger than scale diagram of the proposed Economic, Membrane Enhanced Ion-Mobility Land Mine Detector

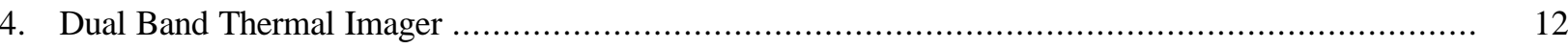

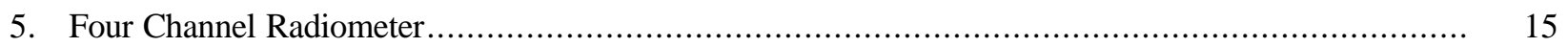

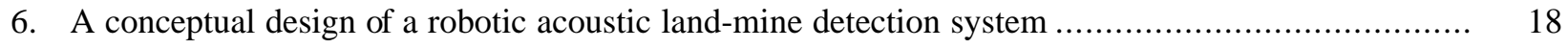

7.A. The basic configuration of a surface-acoustic-wave chemical sensor ................................. 20

7.B. The basic design of a flexural-plate-wave chemical sensor ....................................... 20

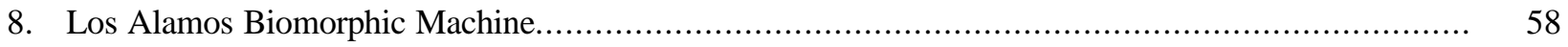

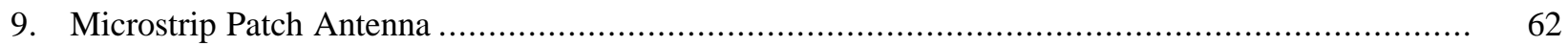

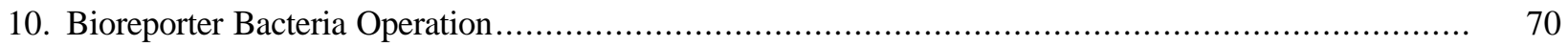

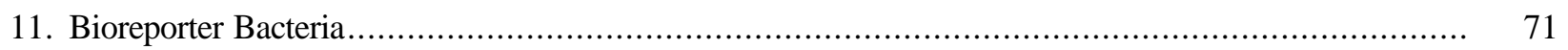

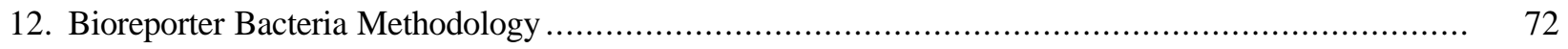

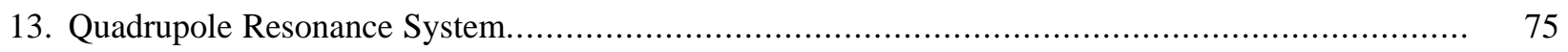

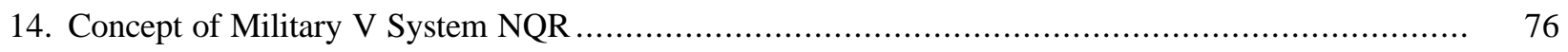

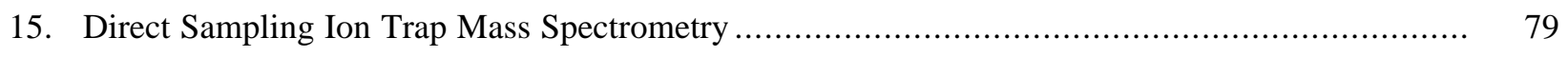

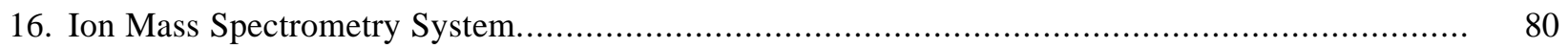

17. Landmine Detection by Nanoexplosion Microsensors ................................................ 82

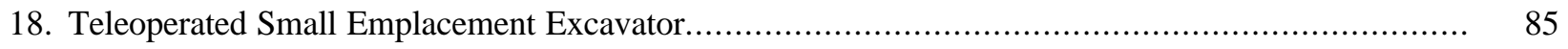

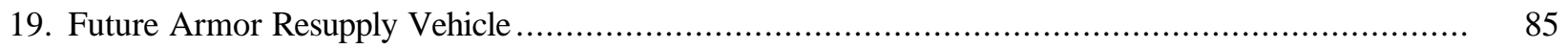

20. DOE/DOD Remote Characterization System .......................................................... 86

21. Robotics \& Process Systems Division's highbay facility ................................................. 87 
22. Hand probing for land mines

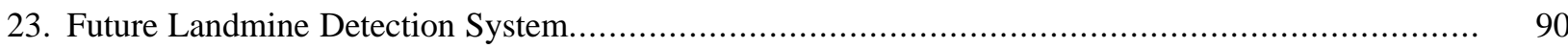

24. With one cycle of discrete Fourier processing, a-11 DB S/N signal can be readily identified ........... 91

25. With one cycle of wavelet/curve fit processing, a -27 DB S/N signal can be readily identified ........ 91

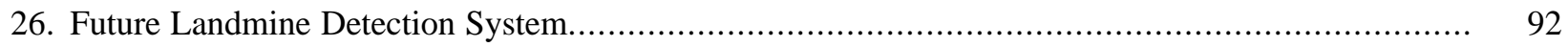

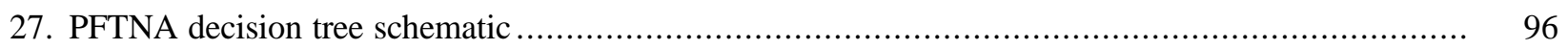

28. Experimental gamma ray spectra of mine-like objects............................................

29. Schematic view of proposed vehicle mounted PFTNA system ....................................... 98

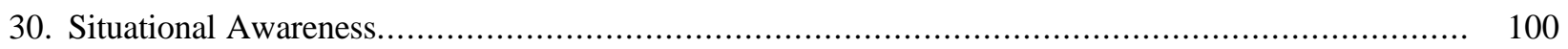


EXECUTIVE SUMMARY

\section{Review \& Identification of DOE Laboratory Technologies For Countermine/Unexploded Ordnance Detection}

\section{DOE Laboratory Participants}

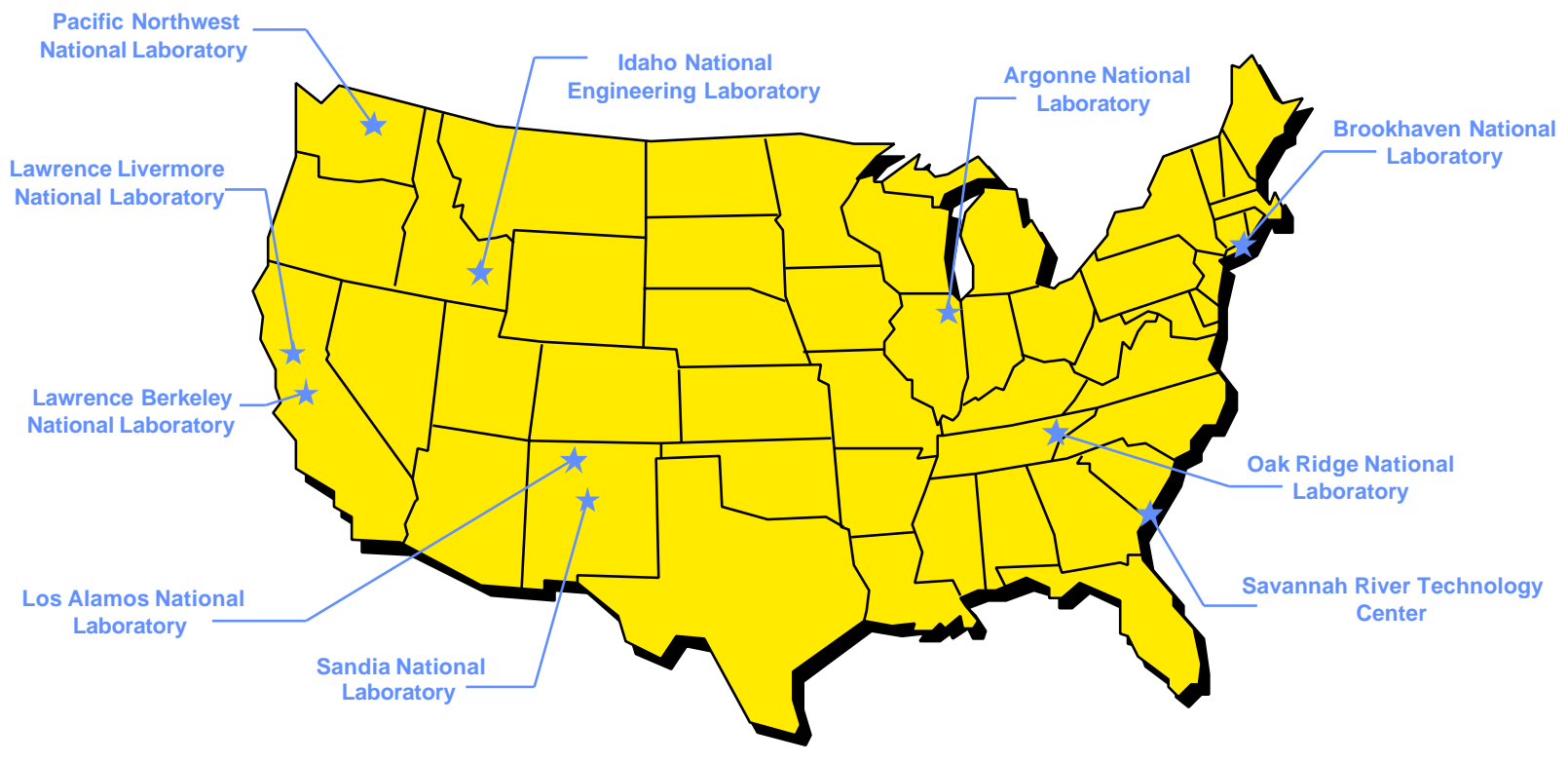

Several Department of Energy (DOE) laboratories have worked and/or are working on technologies that are applicable to the detection of landmines and/or unexploded ordnance. This report is a compilation of technical summaries for many of these technologies. For additional information on any technology, appropriate points of contact are provided for each technology. 


\section{ARGONNE NATIONAL LABORATORY}

\subsection{ACTIVE MICROWAVE IMAGING FOR DETECTION OF NEAR-SURFACE ANOMALIES}

\section{Technical Contacts}

S. Bakhtiari and A. C. Raptis

Energy Technology Division

Argonne National Laboratory

9700 S. Cass Ave

Argonne, IL 60439

Phone: (708) 252-8982 (5907)

Fax: (708) 252-3250

E-mail: sasan_bakhtiari@qmgate.anl.gov

\section{Description of Technology}

Microwave noncontact probing techniques offer high resolution and sensitivity for examination of a wide range of dielectric media. Due to versatility of such systems, their range of application extends from nondestructive evaluation of advanced dielectric composite materials to medical imaging area. Advancements in the microwave solid-state technology and real-time computer based imaging have further increased the applicability of microwave sensors for real-time insitu applications. Feasibility of high-resolution microwave imaging using fixed frequency coherent sources have been demonstrated to some extent in the past. Research on new wavefront processing algorithms is currently being pursued to enhance real-time image reconstruction for detection of buried objects. With the component dimensions being in the order of operating wavelength at microwave frequencies ( $\mathrm{cm}$ range), compact microwave imaging systems would inherently offer great potential for such applications. Further, with the antenna beamwidth being inversely proportional to the operating frequency, higher microwave frequencies (i.e. shorter wavelengths) inherently allow for finer resolution probing given that the dielectric losses inside the material medium allow for detection of backscattered signal. The penetration of microwave radiation inside most nonconducting, dry, solid media is suitable for detection of buried objects at multiple wavelengths beneath the surface which is also well within the detection range of solid state receivers. The upper limit on the usable frequency is dictated by material complex dielectric properties and the state of technology. Quasi far-field lens antennas using focused beams may be used for fine resolution measurements to be made at any distance from the material surface under test. Such sensors are ideally suited for insitu measurements in harsh environments.

Some representative c-scan images have been made with ANL's benchtop millimeter wave reflection/transmission sensor for some dielectric composite samples with artificially embedded defects. The samples consisted of plates of Kevlar-fiber-reinforced-epoxy composite which contained subsurface voids and disbonds. The low-power W-band system which incorporates Gaussian optic lens antennas allows for high resolution inspection of materials with minimal diffraction losses. With minor modification of the system, such techniques as synthetic aperture imaging may be readily implemented for wide area monitoring applications. 


\section{Technical Status/Maturity}

Research on microwave imaging done on various dielectric materials at the millimeter wave laboratory of ANL has proven the immense potential of high frequency electromagnetic radiation for non-contact probing applications. The currently operational W-band imaging sensor may be used for polarization dependent measurements of near-surface anomalies. Such high resolution, non-contact sensor technology utilizes lowpower solidstate sources and microwave monolithic integrated circuit (MMIC) technology. No coupling medium is necessary and the system is well suited to operate in harsh environments (i.e. presence of heat, stress, airborne particles, etc.). The millimeter wave group of the ET division at ANL have extensive experience in the area of NDE sensor technology and are equipped with facilities to carry out research within the microwave spectrum. They have been involved in the past with implementation of on-line microwave sensors for real-time inspection applications.

\subsection{CHEMICAL-ELEMENT DETECTION BY PENETRATING NEUTRONIC INTERROGATION}

\section{Technical Contacts}

Dr. Alexander DeVolpi

Phone: (630) 252-4598

Fax: (630) 252-3078

E-mail: devolpi@td.anl.gov

Dr. Charles Dickerman

Phone: (630) 252-4622

Fax: (630) 252-4780

E-mail: dickerman@nutron.re.anl.gov

Because of variability in the surrounding medium, buried mines are difficult to detect with high confidence unless unique characterization of explosive content is achieved. Detection with minimal personnel risk might be best accomplished with a simultaneous combination of several synergistic technologies. Non-nuclear techniques can be highly efficient at detection of buried anomalies. However, identifying these anomalies as explosive mines with sufficiently high probability for humanitarian de-mining continues to be a technical challenge. Nuclear techniques, which can identify chemical-element compositions, are likely to contribute to mine detection in three ways: (1) explosives recognition in anomalies detected by non-nuclear techniques, (2) characterization of the medium that surrounds anomalies, thus augmenting the capability of non-nuclear techniques, and (3) deep penetration into the ground, thereby complementing shorter-range non-nuclear methods.

A fieldable nuclear technique that could assist in these mine-detection goals is the associated-particle sealedtube neutron generator (APSTNG). Compared to other techniques, it would have advantages in detecting deeply buried anti-tank mines of any construction. The APSTNG produces penetrating neutrons of $14 \mathrm{MeV}$. Neutron inelastic scattering and capture would provide ratios of $\mathrm{H}, \mathrm{C}, \mathrm{O}$, and $\mathrm{N}$ and other elements to reasonable depths for detection of all types of buried mines. Three-dimensional localization of explosives is also a capability of the APSTNG that would be valuable in mine detection. Because of its efficient use of neutrons for penetrating interrogation, the APSTNG offers comparatively low radiation hazard to operators while definitively identifying anomalous buried objects. 


\section{Technical Status}

Laboratory and field demonstrations of ANL's APSTNG detection system (including neutron generator, power supplies, electronics, and analysis software) have previously been conducted under DOE funding to detect and identify diverse objects, including chemical munitions, fissile materials, and buried substances. A new generation of APSTNG technology is due to be delivered to Argonne by the end of 1996. The primary task remaining would be to package a system for field testing. Collaboration is under way with Russian institutes for application of APSTNG technology to de-mining operations underway in Russia.

\subsection{NEXT-GENERATION BIOSENSING SYSTEM FOR IN SITU DETECTION OF LAND- AND SEA- BASED BURIED MINES}

\section{Technical Contacts}

\author{
S. Ahuja, Ph.D \\ Energy Technology Division \\ ANL \\ Phone: (708) 2528851 \\ Fax: (708) 2523250 \\ E-mail: sanjay@anl.gov
}

A. C. Raptis, Ph.D.

Energy Technology Division

ANL

Phone: (708) 2525930

Fax: (708) 2523250

E-mail: ac_raptis@qmgate.anl.gov

\author{
M. H. Bhattacharyya, Ph.D. \\ Center for Mechanistic Biology and \\ Biotechnology \\ ANL \\ Phone: (708) 2523923 \\ Fax: (708) 2523387 \\ E-mail: bhatt@anlbem.bim.anl.gov \\ T. Lavin, M.D. \\ Cardiovascular Center \\ Cornell University Medical Center, NY \\ Phone: (908) 7564006 \\ Fax: (908) 7557266 \\ E-mail: tlavin@gensis.co
}

\section{Description of Technology}

In the present state of technology, sensing systems for mine detection provide information at only a gross level of control and are not necessarily optimized. The work on land mine detection has mainly been performed on the soil surface with overwhelming equipment limitations for detection of deeply buried land and sea mines. Identification of buried land mines under dry or aqueous environment cannot be precisely conducted by today's technology due to the absence of highly resolving and sensitive chemical sensing systems. The challenge exists when it becomes necessary to quantitatively and accurately trace deeply buried land or sea mines and to isolate lethal mines from other buried non-lethal objects.

The main thrust of the proposal is to identify deeply buried objects, trace the objects and isolate the lethal land mines from non-lethal objects. The work will concentrate on process monitoring and control to devise a sophisticated sensing system. A biodegradable penetrating catalyst blanket will be deposited on the area under suspicion of having land mines. This catalyst will aid in bringing the information from soil depths to the surface. An external electric field will be induced to the buried objects and the pronounced changes in the electric field will be propagated by the catalyst, through a change in the state of the molecules, to the abovesurface microsensing system. 
The above-surface microsensing system will consist of an integrated micromachined sensor coupled with a specific biological recognition unit which will detect changes in the state of the catalyst, thereby aiding in tracing the buried objects. The change in the state of the catalyst to the above-surface sensing system will give rise to a frequency change which will be different depending upon the shape of the buried objects, thereby allowing accurate tracing of buried objects. The buried objects will be traced and identified with good precision since subtle changes in frequency of the microsensor can be detected. The extreme sensitivity of the design of the proposed microsensing system will allow instant isolation and easy detection of deeply buried mines which has not been possible with the present sensing methods.

\section{Technical Progress And Specific Accomplishments}

The present focus of the research has been in the design and manufacture of a surface acoustic wave (SAW) microsensor whose functionality lies in the change in a property of the device surface resulting in a variation or attenuation of the acoustic wave velocity detected by a change in the induced frequency. For sensing, the interaction mechanism of the absorbed or adsorbed molecules with the probing SAW has been the variation in electrical conductivity. The basic elements of the currently devised chemical sensor consists of a substrate, transmitting and receiving interdigital transducers (IDTs), and chemically sensitive films deposited on the top surface. The IDTs launch and receive the SAWs and together with an amplifier form a feedback oscillator whose output frequency provides a measure of the electric conductivity of the chemically sensitive film. Initial tests are underway to test the functionality of the SAW microsensor in terms of electric conductivity changes.

In the future, the output of the device will be compared with that of a reference sensor, the circuit connections with the sensor will be made for electrical conductivity measurements and the microsensor assembly will be tested. The response of the coated film will be tested and the number of factors perturbing the surface wave velocity will be determined. A relationship between the temperature coefficient of the substrate with respect to the frequency shift will be devised to better understand the temperature change of the microsensor and the temperature compensation which reduces thermal drift. Experiments will be performed to increase sensitivity and response time with respect to the film thickness and the results will be compared with resistance-measuring sensors.

\section{Justification}

The advantages of the proposed microsensor are the excellent characteristics, sensitivity, and resolution of identifying deeply buried objects that are potentially hazardous to humans and which deteriorate living conditions. The self contained aspect of the proposed sensing system will enable field measurements to be performed without the need for sophisticated laboratory equipment. Further, the biodegradable biocatalyst will be air-spread keeping human interaction at the minimum. The unique characteristics of the proposed microsensor system, such as on-line, non-optical, direct read-out, field usable, self contained, and micromachined, are not presently available and make tremendous enhancements in the current state of technology. Utilization of the intelligent microsensors will decrease the cost of manufacturing and energy consumption while saving time leading to increased robustness of sensor measurements and ultimately to increased accuracy in measurement. 


\section{Current And Pending Support}

- Laboratory directed research and development funds of $\$ 400 \mathrm{~K}$ for two years to develop a smart, micromachined sensing system for simultaneous measurement of temperature, pressure and strain.

- A pending proposal, expected to be funded in July 1996 for $\$ 900 \mathrm{~K}$ for three years, for microfabrication of a sensor based on the Fabry-Perot interferometry techniques.

- A pending proposal, expected to be funded by September 1996

- $\quad$ NIH RO-1 Mechanism of Thyroid Action, Fund year 1. July 1989 \$546,458

- Karo-Bio AB, Research Contract, 1988-1989 \$257,000

- Clinical Investigator Award 1984-1987 \$290,000

- University of California React grant

- American Thyroid Foundation grant

- University of California Shared Equipment grant

\section{Facilities And Resources}

Numerous micromachining technology projects are being carried out at Argonne National Laboratory. A micromachined sensor for concurrent sensing of pressure, temperature and strain is being devised. The Sensors, Instrumentation \& Non-Destructive Evaluation (SINDE) section has extensive experience in sensor development and instrumentation by utilizing ultrasonic techniques. An ultrasonic viscometer was developed to measure density and viscosity of fluids. Extensive experience exists in applying the Lamb wave technique to determine material properties, defects, fiber orientation in solids. Microstructural evolution during processing of metal parts has been evaluated under varying processing conditions. Currently, effort is underway to monitor adhesive curing and determine the thermophysical properties of liquids, gels, and residuum. An ultrasonic flow imaging system is being developed to monitor flow characteristics. The Energy Technology Division at Argonne National Laboratory has extensive experience in sensor technology. Various kinds of sensors, e.g. millimeter wave, surface acoustic wave, leak detection, temperature, and flow and chemical sensors, have been devised within the Division. A new micro-mass spectrometer now under development has many potential environmental applications. Nuclear magnetic resonance concepts are also being applied to analyze various chemicals.

A large optics facility in the division is used to enable material analysis, chemical vapor deposition, coatings, material evaluation, and enhancements. The staff has extensive experience in radiographic and ultrasonic detection methods used in detecting flaws in submicrometer films. The extensive facilities for property and fracture testing, nondestructive evaluation, and computational analysis are applicable to the production and design optimization of sensors. Advanced sensor and instrumentation techniques are being applied to characterize the nonlinear effect of adhesive and adherent materials and to monitor adhesive curing. Extensive experience exists in the area of ultrasonics to determine properties, defects, fiber orientation in particulate- and fiber-reinforced metal-matrix composites, ceramics, etc. Ultrasonic microscope systems, acoustic-ultrasonic testing systems, infrared imaging systems, millimeter wave imaging systems, and 3-D X-ray high resolution tomographic image systems are available for microstructural and adhesive joint evaluation. LIGA equipment which can be used to produce molds for the fabrication of micromachined components is available at Argonne. In addition, facilities at Northwestern University, especially the Materials Processing and Crystal Growth Facility, may be used. These facilities include capabilities such as photolithography, chemical vapor deposition and furnaces, 
etching, metallization, surface and optical characterization equipment, electrical characterization equipment, dicing and lead attachments, etc.

Optimization of interaction between protein and ligand will be undertaken using existing capabilities within the Center for Mechanistic Biology and Biotechnology. These capabilities include x-ray diffraction systems for determination of protein structure, molecular biology laboratories for the cloning, expression, and site-specific modification of proteins, as well as computational systems for modeling the interactions between a protein and ligand and to predict the consequences of protein modification on that interaction. These capabilities have been well established in studies of various proteins including antibody components, enzymes, and the photosynthetic reaction center. Sufficient facilities for protein production, silane chemistry, polymer coating etc exist at Cornell University Medical Center. Large scale laboratory equipment such as semi-automatic pipettors, shaking tables, low pressure chromatography equipment and chart recorders are available. Vented laboratory ovens, required for the proposed work, and additional laboratory items are available on-site.

\subsection{ECONOMIC, DISPOSABLE, MICROELECTROMECHANICAL LAND MINE DETECTOR}

\section{Technical Contacts}

\section{S. L. Dieckman}

Phone: (708) 252-5628

Fax : (708) 252-3250

E-mail: dieckman@anl.gov

\section{A. C. Raptis}

Phone: (708) 252-3930

Fax: (708)-252-3250

E-mail: ac_raptis@qmgate.anl.gov

\section{Description of Technology}

The objective of this program is develop an intelligent, sensitive, accurate, disposable (low-cost), mass produced, "smoke detector - like" chemical sniffer. The device will be used as a single-man-portable mine detector or integrated with additional units onto a mobile platform. This sensor system will be based upon a micromachined time-of-flight mass spectrometer technologies. This technology is capable of sensing to parts-per-billion (ppb) levels over a broad range of volatile organic chemicals (VOCs), semivolatile organics (SVOCs), and volatile inorganics. Furthermore, this device can be highly specific when outfitted with a selective inlet membrane. Specifically, the sensor will be composed of mass produced micro-machined components that are incorporated into a miniaturized $(10 \mathrm{~cm})$ time-of-flight mass spectrometer. The entire spectrometer will weigh approximately 1.5 pounds and be the size of a conventional smoke detector (Fig. 1). The sensitivity and resolution of the spectrometer are projected to be $\mathrm{ppb}$ for a $15 \mathrm{sec}$ scan, with high resolution (sub atomic mass unit/charge amu/z) through mass/z 300 . The device will be preprogrammed with heuristics that provide intelligent operation and data analysis. The research associated with this program will include (1) the development of the micromachined electron and ion optics, (2) the integration of the sub-components into a completed sensor, and (3) the development of the artificial intelligence for automated operation and verification of the sensor's efficacy. 


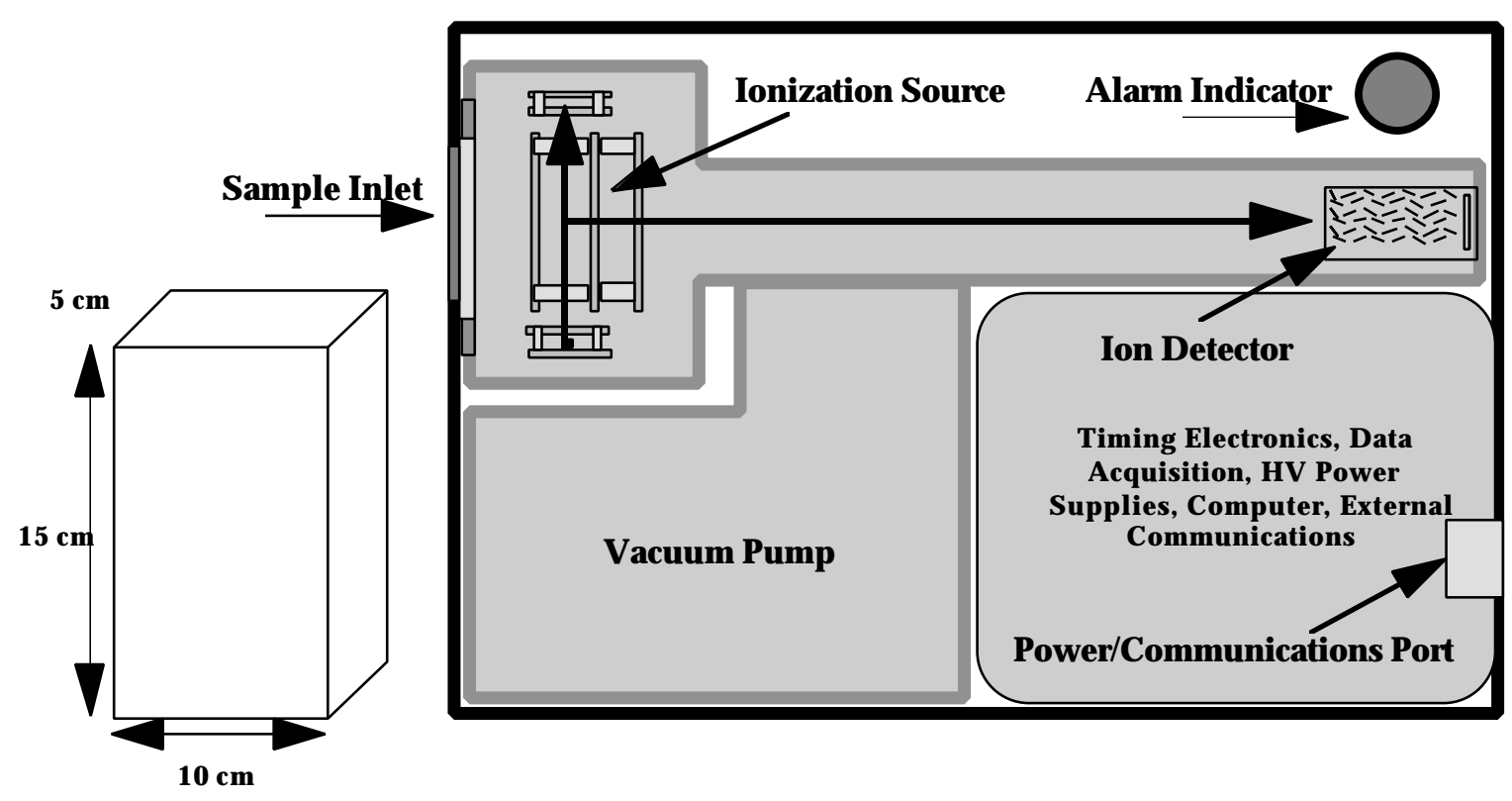

Fig. 1. Near scale schematic diagram of the proposed Autonomous Microelectromechanical Land Mine Detector.

\section{Technology Maturity}

This technology will be sufficiently mature as to allow system engineering by the year 1998. ANL currently has funding from DOE-Office of Nonproliferation and National Security for a hand portable photoionization mass spectrometer designed from conventional components. Additionally, we are in the process of signing a small subcontract from NASA-JPL for funding to perform a feasibility study on miniature time-of-flight mass spectrometers for space-based applications.

\section{Technology Significance}

The device will be capable of being pre-programmed for multiple in-field mine detection operations. Other DOD applications of this sniffer technology will include, (1) fast personal chemical weapon monitoring,

(2) battlefield perimeter monitoring, and (3) anti terrorism/explosive monitoring systems for use around installations and field bases. Civilian applications may include process sensing and control, environmental monitoring, space based sensors (e.g., miniaturized sensors for NASA's New Millennium program), and applications to civilian aviation and law enforcement and counter terrorism.

\subsection{GAMMA RAY BACKSCATTER}

\section{Technical Contacts}

J. G. Sun

Energy Technology Division

Argonne National Laboratory

Phone: (630) 252-5169

Fax: (630) 252-4798

jg_sun@qmgate.anl.gov 
A. C. Raptis

Energy Technology Division

Argonne National Laboratory

Phone: (708) 252-3930

Fax: (708) 252-3250

E-mail: ac_raptis@qmgate.anl.gov

\section{Description of Technology}

The objective of this proposal is to build and test a vehicle based gamma ray backscatter detection system for screening areas laden with antipersonnel and antitank mines. The technique is based on the characteristics of interaction of gamma ray with matter. Depending on the energy level of the gamma ray, one of the following three mechanisms is dominant in the interaction: the photoelectric effect, Compton scattering, and pair production. The backscattered energy levels and intensities are dependent upon the material composition and density. These characteristics may be used to detect land mines because of the difference in materials and in density of the land mines compared with the surrounding ground soil. Furthermore, because of the high penetration power of the gamma radiation, it is possible to detect land mines deeper under the surface.

A gamma-emitting radioisotope provides the source of gamma radiation into the ground soil. The backscattered rays are collected by the scintillation detectors which may be strategically arranged for screening of a wide area. The electronics utilize the gamma ray counting method. Depending on the particular energy levels associated with the mine materials, the discriminator can be adjusted to pass through only those signal pulses corresponding to the desired energy levels. The count rates of the detectors are related with the material density. They are continuously recorded and analyzed by an on-line computer. An abrupt change in the count rates would indicate a land mine or foreign object in the detection area. With proper selection of the gamma source and the detection system and development of data processing and correlations, positive identification of land mines with high sensitivity is possible.

Argonne National Laboratory has complete knowledge in design and construction of a computer-aided particle tracking facility for application in determining the motion of a radioactive tracer particle in solids fluidized bed systems. The facility employed 16 scintillation detectors which count the gamma photons emitted directly from the radioactive tracer. The detector count rate was sampled in frequencies up to $200 \mathrm{~Hz}$ for several hours. The instantaneous positions of the tracer were calculated and then recorded in the computer in realtime based on previously determined calibration curves between count rate and tracer-detector distance for individual detectors. The facility has been used for fluidized beds as large as 2 feet in diameter with accurate measurement results. 


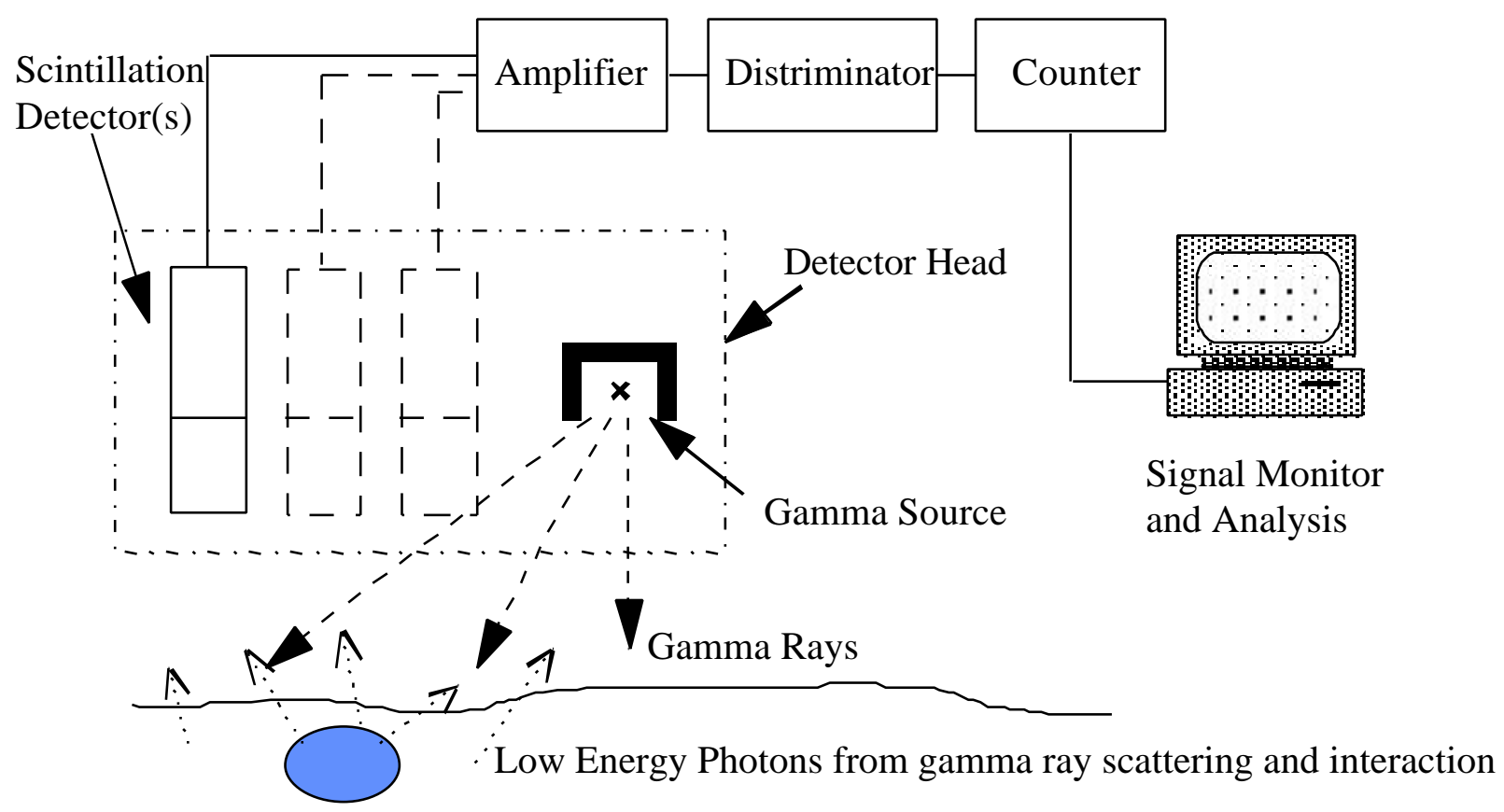

Fig. 2. A gamma ray backscatter system.

\section{Technical Status/Maturity}

Backscatter spectroscopies are mature and commercially available techniques for analyzing material compositions and quantities, e.g., RBS, EDX, etc. The sources include ions, electrons, and X-rays which differ from gamma rays only by energy levels. The detection system illustrated in Fig. 1 has been developed in a university for more than 15 years, and a unit was assembled and installed at a DOE laboratory site. Approximately $\$ 1$ million funding have been provided from NSF, DOE, and other agencies for development and application of this technology. The system consists of low cost equipment readily available commercially. The radiation involved would not be a major health concern because of the low radioactive intensity sources used in the system.

\subsection{ECONOMIC, MEMBRANE ENHANCED ION-MOBILITY LAND MINE DETECTOR}

\section{Technical Contacts}

\section{S. L. Dieckman}

Phone: (708) 252-5628

Fax: (708) 252-3250

E-mail: dieckman@anl.gov

A. C. Raptis

Phone: (708) 252-3930

Fax: (708) 252-3250

E-mail: ac_raptis@qmgate.anl.gov 


\section{Description of Technology}

The objective of this program is develop an intelligent, sensitive, chemical sniffer based upon membrane enhanced ion mobility spectrometry. The device will be used as a single-man-portable mine detector or integrated with additional units onto a mobile platform. This sensor system will be based upon a micromachined ion mobility spectrometer technologies. This technology is capable of sensing to sub partsper-billion (ppm) levels over a broad range of volatile organic chemicals (VOCs), semi-volatile organics (SVOCs), and volatile inorganics. Furthermore, this device can be highly specific when outfitted with a selective inlet membrane. Specifically, the sensor will be composed of mass produced micro-machined components that are incorporated into a miniaturized $(10 \mathrm{~cm})$ ion mobility spectrometer. The entire spectrometer will weigh approximately 0.5 pounds and be the size of a conventional (Fig. 3). The sensitivity of the spectrometer are projected to be sub-ppm for a $5 \mathrm{sec}$ scan. The specificity will be based upon both the mobility of the explosives in the atmosphere and the selectivity of the membrane. The device will be preprogrammed with heuristics that provide intelligent operation and data analysis. The research associated with this program will include (1) the development of the miniaturized components, (2) the integration of the sub-components into a completed sensor, and (3) the development of the artificial intelligence for automated operation and verification of the sensor's efficacy.

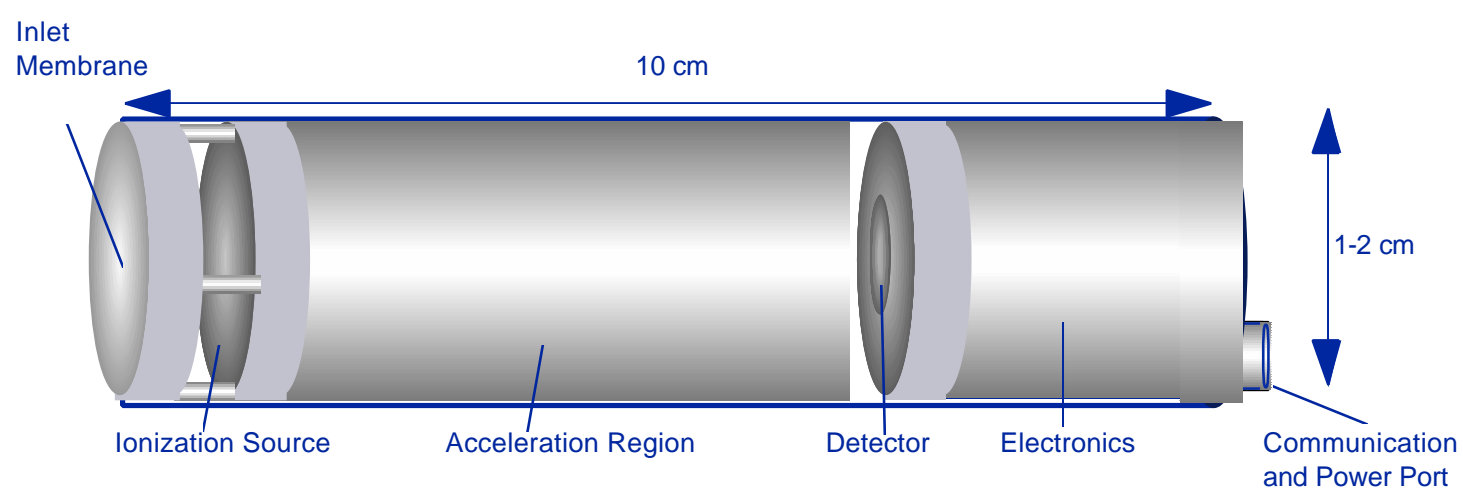

Fig. 3. Larger than scale diagram of the proposed Economic, Membrane Enhanced Ion-Mobility Land Mine Detector.

\section{Technology Maturity}

This technology will be sufficiently mature as to allow system engineering by the year 1998. ANL currently has funding from DOE-Office of Transportation Technologies to develop an ion mobility system that is capable of monitoring ppm organics in exhaust streams.

\section{Technology Significance}

The device will be capable of being pre-programmed for multiple in-field mine detection operations. Other DOD applications of this sniffer technology will include, (1) fast personal chemical weapon monitoring, (2) battlefield perimeter monitoring, and (3) anti terrorism/explosive monitoring systems for use around installations and field bases. Civilian applications may include process sensing and control, environmental monitoring, space based sensors (e.g., miniaturized sensors for NASA's New Millennium program), and applications to civilian aviation and law enforcement and counter terrorism. 


\subsection{DUAL BAND THERMAL INFRARED IMAGING}

\section{Technical Contacts}

N. Gopalsami, J. G. Sun, and A. C. Raptis

Energy Technology Division

Argonne National Laboratory

Phone: (630) 252-5925

Fax: (630) 252-3250

E-mail: gopalsami@anl.gov

\section{Description of Technology}

Thermal infrared imaging is a fairly mature technology that makes use of thermal emissions (blackbody radiation) of objects. The objective of this proposal is to build and test vehicle and/or helicopter-mounted IR imaging systems for fast detection and screening of wide areas laden with antipersonnel and antitank mines. Surface mines can be imaged with high resolution using this technology any time of the day or night. Because of clear object identification, low false positives will be possible with this technology for surface mines. Detection of buried mines, however, will require the use of time-dependent IR signatures caused by diurnal temperature variation of the sun's cycle. Because the thermal conductivities of metal and plastic mines are different from the background soil, temperature contrasts can be seen at the surface during certain times of the day, such as during sun rise and sun set. We propose to use dual-band IR imaging in the 3-5 $\mu \mathrm{m}$ and 8-12 $\mu \mathrm{m}$ ranges; with image fusion, correlation, and other image processing techniques, positive identification of mines with low false alarm rates is possible.

Argonne National Laboratory has used time-resolved IR imaging for nondestructive evaluation of debonds, voids, inclusions and other defects in coated and multilayered materials. The technique has also been successfully used to identify density variation, fiber layer delamination, cracks, fiber-matrix debonding, and other defects in continuous fiber ceramic matrix composites (CFCC). Figure 1 shows a schematic diagram of the infrared imaging system used for thermal diffusivity measurement of CFCC materials. The front surface of the specimen is heated with a photographic flash lamp and the temperature of the back surface is monitored by a infrared camera. A computer with an on-board frame grabber is employed to acquire and digitize IR images from the infrared camera. An in-house developed software extracts the average gray-scale value which represents the specimen temperature and then proceeds to calculate the thermal diffusivity for the entire surface of the specimen. 


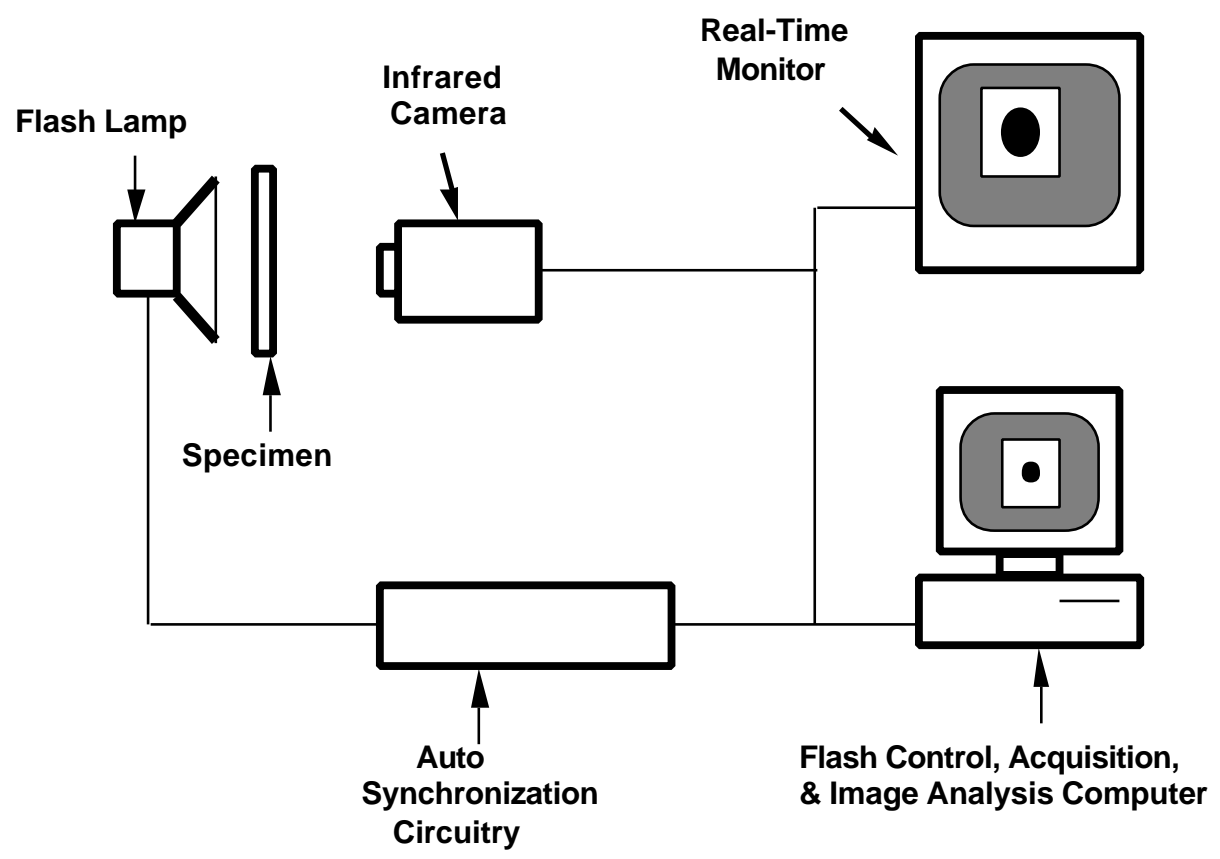

Fig. 4. Dual Band Thermal Imager

\section{Technical Status/Maturity}

Thermal IR imaging as such is a relatively mature technology. Radiometric imaging in the infrared (IR) range has been recognized for remote sensing of Earth from satellites. Earth resources such as vegetation, soil moisture, and snow cover, as well as weather pattern and military targets are routinely imaged with this technology. Argonne National Laboratory has several years of experience with thermal IR imaging for nondestructive evaluation of materials. Over \$1 million of DOE funding has been secured in this area with cost-shared funding from several industries. State of the art IR cameras and systems are available at Argonne, which include inframetrics IR 600 camera, Galileo 256x256 focal plane array camera, image frame grabbers, and image processing software.

\subsection{USE OF MULTISPECTRAL INFRARED IMAGING FOR WIDE AREA SURVEY AND DETECTION OF BURIED LANDMINES}

\section{Technical Contact}

Scott E. Carpenter

Phone: (708)252-5338

FAX: (708)252-5655

E-mail: carpenter@cmt.anl.gov 


\section{Description of Technology}

This project idea addresses the need for a device that can expeditiously survey large areas for landmines, preferably with minimal danger to operators. Newer techniques, such as ground-penetrating radar (GPR), commonly employed in geological work, show promise as effective landmine locators. Unlike conventional mine detectors (metal detectors), GPR would be particularly useful for locating plastic-molded landmines beneath the soil. However, both conventional mine detectors and GPR devices must be methodically moved in a grid-search fashion in order to systematically survey large areas. This approach is time-consuming, costly, and puts the operator(s) at risk. Whether one employs metal detectors, GPR, or mechanical probing, a search based on a priori knowledge from an initial screening of a wide area certainly would be preferable to starting at a random location and conducting an exhaustive search of contiguous square meters of land.

Ideally, this screening sensor would employ passive remote detection. The term "passive" is indicates that the sensor is non-invasive, and the term "remote" is used to indicate that the sensor could detect the target from some distance, which could be several meters to a kilometer. It is also desirable that the sensor be rugged and as lightweight as possible, thereby providing convenient portability and the option of deploying the sensor on a small, low-flying aircraft. This screening device would also need to be a "smart" sensor, which is defined as a sensor that can: (1) interpret multivariate data in real time; (2) can perform data reduction and decision making; (3) can communicate processed results to the user or a data fusion system; and (4) can alter system operation based upon certain results. By definition, this screening sensor would be used to increase the chances of locating landmine positions, thereby providing guidance for search-and-removal activities.

Using infrared (IR) imaging, it is possible to detect small differences between areas where soil has been disturbed and surrounding areas that have not been disturbed. These differences arise because the IR emissivity and reflectance properties of the perturbed soil are different from surrounding areas. Small differences can still be detected for many years after the distress. Therefore, we propose the use of passive remote infrared imaging as a screening tool for detecting buried landmines. The imaging sensor would not directly detect a landmine itself, but it would detect areas where the likelihood of finding buried landmines is great.

The reliability of the proposed screening method can be greatly increased by using multispectral image information. Multispectral images are collected simultaneously for a given scene, but each image contains slightly different information, which is determined by the band of spectral wavelengths comprising each image. Multispectral camera systems spanning visible, near-IR, and mid-IR wavelength spectral regions are commercially available. Obtaining multispectral information is not a problem; the key to the success of this method is simultaneous interpretation of image information from numerous spectral bands. From a practical standpoint this should be done in real-time with little or no intervention by the user. As stated above, the need is for a smart sensor.

\section{Technical Status/Maturity}

Funded Research - DOE Office Sponsored

\section{Equipment Availability}

Computational facilities and software algorithms for processing multispectral image data are available; the proposed Daedalus AMS system, or a similar multispectral camera, would have to be rented or purchased.

The design of automated monitoring algorithms for creating smart chemical sensors is the focus of an ongoing project at ANL, which is funded by the Department of Energy. As part of this project, we have 
recently developed novel algorithms for automated recognition of ships in multispectral images, regardless of the orientation and size of the target objects. These algorithms utilize information from multiple spectral bands simultaneously to produce clutter rejection results that can not be obtained from a single-band image.

This preprocessing procedure is then combined with a unique artificial neural network procedure that can spawn new classes dynamically, thereby providing a way to identify new types of targets, as well as anomalous observations. The multispectral data employed in this work were collected with a Daedalus Airborne Multispectral Scanner (AMS) (Daedalus Enterprises, Inc., Ann Arbor, MI). The Daedalus AMS consists of a dual-element, thermal infrared detector and an 8-channel visible/near-infrared spectrometer. There are a total of 10 user-selectable bands covering the spectral range from $420 \mathrm{~nm}$ to $14 \mathrm{~mm}$; up to six of these bands may be chosen for simultaneous data collection. Of the ten bands, the four near-infrared and mid-infrared spectral bands have provided the most valuable information for ship recognition. It is believed that these algorithms could be modified and adapted for recognition of disturbed soil areas. This would require the use of representative data sets collected over a "dummy" minefield, which has soil characteristics similar to those found in Bosnia.

\title{
1.9 PASSIVE MILLIMETER-WAVE IMAGING
}

\section{Technical Contacts}

\author{
N. Gopalsami and A. C. Raptis \\ Energy Technology Division \\ Argonne National Laboratory \\ Phone: (630) 252-5925 \\ Fax: (630) 252-3250 \\ E-mail: gopalsami@anl.gov
}

\section{Description of Technology}

Passive millimeter-wave (mm-wave) imaging makes use of thermal emissions (blackbody radiation) of objects at millimetric wavelengths (1-10 mm). Radiometric imaging in the visible and infrared (IR) range has been recognized for remote sensing of Earth from satellites. Earth resources such as vegetation, soil moisture, and snow cover, as well as weather pattern and military targets are routinely imaged with these technologies.

Unlike visible and IR systems, millimeter waves can penetrate through cloud, smoke, dust, dry leaves and thin layer of dry soil. In addition, the mm wave technique offers different thermal contrasts of objects, thereby providing different view of the target scene. Thus, mm-wave imaging is a valuable addition to the visible and IR systems. The objective of this proposal is to build and test vehicle and/or helicopter-mounted mm-wave radiometric imaging systems for fast detection and screening of wide areas laden with antipersonnel mines. Metallic mines generally offer good thermal contrast from the soil background and are easily detectable. Plastic mines offer less contrast than metal mines but are still detectable. Thermal contrast of these targets will depend on the fill factor, the amount of cloud cover, and the depth and water content of soil cover. The choice of frequency is important; lower frequencies allow better ground penetration but will suffer from poor spatial resolution and small fill factor. The latter drawbacks can be overcome by using large antenna apertures and short ranges. Feasibility of the technique will be first assessed by developing a performance model that will estimate the detection depths of buried mines of different types and sizes under different soil environments cluttered with false targets. The model will be used also to identify ranges of frequency, antenna aperture, range, look angle, and polarization effects. 
Figure 5 is a 94-GHz mm-wave radiometer system designed and built by Argonne and Millitech Corporation for thermal signatures mapping and effluent chemical detection for arms control application. Using a local oscillator at $85.3 \mathrm{GHz}$, the RF radiation is down converted to four $500 \mathrm{MHz}$ wide IF channels. A diode detector (square-law type) converts the RF power in each channel into a voltage signal, which is further amplified and filtered by a video amplifier. The output signal of each channel is proportional to the radiation power in the corresponding RF channel, which in turn is proportional to the brightness temperature (equivalent temperature with reference to a blackbody) of the scene in the field of view of the antenna.

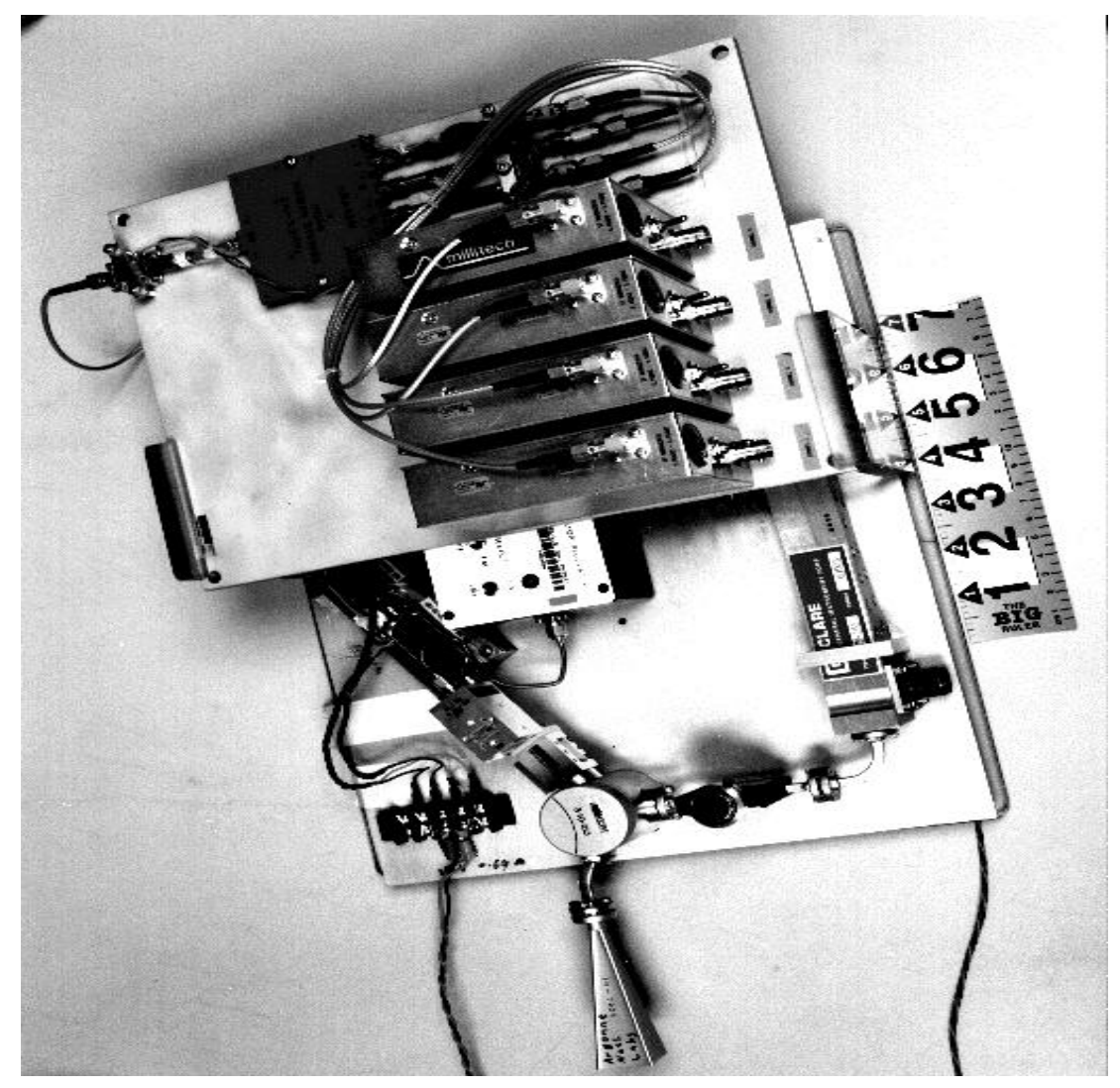

Fig. 5. Four channel radiometer.

\section{Technical Status/Maturity}

Passive mm-wave imaging is relatively a mature technology in low frequency ranges (up to $94 \mathrm{GHz}$ ). Modern mm-wave radiometers can measure temperatures to a resolution of less than $1 \mathrm{~K}$ with $10 \mathrm{~ms}$ integration time. Argonne National Laboratory has several years of experience with $\mathrm{mm}$-wave radiometric technique for both thermal mapping and gaseous effluent detection. Over $\$ 2$ million of DOE funding has been secured in the passive and active mm-wave area over a 6-year period. Laboratory demonstration of the multichannel system has been shown for chemical detection. Radiometers at $94 \mathrm{GHz}$ and $300 \mathrm{GHz}$ are available, together with imaging scanning systems at Argonne. Active mm system is in the field testing stage. Argonne has also developed radiometric modeling capability to predict brightness temperatures and thermal contrasts of objects under realistic scenarios. 


\subsection{ANALYSIS OF NUCLEAR MINE-DETECTION TECHNOLOGIES}

\section{Technical Contact}

Dr. Bradley J. Micklich

Technology Development Division

Argonne National Laboratory

Phone: (630) 252-4849

Fax: (630) 252-1885

E-mail: bjmicklich@anl.gov

\section{Description of Technology}

Argonne National Laboratory has a long history in the development of nuclear technologies for a variety of research and industrial applications. Current ANL research programs center on evaluating a wide range of nuclear and photon-based techniques for detection of explosives and narcotics. For the Federal Aviation Administration, ANL has conducted research on fast-neutron transmission spectroscopy (FNTS) for explosive and narcotics detection in airline luggage. For the Counterdrug Technology Assessment Center, ANL has investigated a variety of non-intrusive inspection techniques for examining empty and loaded cargo containers. The techniques under investigation have included pulsed fast-neutron analysis, high-energy and low-energy x-ray systems, and inspection using radioisotope gamma sources. Argonne is also evaluating advanced high-intensity monoenergetic gamma sources for non-intrusive inspection, and is involved in neutron source development for applications such as neutron radiography, boron neutron capture therapy, and material tagging. These programs make use of ANL's expertise in nuclear data, nuclear experimentation, and radiation transport modeling. A key advantage of these modeling efforts is the ability to characterize system performance without major capital outlays for hardware.

\section{Technical Status/Maturity}

The use of analytic and computer models to describe and analyze nuclear systems is well established. Argonne's experience in nuclear system analysis is a direct result of ANL's history of reactor and accelerator experimentation. The Technology Development Division at Argonne has several current programs in the analysis of nuclear and photon-based interrogation systems, supporting approximately three FTE. These programs have included work on a large range of concepts, and include basic nuclear physics, engineering system studies, assessment programs for candidate systems, data acquisition and analysis, and data imaging. A full range of the necessary analysis tools are in place and in routine use.

\subsection{ACOUSTIC METHODS FOR LAND MINE DETECTION}

\section{Technical Contacts}

H. T. Chien, S. H. Sheen, and A. C. Raptis

Energy Technology Division

Argonne National Laboratory

Phone: (630) 252-4901

Fax: (630) 252-3250

E-mail: ht_chien@qmgate.anl.gov 


\section{Description of Technology}

Low frequency acoustic waves have been used for underground oil exploration. Because of long wavelength, the acoustic waves can propagate a long distance in a porous medium. When a high-intensity longitudinal wave impinges on the soil, in addition to the longitudinal wave, surface waves will also be generated and propagate away from the source. If acoustic pulses are used, echoes can be detected when the pulses are reflected by an object of different acoustic impedance from the soil. Hence, use of array transducers (e.g. phase array) and pulse operation, one can produce an image of the ground soil within the wave propagation range. The image represents an echo map that shows the underground objects such as rocks, roots, and mines. To identify the mines, it requires additional signal processing techniques such as pattern recognition and image reconstruction. Images may be produced from both the longitudinal and the surface waves.

The approach that may be used for the actual field implementation is shown in Fig. 6. An array of acoustic waveguides is attached to a robotic arm whose position is controlled by a remotely controlled vehicle. An acoustic transducer is embedded in every waveguide to provide the acoustic pulses and to act as a receiver for echo detection. Reflected echoes and induced acoustics will be analyzed for positive identification of the mine and distinguishing plastic from metal. To detect underground mines, imaging or tomography techniques are required. This may be accomplished by pulsing the transducers sequentially and analyzing the returned echoes.

The acoustic mine-detection system will be constructed at a relatively low cost and the waveguide assembly can be easily replaced if necessary. Thus, the mine detection will not be interrupted in the case of surfacemine explosion triggered by the waveguide.

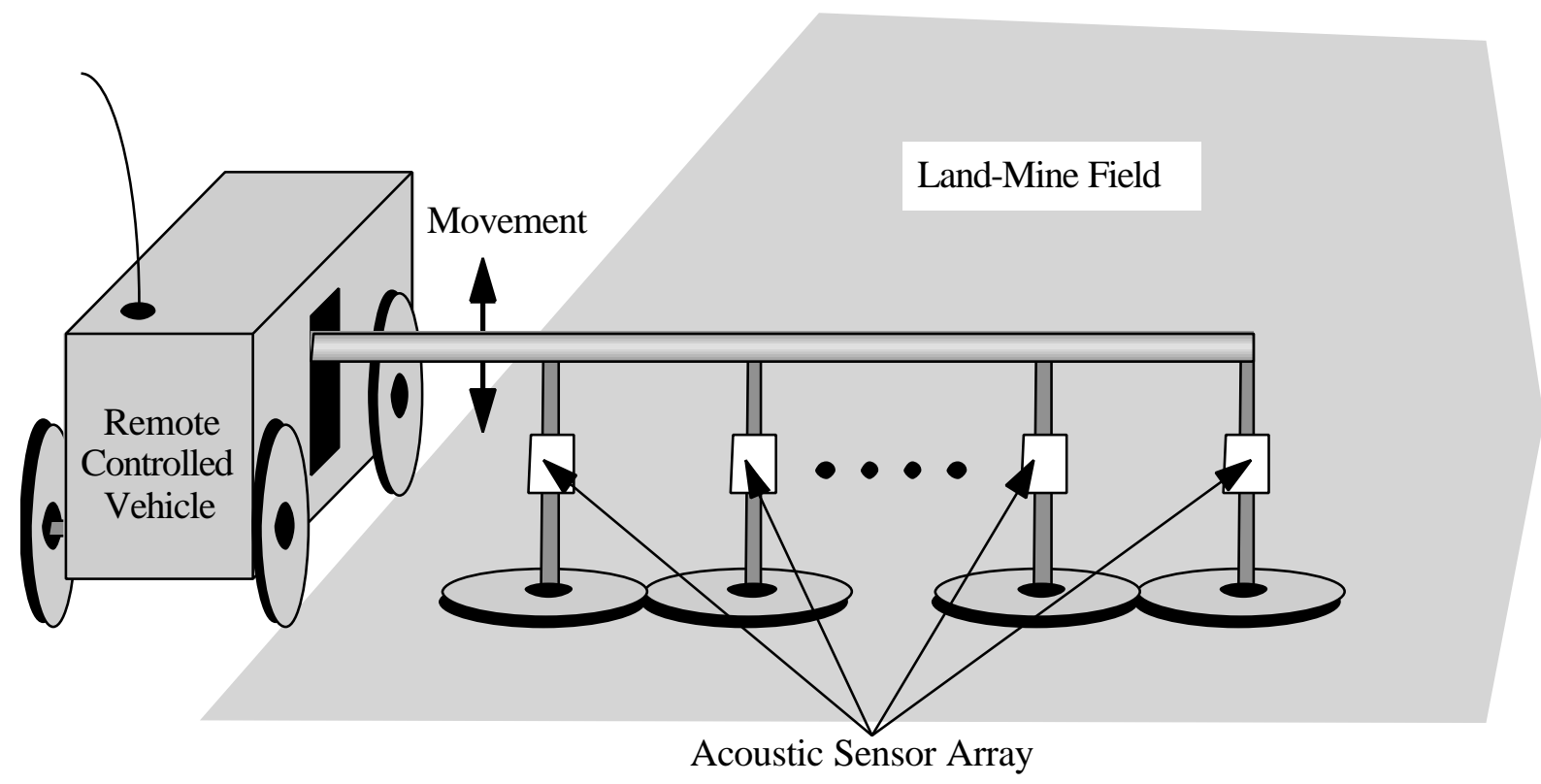

Fig. 6. A conceptual design of a robotic acoustic land-mine detection system. 


\title{
Technical Status/Maturity
}

Argonne National Laboratory has been developing ultrasonic imaging techniques for detecting internal and surface defects of various industrial materials and components. A tomographic technique is being developed for real-time imaging of multiphase flows. Both areas of technology can be directly applied to land-mine detection. Low frequency transducers have been developed using piezoelectric thin films (e.g. PVDF films). A multiple layer PVDF transducer was constructed and tested for monitoring vehicle motion. This PVDF transducer technology can be applied to the transducer assembly shown in Fig. 6.

\subsection{SAW AND FPW CHEMICAL SENSOR ARRAYS FOR AIR AND GROUND-WATER MONITORING AT LAND-MINE SITES}

\section{Technical Contacts}

\author{
S. H. Sheen, H. T. Chien, and A. C. Raptis \\ Energy Technology Division \\ Argonne National Laboratory \\ Phone: (708) 252-7502 \\ Fax: (708) 252-3250 \\ E-mail: sh_sheen@qmgate.anl.gov
}

\section{Description of Technology}

Two micro sensor technologies, surface acoustic wave (SAW) and flexural plate wave (FPW) chemical sensors, have been recently developed for gas emission monitoring. Both sensors show high sensitivity of ppm detection level to most emission gases that are of environmental concern, such as $\mathrm{No}_{\mathrm{x}}, \mathrm{CO}$ and hydrocarbons. Both technologies rely on use of a surface coating that is sensitive to the emission gas but differ in their detection mechanism. A SAW sensor measures the frequency shift of the surface wave propagating on a piezoelectric substrate, whiles a FPW sensor measures the plate wave velocity changes. Fig. 7 shows the basic designs of both sensors.

A SAW device uses a delay line configuration that consists of two sets of lithographically patterned interdigital electrodes (IDTs) deposited onto the surface of an optically smooth piezoelectric substrate. One set of the IDTs generates a high frequency Rayleigh wave that propagates along the device surface and is detected by the second set of the IDTs. When the SAW device is used as the feedback element in an oscillator circuit, the frequency of the circuit is linearly related to the surface wave velocity that is strongly perturbed by surface mass loading. A SAW chemical sensor requires a chemical sensitive coating applied to the substrate surface between the two IDTs. When vapors sorb into the coating, there is an incremental change in the coating density. Variations in the density, viscosity, and elastic properties of the coating will affect the phase, amplitude, and velocity of the surface acoustic wave. For SAW chemical sensors, it is the density or mass loading change that makes the SAW chemical detection possible.

A FPW chemical sensor has a design similar to a SAW chemical sensor. The only differences between the two are (1) FPW devices operating in low frequencies, a few $\mathrm{MHz}$ as opposed to hundreds $\mathrm{MHz}$ for SAW sensors, and (2) the interdigital transducers (IDTs) being embedded on the opposite side of the FPW sensing material, thus the sensor can be applied to liquids because the IDT electrodes are insulated from the liquid. The basic elements of a FPW chemical sensor consist of a composite plate of low-stress materials (e.g. silicon nitride, zinc oxide and aluminum) fabricated on a silicon substrate, a pair of IDTs on the $\mathrm{ZnO}$ layer, and a chemical sensitive film bonded to the liquid-side material (silicon nitride membrane). The IDTs launch 
and receive the plate waves (e.g. the zeroth-order antisymmetric Lamb waves) and together with an amplifier form a feedback oscillator whose output frequency provides a measure of the mass per unit area of the membrane including the chemically sensitive film. The sensitivity of a FPW device is inversely proportional to the mass per unit area of the chemically sensitive layer of the device and can be increased independent of the wavelength by using a small plate thickness.

For land-mine detection, SAW chemical sensors will be used for air quality monitoring and the FPW sensors will be used for ground-water monitoring. The SAW sensors will focus on detection of organic compounds, particularly nitro-aromatic molecules. The FPW sensors will apply to detection of inorganic salts dissolved in ground water with emphasis on potassium-ion increase after a rain fall. The SAW sensing system will include an array-sensor design that will use different surface coatings and pattern recognition techniques. To locate the mines, multiple sensing stations will be used to determine the chemical concentration profile from which the mine locations may be established.

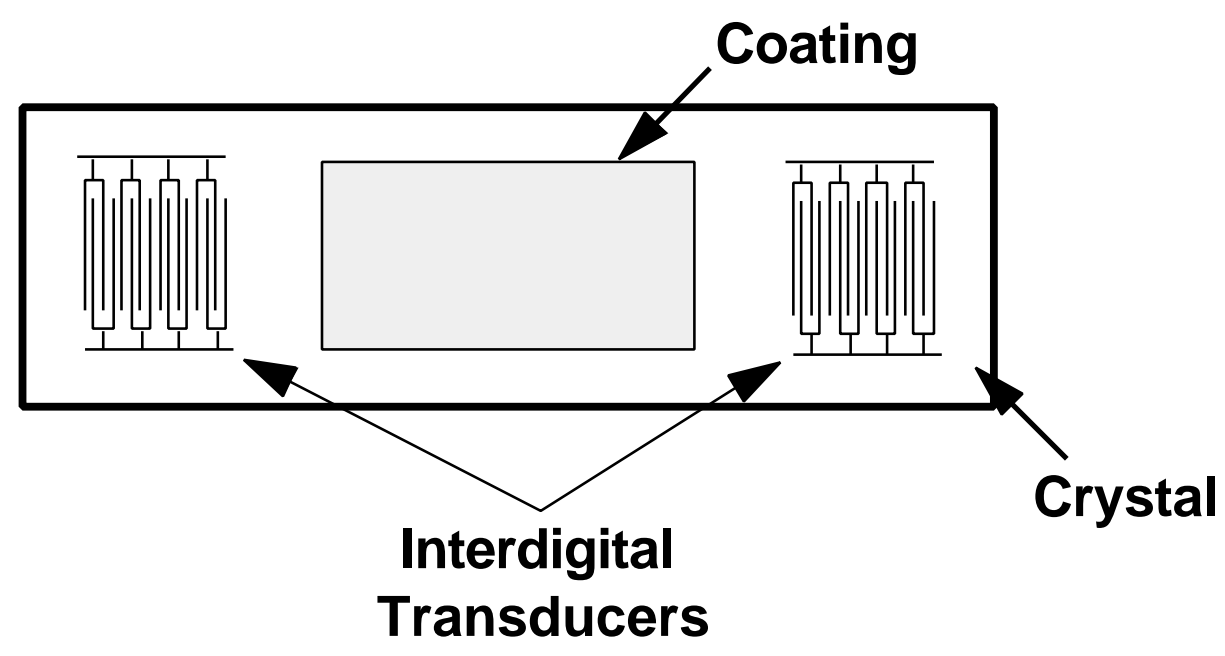

Fig. 7A. The basic configuration of a surface-acoustic-wave chemical sensor.

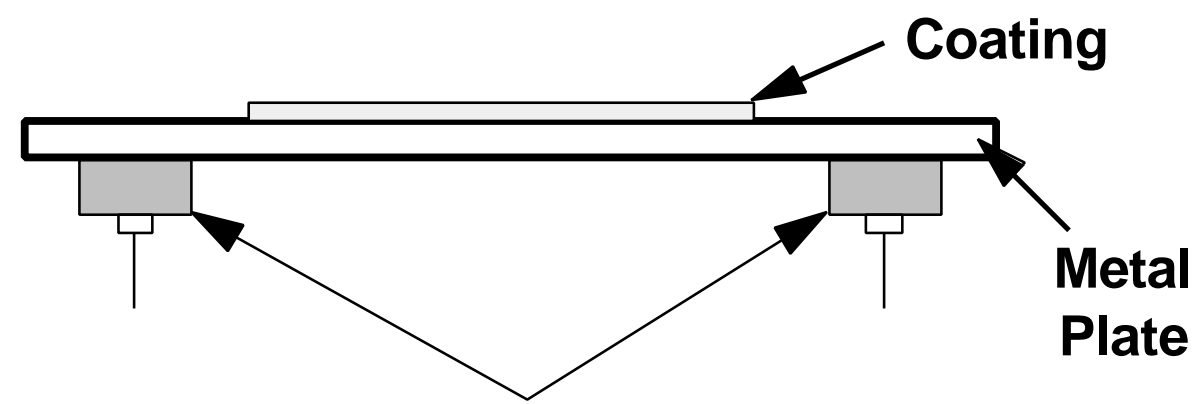

Transducers

Fig. 7B. The basic design of a flexural-plate-wave chemical sensor. 


\section{Technical Status/Maturity}

Argonne National Laboratory is currently developing both SAW and FPW chemical sensors for auto emission monitoring. Several surface coatings are being examined for detecting hydrocarbons. Multiple sensor array is also being designed and tested. For liquid-phase applications, the FPW technology is examined. A static electrical field is applied to the FPW cell for detection of ions in a solution. Both sensor systems can be fabricated at low cost and in rugged design for field application.

\subsection{SWITCHABLE RADIOACTIVE NEUTRON SOURCE}

\section{Technical Contact}

Dr. Alexander DeVolpi

Phone: (630) 252-4598

Fax: (630) 252-3078

E-mail: devolpi@td.anl.gov

Description of Technology

A fieldable nuclear technique that could assist in mine-detection is the switchable radioactive neutron source (SRNS) matched to a linear hodoscope array of neutron and gamma detectors or to any other detection system requiring a neutron source. The SRNS is a portable radioactive neutron source that can be switched on and off by simple mechanical action. It does not require the electric power or ancillary equipment needed for accelerator sources. The SRNS can produce penetrating neutrons of several $\mathrm{MeV}$, as well as gamma rays. Neutron inelastic scattering and capture would provide ratios of elements $\mathrm{H}, \mathrm{C}, \mathrm{O}$, and $\mathrm{N}$ to reasonable depths. The most probable neutron interaction would be elastic scattering, especially in the light elements (that are more characteristic of explosives than background). In addition, gamma rays produced by the source would interact by Compton scattering, photoelectric absorption, and pair-production.

A primary advantage of the SRNS is that neutrons can be switched on and off without complex electronics. Remote switching control can be effected by either mechanical linkage or battery-powered solenoid. Neutrons from the SRNS can be a distributed across a wide path, rather than being limited to a point source. As a result of these design features, the SRNS offers reduced radiation hazard and more efficient use of neutrons for penetrating interrogation. Hodoscope systems have been developed and applied for challenging detection problems that involve radiation sources in nuclear-reactor experiments and adapted to arms control and nonproliferation. The SRNS system combined with neutron/gamma hodoscope could operate synergistically with non-nuclear techniques to make time- and cost-effective searches that would result in high probability detection of anti-personnel and anti-tank mines, whether made with plastic or metal shells.

\section{Technical Status}

A laboratory demonstration of switchable source proof-of-principle under DOE was completed. Design concepts exist for remotely controlled switchable radioactive sources that would have neutron intensities and spectra appropriate for mine detection. 


\subsection{MICROWAVE IMAGING WITH SELECTIVE CHEMICAL OR BIOLOGICAL ADSORPTION TECHNIQUE}

\section{Technical Contacts}

N. Gopalsami, S. Bahtiari, S. H. Sheen, and A. C. Raptis

Argonne National Laboratory

Phone: (630) 252-5925

Fax: (630) 252-3250

E-mail: gopalsami@anl.gov

Thomas Lavin, M.D.

Gene Inc.

Phone: (908) 756-4006

Fax: (908) 755-7266

E-mail: tlavin@genesis.com

\section{Description of Technology}

At present, detection of plastic housed land mines in shallow subsurface locations is difficult if not impossible because of the non-reflective nature of plastic housings in land mines. Further the trade off between lower frequencies required for ground penetration and higher frequencies required for good spatial imaging necessitates the search for a soil penetrating agent which can selectively enhance microwave imaging of buried plastics versus natural occurring soil constituents.

Conversion of the surface of the hydrophobic plastic housing to a hydrophilic one would render the land mines reflective to microwave energies. We propose to develop a method to accomplish this in a fashion which would allow selective adsorption to the plastic housing relative to surrounding soils. It is envisioned that the affected areas could be drenched with detection fluid, then with water to disperse non-bound material. The coating-adsorption process would be biodegradable in the field. Several techniques are envisioned to accomplish this in situ:

1. Use of amipathic surfactants which create a hydrophilic exterior surrounding a hydrophobic region next to the plastic. Such surfactants would require polyester backbone to allow biodegradation.

2. Use of ampipathic biodegradable polymer coatings such as Aquamer, polyvinyl butryal or polyvinylalchohol in an ethanol/water solvent to coat the plastic housing in situ.

3. Use of biological surfactants such as lecithin or other phospholipids which are readily obtainable from commercial sources or can be tailored to have a specific hydrophobicity.

4. Seeding the ground with polycyclic aromatic hyrdrocarbon metabolizing bacteria and phosphate and nitrate containing fertilizer which degrade, at least to a small degree, the plastic housings and produce a water and bacteria containing coating around the mine. Such bacteria are currently used in oil spill bioremediation, vehicle oil clean up in automotive parts manufacturing. Because the type of plastic in the land mines is unknown at this time, the degree of metabolism which may take place cannot be predicted until field trials are undertaken. It is expected that the shallow placement of most mines will allow aerobic conditions in the soil and permit bacterial growth. Certain other species of bacteria which may be more effective have been used by Mitsubishi and MIT. 


\section{Technical Status/Maturity}

Microwave noncontact probing techniques offer high resolution and sensitivity for examination of a wide range of dielectric media. Due to versatility of such systems, their range of application extends from nondestructive evaluation of advanced dielectric composite materials to medical imaging area. Advancements in the microwave solid-state technology and real-time computer based imaging have further increased the applicability of microwave sensors for real-time insitu applications. Feasibility of high-resolution microwave imaging using fixed frequency coherent sources have been demonstrated to some extent in the past. Research on new wavefront processing algorithms is currently being pursued to enhance real-time image reconstruction for detection of buried objects. With the component dimensions being in the order of operating wavelength at microwave frequencies (cm range), compact microwave imaging systems would inherently offer great potential for such applications. Further, with the antenna beamwidth being inversely proportional to the operating frequency, higher microwave frequencies (i.e. shorter wavelengths) inherently allow for finer resolution probing given that the dielectric losses inside the material medium allow for detection of backscattered signal. The penetration of microwave radiation inside most nonconducting, dry, solid media is suitable for detection of buried objects at multiple wavelengths beneath the surface which is also well within the detection range of solid state receivers. The upper limit on the usable frequency is dictated by material complex dielectric properties and the state of technology. Quasi far-field lens antennas using focused beams may be used for fine resolution measurements to be made at any distance from the material surface under test. Such sensors are ideally suited for insitu measurements in harsh environments.

Some representative c-scan images have been made with ANL's benchtop millimeter wave reflection/transmission sensor for some dielectric composite samples with artificially embedded defects. The samples consisted of plates of Kevlar-fiber-reinforced-epoxy composite which contained subsurface voids and disbonds. The low-power W-band system which incorporates Gaussian optic lens antennas allows for high resolution inspection of materials with minimal diffraction losses. With minor modification of the system, such techniques as synthetic aperture imaging may be readily implemented for wide area monitoring applications. 


\title{
2. BROOKHAVEN NATIONAL LABORATORY
}

\subsection{THERMAL NEUTRON CODED APERTURE DETECTION}

\author{
Technical Contact \\ Leon Forman/Peter Vanier \\ Phone: (516) 344-5852/3535 \\ Fax: (516) 344-7533 \\ E-mail: leon@bnl.gov/vanier@bnl.gov

\section{Description of Technology}

BNL has developed a coded aperture thermal neutron camera that has successfully detected neutron sources at a range of over 200 feet. This camera has the potential for finding land mines when fast neutrons from an accelerator illuminate the ground because there will be a significant thermal neutron production increase in regions containing hydrogen, such as is present in high explosive. The mine should appear as a bright pixel in the thermal neutron image. Experiments have been conducted which demonstrate the concept, however, the envelope of performance must be established to compare this approach with competing technologies.

\section{Technical Status}

A thermal neutron coded aperture camera prototype has been constructed and tested at BNL for the purpose of detecting thermal neutron sources at long range. The detector is a crossed wire He-3 chamber (designed for neutron diffraction studies) with limited availability. A chamber designed for land mine detection should be constructed as one of the milestones of this project.

\section{Funded Research}

DOE - FY97 status not known

\section{Equipment Availability}

Adequate for envelope of performance evaluation.

\subsection{DETECTION OF LAND MINES USING ELASTIC WAVE SCATTERING (EWS)}

\section{Technical Contact}

Nick Simos

DAT-SSN Division

Phone: (516) 344-7229

Fax: (516) 344-7650

E-mail: simos@bnl.gov 


\section{Description of Technology}

This proposed idea of mine detection is built on the principle of resonance scattering of elastic waves. In particular, the scattering of such waves is induced by elastic/solid objects imbedded

in an elastic or visco-elastic medium such as soil.

Since no single method is expected to be suitable for all types of mines, pattern of field arrangement and terrain, the proposed method is expected to work best in vehicular safety against land mines where the terrain involved is either roads or relatively smooth surfaces. Wet terrain conditions will not incapacitate the method.

For a finite object imbedded in a medium of different properties, elastic waves will propagate in the matrix medium and subsequently interact with the object in the form of scattering. It is expected for the object to sustain vibrations as a result of the scattering. Since the object (such as a mine) has characteristic eigen frequencies, its vibration at these frequencies will be excited if they coincide with the frequency of the incident wave. It thus becomes intuitively clear that if such frequencies are known in advance (or at least approximately), the induced excitation of the medium in the form of elastic waves can be narrowed in the vicinity of these frequencies. It should thus be anticipated that the clutter, resulting from scattering of all kinds of buried objects, is reduced. The task is to mathematically or computationally identify them for the different types of buried mines one anticipates to find buried in the field (steel, plastic, shape and size). Further, the variability in the soil is also expected to shift the frequencies and thus it needs to be addressed.

While technology in this area has been advancing and is being put to good use, tuning of the technology is required as well as coupling with advancements in signal processing. Recognition and location of buried mines will be obtained from multiple geophones arranged in a pattern that will prove to be effective and from processing/correlation of the multiple signals.

\section{Technical Status}

The technology of resonance scattering of elastic waves has been developed and put to good use in a number of technical applications. Specifically, in the areas of seismology, nondestructive material testing, mineral and oil exploration as well as medical applications using ultrasonics. Using the principles of the theory, identification of fluids in cavities has been made possible. Thus, given the advancement in signal processing, this technology can be modified and geared toward mine detection. BNL and in particular DAT/SSN has been directly involved in actual recording, processing and identification of ground motions emanating from different sources within the laboratory grounds.

\section{Funded Research}

Internal/Laboratory

\section{Equipment Availability}

Equipment to be used is available through:

(a) outside BNL consultants who have applied various technologies in field explorations including seismic refraction/reflection and,

(b) the Department of Advanced Technology (BNL) designed for ground vibration survey work (accelerometers, geophones, FFT analyzer, power unit and filters). 


\section{Applications/Testing}

Testing of the developed technology (geared for mine detection) will be performed on BNL grounds.

\subsection{RF DETECTION AND NEUTRALIZATION OF LAND MINES}

\section{Technical Contact}

George Allanson Greene

Brookhaven National Laboratory

Building 820M

Upton, New York 11973

Phone: (516) 344-2296

Fax: (516) 344-3526

E-mail: GREENE1@BNL.GOV

\section{Background}

The threat posed by land mine ordinance cannot be neutralized by a single technique or technology due to the complexity of the various devices and the extent of their deterioration due to exposure. Many mines that have been deployed lie abandoned in or on the ground and their mechanisms have deteriorated. As a result, triggering and firing mechanisms have become defective and unstable. Neutralization of such devices in this condition by a hands-on approach is very dangerous. Even primitive anti-tank mines may have so little metal that they cannot be detected and discriminated from battlefield shrapnel and other debris by the use of handheld metal detectors. Therefore, techniques for neutralization must be robust and exploit a generic attribute of these devices which will be effective at neutralizing all or most of such devices.

\section{Description of Technology}

Most land mines utilize electric blasting caps to initiate the primary explosive. Blasting caps and other electric detonators can be stimulated to explode by the application of a direct or alternating electric current along their lead wires; this stimulus can be supplied by a battery or hand-held firing device. Electric blasting caps are so vulnerable to electric pickup that standard operating procedures in the military require that blasting caps be transported separately from explosive, and that the caps be shielded, if possible, from radio transmitters. It is proposed to develop a RF transmitter system capable of inducing electric currents in the detonators of field deployed land mines to exploit the basic mechanism of operation of most land mines, electrical transmitter design and selection of operating parameters. Field demonstration of in-situ neutralization of mines by either detonation of the devices (detonator and mine) or destruction of the wires in the detonators itself by induced electric currents will be conducted to evaluate operating parameters and transmitter design. The device thus designed should be portable and field tunable, capable of broadcasting a variable frequency, variable intensity, directional RF waveform, with the capability of amplitude and frequency modulation. Simulated field demonstration will be conducted to demonstrate proof-of-principle, efficiency and reliability, and feasibility of deployment.

\section{Technical Status/Maturity}

The conceptual ideas being proposed for this technological approach to land mine neutralization is innovative in so far as it builds upon proven fundamental concepts of electrical science and shows promise of rapid demonstration and deployment. The basic idea is to supply a very high voltage excitation signal to a passive 
spark generator. At the frequency of the supply signal, a high voltage spark will bridge a tunable gap, which in turn will be fed to a RF generator coil in order to produce a 60 cycle, high intensity, RF field, composed primarily of noise signals of variable frequency and intensity; with the use of dimple directional transmission antennae hardware, the RF "noise" will be directed at the target ground area in order to induce electric currents in the detonators in the affected region and either detonate the explosive devices or incapacitate their detonators. The RF transmitter device should be durable and portable, and able to be powered by an ordinary electric generator or by the power pack of a military vehicle, such as a battle tank. The transmitter should be constructed from readily available materials, be modular to facilitate interchange of parts, and inexpensive and easily replaceable.

At the present time, no directly related projects are funded at the Brookhaven National Laboratory (BNL). However, experience gained in other projects which involve high voltage RF transmission can be leveraged to enable BNL to quickly and efficiently make immediate progress toward laboratory tests and prototype development demonstration. A significant amount of capital equipment is available that can be used for the purposes of these prototype evaluations and field demonstrations, this impacting favorably on schedule and reducing short term costs. BNL has a dedicated Instrumentation Division and numerous accelerator projects involved in RF technology whose collaboration can be enlisted on a service basis. Laboratory and field facilities are available on site for use, some of which have been previously certified for explosive activities. In addition, several US Army arsenals are located nearby which could serve as a test range at the appropriate time.

BNL is prepared to begin work on this concept as soon as funds are committed to the project. A funding level sufficient to support the activities of 1.5 scientific and 1.0 technical personnel is projected for the first fiscal year at a budget of $\$ 500 \mathrm{~K}$.

\subsection{NEUTRON SOURCE}

\section{Technical Contact}

\section{Todosow}

Phone: (516) 344-2445

Fax: (516) 344-7650

E-mail: Todosowm@bnl.gov

\section{Description of Technology}

The neutron source technology being proposed in this concept is based on the interaction of medium energy protons (MeV range) with targets composed of light nuclei. The particular reaction of interest in this case is $\mathrm{Li} 7(\mathrm{p}, \mathrm{n}) \mathrm{Be}$, which has a threshold at approximately $1.9 \mathrm{MeV}$; and produces neutrons in the $\mathrm{keV}$ range. Protons of interest for this application can be produced by a Radio Frequency Quadruple (FRQ). These accelerators are relatively short $(\sim 2 \mathrm{~m})$ and can thus fit on a vehicle. In addition, sufficiently intense proton beams can be accelerated to produce neutron sources of high enough intensity to be of interest. The source neutrons will be moderated to the appropriate energy to either excite capture levels in the target of interest, or be moderated in the target and be back scattered. In either case, the returning signal will be measured in an appropriate counter. Variations in the proton energy result in variations in the neutron energy; thus a variety of neutron induced reactions can be evaluated rapidly.

It is proposed to design a neutron source system (RFQ/Target/Moderator) which would fit onto the back of a vehicle, and be transported into the field. A drift tube/magnet system would transport the proton beam to the 
target/moderator at the end of a boom extending off the end of the vehicle. The neutron beam would be directed to the spot of interest, and detectors on the boom would determine the response. 


\title{
3. IDAHO NATIONAL ENGINEERING LABORATORY
}

\subsection{EXPLOSIVES DETECTION USING ION MOBILITY SPECTROMETRY}

\section{Technical Contacts}

David A. Atkinson, Ph.D.

(208) 526-9745; bigdog@inel.gov

Robert G. Ewing, Ph.D.

(208) 526-9745; rewing@inel.gov

David L. Miller, Ph.D. (Research Manager)

(208) 526-9052; bsh@inel.gov

\section{Organizations}

Chemical Materials and Processes

Sensor Products

\begin{abstract}
The INEEL has an extensive program in explosives detection, covering areas such as aviation security, mine detection by vapor signature, improvised explosive device (IED) identification, post-blast analysis, and environmental characterization of explosives contaminated soils and water. Although numerous analytical techniques are used routinely, ion mobility spectrometry (IMS) has been demonstrated as the most effective tool in explosives detection and identification. The INEEL has a very comprehensive ion mobility spectrometry research program, with studies ranging from basic instrument design to method development and implementation for highly specific applications.
\end{abstract}

\section{Stage of Development}

The ion mobility spectrometry work at the INEEL covers the whole range of technology development categories. Work is done using and evaluating commercially available instrumentation on one extreme; on the other end of the spectrum, basic research into instrument design and fundamental gas phase ion chemistry is done to support programs.

\section{Intellectual Property Status}

A number of patent applications are currently being prepared from the results of recent research. Two specific patents, one involving a novel non-radioactive ionization source and the other on the manipulation of gas phase reagent chemistry for enhanced explosive residue detection have been submitted.

\section{Competitive Advantage}

Ion mobility spectrometry provides a portable, sensitive, rugged, near-real-time trace organic analysis technique that can be used to provide low cost analytical measurements in the field. The research in ion mobility spectrometry at the INEEL is at the forefront of the technology development and is pushing the 
technique into new application areas, such as the detection of land mines by vapor signature and qualitative analysis of explosives in soils.

\section{Collaborative Partners}

Current collaborations exists with both university and industrial partners. Negotiations are ongoing with new industrial partners to further develop and commercialize the technologies in the submitted patents.

\section{Affected Industries}

This technology has broad applications in the environmental field for near-real-time organic analysis in the field. There are also extensive applications in the law enforcement and national security arenas, such as the detection of explosives, drugs, drug precursors, and chemical warfare agents.

\section{Date Prepared}

$11 / 97$

\subsection{SECONDARY ION MASS SPECTROMETRY}

\section{Technical Contacts}

J. E. Delmore, (208) 526-2820

G. S. Groenewold, (208) 526-2803

A. D. Appelhans, (208) 526-0862

J. C. Ingram, (208) 526-0739

D. A. Dahl, (208) 526-9915

Chemical Materials and Processes

Technology Transfer Office

(208) 526-4299

\section{Description of Technology}

The program develops techniques and instrumentation for analysis of electrically nonconducting samples with secondary ion mass spectrometry (SIMS). This includes development of molecular negative ion and neutral beam sputter sources and methods for controlling sample charging. This program has demonstrated that molecules are 10 to 30 times more efficient than similar mass atoms for sputtering molecular ions in SIMS. A new technique, pulsed extraction, has been developed and patented in the last several years that prevents charge build-up on insulating samples such as polymer films, biological specimens, and plant surfaces when using a negative ion beam. Negative ions beams can now be used in place of neutral beams while avoiding the previously crippling sample charging problems. As such, the scope of this program has expanded to emphasize the application of new, large negative ion, molecular beams.

\section{Collaborative Partners}

A collaborative arrangement is currently being pursued with Physical Electronics, Inc. 


\section{Affected Industries}

The agriculture industry, for detection of surface contaminant's. The Department of Defense, for the detection of nerve gases.

\section{Competitive Advantage}

This technology provides quicker, cheaper, and potentially near-real-time analyses for surface contamination.

\section{Stage Development}

Two activities are in the basic research/emerging technologies arena. Three other activities are in technology development.

\section{Intellectual Property Status}

One patent has been issued, one has been accepted for issuance, and an application for a third patent is now being prepared for submittal. R\&D 100 awards have been issued for activities associated with this technology.

\section{Date Prepared}

$9 / 96$

\subsection{DIGFACE CHARACTERIZATION}

The digface characterization project is an integrated demonstration of multiple sensors used as part of a retrieval effort. The characterization technology will allow continuous and continually improving monitoring and characterization of the site being remediated. The digface characterization technique is integrated into the remediation process itself. As retrieval progresses, sensor data interpretation improve by comparing interpreted data images with the retrieved targets.

Geophysical, chemical, and radiological sensors are being deployed by a robotic system. The sensors scan the surface being remediated. As waste retrieval proceeds, the sensors are continuously deployed to characterize the remaining waste. The remediation process proceeds in steps, the characterization data being interpreted online to support the retrieval process.

Three prototype sensors were developed for digface application: a gamma spectrometer, a dielectric permittivity sensor, and a focused electromagnetic induction sensor. Laboratory data for the dielectric permittivity and gamma spectrometer sensors indicate that both are feasible candidates for digface application.

The pilot-scale testing using a soilbox to simulate the digface for automated deployment of sensors on a robotic gantry crane system is complete. The field testing of a prototype digface characterization system used a test trench at the INEL Cold Test Pit. The system's multiple sensors were deployed with a manual nonmetallic gantry designed and constructed to support obtaining dense data sets in a noninterferring environment.

Data were obtained for several commercial sensors (magnetometer, electromagnetic sensor, chemical sensor) deployed over simulated targets in multiple passes as soil layers were progressively removed. The resolution 
capabilities of the sensors have been evaluated and are able to support development of real-time interpretation of targets needed for digface application.

The prototype dielectric permittivity sensor, gamma spectrometer/chemical assay system, test sensors, and gantry crane and Schilling arm were built and the integrated system is now ready for complete full-scale demonstration.

\section{Competitive Advantage}

The digface characterization approach is applicable to any waste site undergoing retrieval. The technology is intended to be adaptable as required for a specific site.

The digface technology reduces environmental, health, and safety risks during cleanup of buried waste sites. Real-time data interpretation during the retrieval process allows for incorporation of appropriate remediation equipment to maintain safety and environmental standards.

Multiple sensors characterize the digface during progressive soil removal. The sensors measure geophysical, radiological, and chemical properties. Development of an automatic deployment capability and development of refined data interpretation techniques support rapid target identification and are powerful features of this technology.

\section{Stage Of Development}

Prototype stage.

\section{Intellectual Property Status}

The successful completion of the demonstration resulted in a proven concept that is ready for technology transfer. Vendors participating in the project supplied the base instrumentation and equipment for the sensors (Geonics Ltd.). Others involved in the project include those involved in waste cleanup and working with environmentally hazardous material (Ecology International), robotic programs (INEL Buried Waste Robotics Program), and mining interests (Colorado School of Mines and U. S. Geological Survey).

Individual sensors as well as combined systems (sensors and robotic arm) have commercial potential in manufacturing, mining, and waste cleanup operations.

Other Department of Energy and Department of Defense sites have been contacted to identify an agency that is planning an actual remedial or removal action for a radiologically and chemically contaminated site. Their collaboration with the INEL will be sought to use the digface technology in the remediation activity as a means of realistically demonstrating the technology.

\subsection{RAPID GEOPHYSICAL SURVEYOR}

\section{Technical Contacts}

Glen S. Carpenter (208) 526-1542

Nick E. Josten, (208) 526-7691, nj2@inel.gov

Lyle G. Roybal

Environmental Restoration Technology Department 


\title{
Technology Transfer Office
}

(208) 526-4299

\begin{abstract}
The Rapid Geophysical Surveyor (RGS) is a handpushed, nonferrous vehicle that carries multiple cesium total field magnetometers, a data logger, and data storage hardware and software. The unit can be easily adapted to accommodate different magnet sensors. Magnetic data are collected automatically and stored at userspecified intervals as close as $2 \mathrm{in}$. apart as the unit is pushed along survey profile lines. The resulting database has very high resolution and can identify individual metallic objects, object orientation, shape, and depth of burial.

The RGS was successfully demonstrated at the INEL. Cold Test Pit and then used to scan buried waste at Pits 7 and 9 at the Radioactive Waste Management Complex and at Los Alamos National Laboratory. Straightforward interpretation principles were applied to the magnetic data, which provided reliable detection of all the isolated ferrous objects in the Cold Test Pit Characterization Cell, a general depiction of the Retrieval Cell, and an accurate definition of the overall waste pit. The RGS collected spatially denser data sets than previously thought possible, thereby providing a much higher resolution picture of the buried waste pit. Other geophysical instruments might be incorporated on this platform such as gamma detectors, chemical detectors, and an electromagnetic system. The surveyor was developed for less than $\$ 200,000$.
\end{abstract}

\section{Collaborative Partners}

Sage Earth Sciences.

\section{Affected Industries}

DOE, DOD, mining, agriculture, manufacturing, waste management, and emergency response.

\section{Competitive Advantage}

This technology can provide a viable geophysical survey of buried waste sites such as the INEL.

Radioactive Waste Management Complex. it provides a critical data set for waste pits, which results in an estimate of the depth of basalt at pit boundaries, depth to overburden/waste interface, or location of waste and original excavation boundaries.

The RGS can be used at any DOE or industrial facilities that have buried hazardous waste or radioactive waste. It can perform high-resolution geophysical magnetic surveys quickly (30-300 times faster) and more economically (20 times cheaper per data point) than hand-held instruments. Quicker and better geophysical surveys can be performed with the RGS at a significantly lower cost.

\section{Stage Of Development}

Commercialized

\section{Intellectual Property Status}

Disclosure of this research to private sector interests via technical journals and presentations at technical 
conferences and demonstrations have initiated interest in the RGS. The inventors have applied for a patent and have created a company, Sage Earth Sciences, Inc., to market the RGS technology. DOE has created an exclusive license to the inventors.

\title{
Date Prepared
}

$9 / 96$

\subsection{VERY-EARLY-TIME ELECTROMAGNETIC SYSTEM}

\section{Technical Contact}

Mary C. Pfeiffer, (208) 526-1893, mpc@inel.gov

Environmental Restoration Technology Department

\section{Technology Transfer Office}

(208) 526-4299

\begin{abstract}
One-dimensional numerical modeling algorithms were developed and tested and are being used in physical analysis of the buried waste problem, interpretation of field data, and systems analysis devoted to instrument design. An optimal system was designed using physical modeling. A prototype transmitter loop has been fabricated. The existing instrumentation and interpretation systems were field tested at the INEL Cold Test Pit. A one-dimensional interpretation of the high frequency sounder data gave very realistic estimates of the thickness, dielectric permittivity, and electrical conductivity properties of the cap covering the Cold Test Pit waste.
\end{abstract}

An upgraded prototype time-domain electromagnetic instrument and the second generation frequency-domain high-frequency sounder were fabricated and are being field tested. The one-dimensional code, with a graphical user interface, will be transferred to industry at the close of the project. These systems will acquire data in a tenth of the time required by the first-generation instruments.

The very early-time electromagnetic (VETEM) system collects and interpret data from the shallow subsurface. This instrument operates in the region of the electromagnetic spectrum between low frequency electromagnetic induction and ground penetrating radar frequencies. This region of operation allows the system to be used at sites where ground penetrating radar has little success. Physical and numeric models being developed, determine instrument design criteria, and test processing and interpretation algorithms. New modeling, interpretation, and imaging algorithms must be developed to account for both diffusion and transmission effects. The instrumentation, modeling, and interpretations are being developed as a system so that the optimal package of hardware and software is achieved.

\section{Collaborative Partners}

U. S. Geological Survey (USGS)

Lawrence Berkeley Lab 


\section{Affected Industries}

DOE, DOD, mining, agriculture, manufacturing, waste management, and emergency response.

\section{Competitive Advantage}

This technology could be applied to characterizing buried wastes at the INEL Radioactive Waste Management Complex and other DOE sites. Specifically, it could contribute to identifying buried objects by shape, orientation, and location.

The resulting system, along with interpretation and imaging software, enhances resolution of the shallow subsurface. Additionally, this system will operate at sites where the physical properties of the soils make high-resolution ground penetrating radar difficult.

Cost savings can be estimated based on sampling and analysis costs of $\$ 300$ a sample. If the use of nonintrusive characterization systems can reduce the intrusive sampling and analysis load by $75 \%$ of the samples, a typical sampling plan requiring 1,000 samples can be limited to 250 at a cost savings of $\$ 225,000$ per site and a great savings in worker exposure.

\section{Intellectual Property Status}

This technology could be applied to the solution of characterizing buried waste at the INEL Radioactive Waste Management Complex and other DOE sites. Specifically, it could contribute to identifying buried objects by shape, orientation, and location.

The resulting system, along with interpretation and imaging software, enhances resolution of the shallow subsurface. Additionally, this system will operate at sites where the physical properties of the soils make high-resolution ground penetrating radar difficult.

Cost savings can be estimated based on sampling and analysis costs of $\$ 300$ a sample. If the use of nonintrusive characterization systems can reduce the intrusive sampling and analysis load by $75 \%$ of the samples, a typical sampling plan requiring 1,000 samples can be limited to 250 at a cost savings of $\$ 225,000$ per site and a great savings in worker exposure.

\section{Date Prepared}

$9 / 96$

\subsection{CRYOGENIC CUTTING SYSTEM}

\section{Technical Contact}

Dennis N. Bingham

Environmental Restoration Technology Department

(208) 526-1376

bnd@inel.gov

\section{Technology Transfer Office Contact}

Paul Grahovac 
(208) 526-3488

pg2@inel.gov

\begin{abstract}
Cryogenic cutting is an adaptation of the highly effective waterjet technique used in cutting a surface with a high pressure abrading substance. It uses high pressure liquid nitrogen and solid carbon dioxide to perform the cutting and abrading. This substitution removes the secondary waste-stream as a cutting media, which is a highly desirable option for most waste management and environmental restoration activities. The cryogenic cutting technology will enhance existing fluid systems to deliver high pressure liquid nitrogen and solid carbon dioxide through a sophisticated nozzle. The system will be evaluated by cutting select materials.
\end{abstract}

The existing cryogenic cutting system underwent extensive upgrades to improve cutting effectiveness, including enhancements to existing fluid systems for the delivery of liquid nitrogen to the nozzle, development of robust control of the nozzle actuation, and evaluation of cutting on select materials.

The nozzle was attached to gantry crane to allow it to be operated in an 18 in. $\mathrm{x} 18 \mathrm{in}$. $\mathrm{x} 18 \mathrm{in}$. volume, allowing sufficient movement to establish cutting rates with various materials. A plywood box was opened in 15 minutes at a scan rate of $0.5 \mathrm{in} . / \mathrm{sec}$. A steel drum was opened by directing the jet around the periphery of the drum. For one revolution, the travel speed was $0.012 \mathrm{in./sec}$. giving a $0.007 \mathrm{in}$. depth of cut. At this rate, eight passes were required to perforate the drum wall which would take approximately seven hours.

\title{
Intellectual Property Status
}

Several companies have expressed interest in collaborating in the development of this technology.

Technology transfer mechanisms will be used to form the desired partnerships. Interested parties represent cutting service companies, high pressure pump companies, control system companies, and cryogenic companies. Cryogenic cutting may have wide applicability to site decommissioning where secondary waste streams are of concern. Foreign license to Cryela Cryogenics B. V. for European applications.

\section{Competitive Advantage}

Large objects unearthed during buried waste retrieval operations will require size reduction prior to treatment. Current methods for size reduction include shearing, plasma arc cutting, waterjet cutting, and other similar techniques. Shearing is limited to size-reducing materials that are not too large or too strong for shears. Plasma arc cutting adds risk to operations due to the flame inherent in the operation and the high temperatures involved. As previously stated, waterjet cutting adds an undesirable secondary waste system to the process. The development of cryogenic cutting will produce a cutting technique that is widely applicable and produces no secondary waste stream.

This technology will be used to reduce the size of materials and eliminate the secondary waste stream inherent in waterjet cutting. This project is still in a research phase and the cost-benefits are not quantified. Cost savings will be dependent on the specific application. However, savings will be driven primarily by the reduction in secondary waste-stream handling and disposal costs.

\section{Stage Of Development}

Prototype 


\title{
Collaborative Partners
}

Cryela Cryogenics B.V.

\section{Affected Industries}

DOE, DOD, mining, agriculture, manufacturing, waste management, and emergency response.

\subsection{COOPERATIVE TELEROBOTIC RETRIEVAL}

\section{Technical Contacts}

Kevin M. Croft (208) 526-8276 ekm@inel.gov

Scott M. Allen (208) 526-8215 sca@inel.gov

Reva Hyde (208) 526-0741 rva@inel.gov

Environmental Restoration Technology Department

Technology Transfer Office Contact

Paul Grahovac (208) 526-3488 pg2@inel.gov

\begin{abstract}
A telerobotic system has been developed to deploy manipulators, a contamination control unit, digface characterization equipment, sizing equipment, and other equipment required to retrieve buried waste or objects.

The remotely operated vacuum system removes soil and debris from around the object of interest. End effectors are used with the vacuum system to break up hard soil, carefully clean around buried objects, and ensure that large sheets of plastic and other objects do not plug the system. The vacuumed debris is placed in a transport container for subsequent removal and treatment. A system has been developed to control the required number of motions.

Various buried waste support technologies are integrated for deployment from a common platform, the gantry crane. The gantry crane is equipped with two cooperative telerobotic manipulators \{multimanipulator capability (MMC) \}, a telescoping mast, and a hoist from which the ancillary tools will be deployed.

The crane system can be used to support archaeological excavations (soil vacuum and sundering equipment), digface characterization \{INEL radiation, magnetics, and volatile organic compound (VOC) sensors, and Pacific Northwest Laboratory radar\}, dust suppression (INEL Contamination Control Unit), waste retrieval from the excavation (slings and/or grapple), and delivery of waste to the proposal transport system (hydraulic grapple to interface with excavator ITM).
\end{abstract}

\section{Stage Of Development}

Prototype 


\title{
Intellectual Property Status
}

Industry participation is anticipated in the development of a delivery system to be used in this project. Advances made in the expansion of a control system will be transferred to the control system manufacturer partnered in these activities.

\section{Affected Industries}

DOE, DOD, mining, agriculture, manufacturing, waste management, and emergency response.

\section{Competitive Advantage}

The system has been developed to demonstrate that available technology can be deployed in a realistic waste remediation scenario by a cooperative telerobotic platform. The system has been developed with versatility to allow Environmental Management to challenge its capabilities for data to support the claims that such activities can be performed remotely to minimize worker exposure.

\section{The Research}

- Identified a delivery system to transport equipment, such as robotic manipulators, to be buried waste pit site

- Designed and fabricated a remotely operated vacuum system to remove soil and debris from around objects to be surgically removed

- Developed a control system capable of controlling the required axes of motion

- Developed the capability to control two robotic manipulators used in cooperative activities.

This technology provides improved safety to the worker involved in operations in hazardous environments. Cost savings are expected through the increased worker productivity experienced by removing the worker from the hazardous environment.

Many operations currently performed in hazardous environments are approximately $50 \%$ efficient due to the time and expense required for workers to don personal protective equipment and work for limited amounts of time in the environment. Additionally, cost savings will be realized by reducing the need for personal protective equipment required to work in a hazardous environment and the decontamination of disposal of the equipment. For work in hazardous environments, the buddy system is invoked, requiring redundant personnel for most operations. By allowing the work to be performed remotely, the buddy system and its associated costs will not be required.

\subsection{THE PINS CHEMICAL ASSAY SYSTEM}

\section{Technical Contact}

\author{
A. J. Caffrey \\ Idaho National Engineering Laboratory \\ P.O. Box 1625 \\ Idaho Falls, Idaho 83415-2114 USA \\ Phone: (208) 526-4024
}




\section{Description of Technology}

The Portable Isotopic Neutron Spectroscopy (PINS) Chemical Assay System is a field non-destructive evaluation tool to identify the contents of munitions and chemical storage containers safely and reliably. Typical assay times range from 100 to 1000 seconds.

PINS employs neutron radiation from a small radioisotopic source as a probe of an item's fill. The chemical elements inside the item are revealed by their characteristic gamma-ray signature, measured by a high-resolution HPGe spectrometer. The system computer then infers the fill compound or mixture from the elemental data.

\section{Testing \& Reliability}

PINS has been extensively tested with live CW agents and high explosives, including nerve agents GA, GB (sarin), and VX; blister agents HD, IIN, IIT (mustard gases) and L (lewisite); and explosives Composition B, RDX and TNT. The system can also identify items filled with military screening smokes, such as titanium tetrachloride (FM) and white phosphorus (WP), and it can identify practice munitions filled with water, concrete, or sand.

Since 1992, PINS has been used by the U. S. Army to reliably identify hundreds of suspect munitions recovered from burial sites and firing ranges.

\section{Equipment Features}

\section{Gamma-ray spectrometer}

Detector: n-type high-purity germanium (HPGe)

Efficiency: $>40 \%$ at $1332 \mathrm{keV}$

Resolution: $<1.95 \mathrm{keV}$ at $1332 \mathrm{keV}$

form"

$\mathrm{LN}_{2}$ usage: 1 liter per day

\section{Electronics}

Model: EG\&G Ortec Nomad Plus MCA

AC power: 15 watts, $110 / 220 \mathrm{VAC}, 50-60 \mathrm{~Hz}$

Battery lifetime: $>8$ hours

Dimensions and weights

Gamma-ray detector

Length $58 \mathrm{~cm}$ (23 in)

Width $15 \mathrm{~cm}(6 \mathrm{in})$

Height $22 \mathrm{~cm}$ (7 in)

to 70 in)

Weight $4.2 \mathrm{~kg}(9 \mathrm{lb})$
Electronics

$45 \mathrm{~cm}$ (18 in)

$35 \mathrm{~cm}$ (14 in)

$16 \mathrm{~cm}$ (6 in)

$12 \mathrm{~kg}(25 \mathrm{lb})$
Neutron source

Isotope: californium-252 Half-life: 2.6 years

Strength: 5 micrograms (2.7 millicurie or $99 \mathrm{MBq}$ )

Capsules: welded 304L SS, certified at IAEA "special

Shipping container: Type A, specification 7A

Notebook computer

Model: Compaq Elite 4/50E CPU: Intel 486

RAM: 8 megabytes

Hard disk: 250 Mbyte (removable)

$\frac{\text { Computer }}{28 \mathrm{~cm}(11 \mathrm{in})}$
$21 \mathrm{~cm}(8 \mathrm{in})$
$5 \mathrm{~cm}(2 \mathrm{in})$

$2.9 \mathrm{~kg}(6.4 \mathrm{lb})$

$32 \mathrm{~kg}(70 \mathrm{lb})$

Shipping weight: $248 \mathrm{~kg}(547 \mathrm{lb})$ total; six containers; heaviest container weighs $<35 \mathrm{~kg}$ (77 lb 


\section{LAWRENCE BERKELEY NATIONAL LABORATORY}

\subsection{GAMMA-2N GENERATION OF E+-E- ANNIHILATION RADIATION FROM NITROGEN}

\section{Technical Contact}

Norm Madden

Phone: (510) 486-5130

Fax: (510) 486-4122,

E-mail: NWMadden@lbl.gov

\section{Description of Technology}

The Measurement Science Group at LBNL considers the technique proposed by Luis Alvarez (Nobel prize in physics) to be the most promising nuclear avenue for land mine detection and classification. The N14 (Gamma, 2 n) N12, e+-e-reaction appears to be selective enough with respect to other elements. This reaction has an adequate interaction cross section, an attainable incident energy threshold, a reaction product (N12) with a unique half life, and an emitted radiation ( $511 \mathrm{KeV}$ photon) which has both a moderate range in soil and can be imaged with the aid of mechanical collimation. In particular the annihilation of the positron within a few half lives of the N12 will provide a unique signature demonstrating the presence of nitrogen. It is the consensus here that the best approach would be to perform a thorough simulation of the physics, and a companion set of experimental measurements with a prototypical detector array. The most likely detector candidate is a mechanically collimated germanium detector ensconced in a halide scintillator based Compton suppression shield. This combination will provide both the necessary energy resolution and adequate spatial resolution. Halide scintillators and germanium detectors are both readily commercially available. At present there is no funding for this activity and no system available for deployment.

\section{Technical Contact}

Henry Rutkowski

Phone: (510) 486-4113

Fax: (510) 486-5788

E-mail: HLRutkowski@LBL.Gov

\section{Description of Technology}

The incident photon necessary for this application requires at least a $40 \mathrm{MeV}$ electron beam. A pulsed beam will be necessary to exploit the unique half life of the N12. Commercially available medical accelerators exist which routinely reach these energies (Varian). However, a key problem for the field application is construction of a compact mechanically robust accelerator that will generate the required electron energy. Accelerating gradients as high as $7 \mathrm{MeV} / \mathrm{m}$ have been achieved in superconducting electron linacs. This implies a very long machine even if one uses a technology not easily deployable in the field. Perhaps a recirculating machine such as a betatron or microtron can be used. LBNL has a world class capability in novel accelerator structure development. No funded activity in this area exists and no accelerator is available for field use. 


\title{
4.2 MINE DETECTION AND IDENTIFICATION WITH A DUAL ELECTROMAGNETIC AND ACOUSTIC SYSTEM
}

\section{Technical Points of Contact:}

\author{
H. F. Morrison \\ LBL/UCB
}

(510) 642-3804

Fax (510) 642-3805

HFMorrison@LBL.Gov

Ki Ha Lee

LBNL

(510) 486-7468

Fax (510) 486-5686

KHLee@LBL.Gov

Louise Pellerin

LBNL

(510) 486-5026

Fax (510) 486-5686

LPellerin@LBL.Gov

Alex Becker

LBL/UCB

Jamie Rector

LBL/UCB(510) 643-7820

JWRector@LBL.Gov or rector@eg426.berkeley.edu

\section{Description of Technology}

We propose to develop a dual electromagnetic (em) and acoustic system to detect mines emplaced in the upper meter of soils and unconsolidated surficial materials. The em system will respond to electrical conductivity and dielectric properties of an object and the acoustic system will respond to its acoustic impedance (density velocity product) contrast with the surroundings. The systems operate independently but the data is acquired simultaneously and the results are merged in the detection-identification algorithms. The joint electrical-acoustic properties greatly enhance the reliability of the detection process. We plan to use neural network detection-identification algorithms with site-specific data such as soil conditions, mine types and burial methods used to train the neural networks. This approach will allow rapid discrimination of false targets and will allow us to incorporate the high degree of site variability.

\section{The E.M. System}

The em system utilizes a window of the electromagnetic spectrum from $1.0 \mathrm{MHz}$ to $100 \mathrm{MHz}$. This window has not been used for existing em or radar systems to detect small objects in the upper meter of the ground. Radar can be used successfully in this depth range if the ground is resistive but most soils are, in fact, conductive $(0.01$ to $1.0 \mathrm{~S} / \mathrm{m})$. For example, in a soil of $0.2 \mathrm{~S} / \mathrm{m}$ the maximum range for current ground penetrating radar (GPR) is only $17 \mathrm{~cm}$. Other factors controlling the resolution of radar for small 3-D objects is the spatial averaging inherent in the electric dipole antenna and the scattering caused by soil inhomogeneities 
of dimensions comparable to the wavelength (and antenna size). To obtain maximum resolution, it is desirable to use the highest frequencies (small antenna) but the scattering is large and target (mine) identification is poor. Lower frequencies may still resolve the mine with less scattering interference but resolution is lost because of antenna size.

At frequencies lower than those used for radar, the em fields satisfy a diffusion equation. Depth of investigation is controlled by skin depth rules and lateral resolution of an object depends on spatial mapping of the response, again the highest resolution being achieved when the detector dimensions are less than the response pattern of the target. For the typical soils referenced above, frequencies in the range of $1.0 \mathrm{MHz}$ to $100 \mathrm{MHz}$ are required for skin depths of $0.5 \mathrm{~m}$. Most previous work in em induction has utilized loop sources and receivers, which solves the problem of antenna size, but loses the resolution that could be achieved if a small electric antenna could be used. Finally, another common approach in the inductive regime is to measure the surface impedance (ratio of electric to magnetic fields) which is fully diagnostic of the subsurface conductivity and dielectric but which is no longer strongly dependent on the nature of the source of the em fields.

Our analysis of the many factors which control the radar and diffusive (induction) responses of inhomogeneous grounds has been undertaken with a numerical simulator. The simulator can determine the electromagnetic response, for the desired bandwidth, of any transmitter receiver configuration over any 3-D model of the ground. We have previously used the simulator for designing systems for the $20-30 \mathrm{~m}$ depth range, but we recently extended its range to over $100 \mathrm{MHz}$ and have applied it to the problem of detecting a small dielectric body buried in a half space of $100 \mathrm{ohm}-\mathrm{m}$. We calculated the scattered electric and magnetic fields for a variety of sources and investigated the feasibility of several sensors that do not seem to have been used before in this band.

These simulations indicate that the detection and characterization of a mine is feasible using new systems with the following features:

1. Swept frequency domain operation $1 \mathrm{MHz}$ to $100 \mathrm{MHz}$.

2. Multiple detector array with (relatively) distant source.

3. Loop source (possibly multiaxis for variable polarization of the illuminating field).

4. Electric and magnetic detectors (again multiaxis) for tensor impedance and field polarization measurements.

5. The use of torroids for electric field detection (a 3 " torroid of 100 turns produces adequate output for the field of a loop source $3 \mathrm{~m}$ away of unit moment and over the 1-100 $\mathrm{MHz}$ bandwidth).

6. The use of multiturn air core loops for magnetic field detectors.

7. Input impedance measurements of the loop antenna itself measures ground

8. impedance (and might provide the simplest system of all).

The proposed system is basically an array interferometer. It has the frequency range to detect objects in resistive ground thus overlapping radar but it can also operate at lower frequencies in conductive ground and respond to targets beyond the radar range. The resolution needed for small target detection is achieved with compact electric and magnetic field detectors. Broad bandwidth and the use of an array of sensors provides powerful data processing approaches using target sensitive filters and background noise removal schemes.

A field system developed from these principles would be light-weight, could be carried above the ground surface (non-contacting), and could be adaptively "tuned" to adjust for changing background (soil type). 


\section{The Acoustic System}

We propose to investigate a novel acoustic mine detection system to complement the em system. For the acoustic system we plan to exploit the little-known fact that the acoustic velocity in the upper meter of unconsolidated surficial materials (in which mines are usually placed) is substantially less than the velocity of sound in air. Our studies of near surface properties using vertical seismic profiling and very shallow reflection profiling have demonstrated that this surficial layer has velocities of less than $100 \mathrm{~m} / \mathrm{sec}$. This layer in fact causes major problems for conventional seismic surveying because it is highly attenuating and because its variability and low velocity introduces substantial travel time delays in arrivals from depth. Great effort is expended to reduce the impact of the surface layer including the burial of geophones beneath it. The problems for deep investigation are, however, unrecognized advantages for investigating the layer itself.

First, the wavelength of a $1 \mathrm{kHz}$ wave with a velocity of $100 \mathrm{~m} / \mathrm{sec}$ is only $10 \mathrm{~cm}$. This is an ideal wavelength for resolving objects of the same order of dimension. Second, although the attenuation at $10 \mathrm{kHz}$ is severe if signals must pass through the entire soil or weathered layer (many meters) it is not a serious problem for total path lengths of a meter or two. Finally, because the velocity contrast from air to soil is greater than one, incident acoustic energy from the air is refracted towards the vertical and surface waves (critical angle refracted waves) are not generated.

Under these circumstances a pulse source above the ground (i.e. a loudspeaker) with a $50-2000 \mathrm{~Hz}$ bandwidth will succeed in transmitting enough energy through the ground interface to achieve significant reflections from subsurface impedance contrasts. These reflections in turn can be detected with receivers coupled to the ground surface or possibly suspended above the surface. One of the principal difficulties with acoustic detection subsurface anomalies using sensors above the ground surface is the large reflection (due, primarily to the large density contrast) at the air/ground interface. However, recent advances in acquisition technology make this problem more tractable. State-of-the-art seismic acquisition systems have 24 bits of precision, which is more than enough dynamic range to detect subsurface reflections. The use of large detector arrays along with conventional seismic array processing techniques (multichannel filtering, migration) can be used to enhance the weaker subsurface reflections and distinguish them from air-ground modes. Additionally, novel techniques such as laser interferometry may provide a means to record ground motion with high fidelity without actually coupling detectors to the ground surface. We propose to evaluate different techniques for sensor deployment including ground-coupled accelerometers, air-coupled directional microphones, and laserinterferometry.

The em and acoustic systems would share a common platform: The detectors grouped along a horizontal line or rectangular plane which is moved over the ground by a boom and the sources (loud speaker and em loop) positioned above the detectors by another boom. Since the frequency bands for the two systems are so different, we anticipate no technological problems in operating both simultaneously.

\subsection{COLORIMETRIC LIGAND-BASED BIOSENSORS}

\section{Technical Contact}

Deborah Charych

Materials Sciences Division

510-486-6430

Fax 510-486-4995

DHCharych@lbl.gov 


\section{Description of Technology}

Sensitive and selective molecular recognition is important throughout biology. Certain organisms and toxins use specific binding at the cell surface as a first step towards invasion. A new series of biomolecular materials, with novel optical and interfacial properties have been designed to sense molecular recognition events. These polymers, based on diacetylenic lipid monomers, are shown to undergo blue to red chromatic transitions in response to virus and toxin binding to the surface of the material. Synthetic or natural lipid molecules such as gangliosides can be incorporated into the polydiacetylene matrix. The materials selfassemble to form thin Langmuir-Blodgett films or bilayer vesicles. Polydiacetylenes incorporating the Clinked glycoside of sialic acid change color from blue to red in the presence of influenza virus through interactions with the viral hemagglutinin. Gangliosides that bind to toxin molecules can also be incorporated into the diacetylenic matrix. Polydiacetylenic lipid membranes offer the possibility of a general 'litmus test' for molecular recognition at the surface of a membrane. The advantage of these supramolecular assemblies is that signal transduction - the color change - is directly linked to molecular recognition. The approach circumvents the need for secondary visualization reagents such as enzyme-linked antibodies.

\section{Technical Status}

The conceptual idea of directly detecting biological binding by a color change of the ligand-bearing polymer has been demonstrated and published in key scientific journal (eg. Science, Journal of the American Chemical Society, Chemistry \& Biology). Several companies have expressed interest in the idea and commercial development is likely although not in the immediate future. extension of the concept to a wide variety of ligand/receptor, antibody/antigen, enzyme/substrate, etc interactions has only been partially demonstrated. Funding to date has been limited to year by year grants and currently there is no long term funding (although several grants are pending). All of the equipment required to perform the application is available in the Charych lab or within the division. However, certain characterization tools such as an atomic force microscope dedicated to polymer membranes and a light scattering instrument dedicated to polymer membrane liposomes are unavailable.

\subsection{DATA FUSION TECHNIQUES}

\section{Technical Contact}

Arie Shoshani

(510) 486-5171

AShoshani@LBL.Gov

\section{Description of Technology}

Imaging technology used and developed in the Information and Computing Sciences Division (ICSD) includes: image enhancement techniques, object identification based on known characteristics of the mines, and terrain specific characteristics. Previous projects have shown that object identification based on known characteristics is effective and accurate. We propose to apply such imaging methods to integrated data from the geophysical sensors sensors described in a separate article for detection of mines.

Data Management techniques used and developed in the Information and Computing Sciences Division (ICSD) includes the support of structures and operators over two-dimensional data, disk based parallel algorithms to achieve efficient processing, and probabilistic correlation of data. The amount of data to be analyzed and correlated will be sufficiently large that effective methods of processing the data will be required 
in order to achieve near real time evaluation of the data.

The overall goal is to achieve accurate detection of land mines from airborne geophysical data. The hope is that the combination of technologies mentioned above will produce results with very few false negatives (missed land mines) in the order of $<1-2 \%$, at the cost of having reasonable number of false positives (objects that are not land mines) in the order of $<10 \%$.

\subsection{NEURAL NETWORKS}

\section{Technical Contact}

Edward Theil

(510) 486-7501

Fax (510) 486-5936

EHTheil@LBL.Gov

\section{Description of Technology}

One of the most promising approaches to "intelligent" processing of data from atomic particle interactions is the use of a so-called hybrid neural-fuzzy logic system to model the rules used by an expert observer in identifying particular events. This approach combines the power of neural network pattern recognition with the ability of fuzzy controllers to resolve semantic ambiguities. As such, it is a promising new tool for the analysis of complex signals.

Regardless of the particular method of detection used, all such methods have two principal components. The first is the hardware detector, designed initially according to principles of physics. The second is the intelligent analysis of the signals acquired by the detector. This component, designed according to engineering principles, relies primarily on software.

Lawrence Berkeley National Laboratory has a long history of expertise in both these areas. In terms of computer analysis of signals from atomic and nuclear interactions, the Engineering division has extensive experience in analyzing such signals in nuclear science, high-energy physics and nuclear fusion. In particular, along with our competence in traditional signal processing, we have used neural networks to identify events and patterns in areas as diverse as the Human Genome Project and positron emission tomography.

\subsection{NEUTRON SOURCES}

\section{Technical Contact}

Ka-Ngo Leung

(510) 486-7918

Fax (510) 486-5105

KNLeung@LBL.Gov

\section{Description of Technology}

One approach is the use of RF cusp field ion sources to produce high-energy beams of D+. Currents up to $20 \mathrm{~mA}$ DC with monatomic species have been achieved in $2 \mathrm{~mm}$ apertures for a geophysical logging program. 
The high current allows generation of D-D neutrons, which are born at lower energy than D-T neutrons, because the extra current can be used to make up for the lower cross section. An LDRD program continues this work.

\section{Technical Contact}

Ian Brown

(510) 486-4174

Fax (510) 486- 4374

IGBrown@LBL.Gov

\section{Description of Technology}

Another proposed method for generating neutrons is acceleration in a pulsed high voltage sheath. This builds on LBNL's capabilities in plasma surface modification and ion implantation. Pulses of $1-10 \mu$ s at $100 \mathrm{kV}$ in deuterium plasmas should allow creation of D-T neutrons in the wall material. A compact plasma source of neutrons would result that uses no complex accelerator structures. No funding is available for this yet.

\subsection{CHEMICAL/BIOLOGICAL TECHNIQUES}

\section{Technical Contact}

J.C. Hunter-Cevera, Head

Environmental Biotechnology Program

Phone: (510) 486-7359

Fax: (510) 486-7152

JCHunter-Cevera@LBL.Gov

\section{Description of Technology}

Bacteria can degrade TNT under several different conditions. It is feasible to attach a lux gene that would light up when the TNT was being degraded and monitor off line with sensors similar to Gary Saylors work on lysimeter experiments using genetically engineered microorganisms to degrade hydrocarbons in soil. One could screen for "natural organisms" such as spore forming bacteria or fungi that are generally regarded as safe and can degrade the explosives. If they are encased with plastic you will need to possibly genetically engineer a bifunctional microbe that could use both the plastic material and TNT as a substrate for metabolism. To detect the mines, a receptor-ligand approach could be employed that would involve a color reaction with the mines that would change the color of the soil, indicating where the mines are buried. The chemical could be sprayed from the air and then viewed via infrared or some other wavelength. There is no funded research at LBNL in this area but research capabilities are available to address these approaches.

\subsection{SQUIDS}

\section{Technical Contact}

Prof. Alex Pines 
Materials Sciences Division

Lawrence Berkeley National Laboratory

Phone: (510) 6421220

Fax: (510) 6425744

A_Pines@lbl.gov

\section{Description of Technology}

Our high-Tc superconductor-based SQUIDs, which are developed in the research program of John Clarke, are the most sensitive in the world. Because they are liquid nitrogen (rather than liquid helium) cooled, they can be brought close to room temperature objects for increased sensitivity. For example, they can be used as very sensitive detectors of metals by using measuring induced eddy currents. However, they do not seem practical by themselves for mine detection due to the high concentration of metallic "clutter" in actual minefields. In addition, SQUID technology cannot detect mines that are primarily non-metallic in composition.

SQUID-NMR Detection Of Nitrogen In Explosives- For completeness we mention this new technology for detecting nitrogen using nuclear quadrapole resonance (NQR) spectroscopy with SQUID detection. This method does not require an applied magnetic field. Although it does not seem practical now for field sensing of $\mathrm{N}$ in buried explosives, it might be practical for a fixed system for examining baggage or passengers for explosives or for mine detection after considerable R\&D. It should be noted that the technology is safe to use on human subjects, which would differentiate it from gamma ray, neutron beam, and X-ray-based approaches. A brief technical description follows.

Nuclear magnetic resonance with Superconducting Quantum Interference Device (SQUID) detection is proposed for field detection of explosives. This proposal is based on SQUID-NMR technology developed at LBNL which has recently been applied to the detection of N-14 in solids in an ongoing collaboration with the Naval Research Laboratory (NRL). The research program would involve obtaining the SQUID-NMR spectra in zero field for nitrogen in a variety of explosive materials in order to investigate the uniqueness of the spectra and the corresponding fingerprinting capability, the influence of proximate objects (including metals, which might limit (sensitivity) on the SQUID delectability, the problems of size scale-up and the technical requirements of in-field (e.g. airport etc.) cryogenics. Our current SQUID detectors require liquid helium, but application of high sensitivity, low-noise, high-Tc-superconductor based SQUIDs, which have been developed at LBNL and only require liquid nitrogen for

cooling, will be straightforward. At the same time we could also investigate non-SQUID classical NQR techniques which are less sensitive but are already under vigorous investigation at NRL.

\section{Technical Status}

The application of SQUID-NMR to the detection of nitrogen-14 in solids is in the early development stage. In recent collaborative work with Allen Garroway of the Naval Research Lab, the detection of nitrogen in cocaine and in a dipeptide was reported for the first time. A patent disclosure has been submitted through the LBNL patent office. In a related technology, LBNL is developing a scanning SQUID-NMR microscope, which might in itself be interesting for small packets containing molecules with nitrogen. Currently, the collaborative LBNL/NRL development work is funded by basic research funds at the respective labs on an ongoing basis. Unique SQUID-NMR equipment at LBNL and non-SQUID classical NQR equipment at NRL are available for this project. 


\subsection{A D-D NEUTRON SOURCE FOR MINE DETECTION USING THERMAL NEUTRON ANALYSIS}

\section{Technical Contact}

Christine M. Celata

50-149

E.O. Lawrence Berkeley National Laboratory

1 Cyclotron Road

Berkeley, CA 94720

Phone: (510) 486-7740

Fax: (510) 486-6003

E-mail: CMCelata@LBL.gov

\section{Description of Technology}

A pulsed D-D neutron source is being developed at LBNL which can greatly enhance the abilities of mine detection systems based on thermal neutron analysis (TNA). Most mines buried worldwide are small (antipersonnel) and contain a minimum amount of metal content in order to avoid detection. Systems based on the detection of metals, while they can be successful in quickly locating mines, frequently trigger on shrapnel, bullets and other metal debris as a result of the increased sensitivity required to detect the small metal content, giving an unacceptably high false alarm rate. More specific systems based on the thermal neutron analysis (TNA) technology detect the nitrogen in mine explosives, and have demonstrated their capability to detect mines and distinguish them from clutter material. However, the detection of the smallest mines requires unacceptably long inspection times not compatible with clearing operations. Detection time is ultimately limited by the maximum acceptable count rate in the gamma-ray detectors before the necessary spectroscopic features are debased by pileup and other electronic effects. Present systems using isotopic neutron sources operate at this limit. The limit can be exceeded using a pulsed source. During the pulse, gamma ray detectors can be gated off, so that the very high count rate due to neutron interactions in the crystal, and gamma rays stimulated by the neutrons through inelastic scattering process, can be tolerated. After the end of the neutron pulse, the neutrons are quickly thermalized, and by waiting a few tens of microseconds, a count rate due only to thermal neutron capture gamma rays is reached. This is fully compatible with the capabilities of the detectors and electronics, and yields the best possible signal to background (and/or noise) ratio. While offthe-shelf commercial D-T neutron generators are available which produce $\sim 5 \times 10^{8}$ neutrons per second, the lifetime of the target is unacceptably low ( $<300$ hours). The same neutron generator with a deuterium target will last much longer, but the yield will be only $\sim 5 \times 10^{6}$ neutrons/second-- not enough to compete favorably with the isotopic source. A LBNL high flux ion source under development promises a factor of 100 or more increase in flux for the D-D source (the same is true for the D-T source), making it the first pulsed source capable of producing the necessary flux, and the source of choice for this application. The LBNL source is a high current density rf multicusp ion source developed for high energy and nuclear physics. An rf antenna is used to ionize plasma in a volume source. Using a magnetic filter, a beam of deuterons which is $80-90 \%$ monatomic can be produced. The ions are then accelerated to $\sim 100 \mathrm{keV}$ onto a deuterated target. A 2-inch diameter source has been shown to produce an ion flux predicted to yield $10^{8} \mathrm{D}-\mathrm{D}$ neutrons per second. A larger flux could be produced simply by scaling the diameter of the source. The lifetime of the source is believed to be much longer than the Penning ion sources used in conventional neutron tubes, since the rf antenna is very rugged. It should be mentioned that the pulsed D-D source offers significant environment and safety advantages over the isotopic sources, which also translates into convenience of use and transport. The source can of course be switched off, and there is no danger of exposing or dispersing a radioactive source, should an explosion occur. The availability of the proposed high yield neutron generator would extend the applicability of the TNA based system to the detection of small anti-personnel mines and would reduce the false alarm rate. We propose to develop this source as a component of a mine detection system. One 
possible system is built by SAIC, and uses metal detectors, with TNA as the confirmatory sensor. R\&D of the D-D neutron tube would be done at LBNL, probably in collaboration with LANL.

\title{
Status
}

A 2 inch diameter version of the ion source has been built and tested for a neutron logging application. As mentioned above, this prototype easily produced the neutron flux needed for a mine detection system. The $100 \mathrm{keV}$ accelerator for the logging system is presently being fabricated. When this has been tested, it will be necessary to put the source and accelerator into a neutron tube and test the tube. For a mine detection project, this would probably be done in collaboration with LANL, where tubes and tube expertise resides. A collaboration with SAIC's Advanced Nucleonics Division in Santa Clara, CA is possible for this work, though LBNL will work to implement this technology in any other neutron system put forward by the sponsor. SAIC has produced a mine detection unit which integrates a TNA sensor and a primary metal detection sensor in a tele-operated vehicle.

\section{Funding}

The development of the ion source is presently funded by internal LBNL R\&D funds (LDRD) for a neutron logging application. The SAIC system tested at Fort A. P. Hill was funded by the U.S. Army Communications and Electronics Command and the present testing for UXO is being funded by the Office of Special Technology.

\subsection{MINE DETECTION WITH AN RF ELECTROMAGNETIC SYSTEM}

\section{Technical Contacts}

\author{
H. Frank Morrison, Ki Ha Lee, and Alex Becker
}

\section{Description of Technology}

We propose to develop radio frequency electromagnetic (em) system to detect mines emplaced in the upper meter of soils and unconsolidated surficial materials. The em system will respond to electrical conductivity and dielectric properties of an object. The em system utilizes a window of the electromagnetic spectrum from $500 \mathrm{kHz}$ to $30 \mathrm{MHz}$. This window has not been used for existing em or radar systems to detect small objects in the upper meter of the ground. Radar can be used successfully in this depth range if the ground is resistive but most soils are, in fact, conductive $(0.01$ to $1.0 \mathrm{~S} / \mathrm{m})$. For example, in a soil of $0.2 \mathrm{~S} / \mathrm{m}$ the maximum range for current ground penetrating radar (GPR) is only $17 \mathrm{~cm}$. Other factors controlling the resolution of radar for small 3-D objects is the spatial averaging inherent in the electric dipole antenna and the scattering caused by soil inhomogeneities of dimensions comparable to the wavelength (and antenna size). To obtain maximum resolution, it is desirable to use the highest frequencies (small antenna) but the scattering is large and target (mine) identification is poor. Lower frequencies may still resolve the mine with less scattering interference but resolution is lost because of antenna size.

At frequencies lower than those used for radar, the em fields satisfy a diffusion equation. Depth of investigation is controlled by skin depth rules and lateral resolution of an object depends on spatial mapping of the response, again the highest resolution being achieved when the detector dimensions are less than the response pattern of the target. For the typical soils referenced above, frequencies in the range of $500 \mathrm{kHz}$ to $30 \mathrm{MHz}$ are required for skin depths of $0.5 \mathrm{~m}$. Most previous work in em induction has utilized loop sources and receivers, which solves the problem of antenna size, but loses the resolution that could be achieved if a 
small electric antenna could be used. Finally, another common approach in the inductive regime is to measure the surface impedance (ratio of electric to magnetic fields) which is fully diagnostic of the subsurface conductivity and dielectric but which is no longer strongly dependent on the nature of the source of the em fields.

Our analysis of the many factors which control the radar and diffusive (induction) responses of inhomogeneous grounds has been undertaken with a numerical simulator. The simulator can determine the electromagnetic response, for the desired bandwidth, of any transmitter receiver configuration over any 3-D model of the ground. We have previously used the simulator for designing systems for the $20-30 \mathrm{~m}$ depth range, but we recently extended its range to over $100 \mathrm{MHz}$ and have applied it to the problem of detecting a small dielectric body buried in a half space of $100 \mathrm{ohm}-\mathrm{m}$. We calculated the scattered electric and magnetic fields for a variety of sources and investigated the feasibility of several sensors that do not seem to have been used before in this band.

These simulations indicate that the detection and characterization of a mine is feasible using new systems with the following features:

1. Swept frequency domain operation $500 \mathrm{kHz}$ to $30 \mathrm{MHz}$.

2. Multiple detector array with (relatively) distant source.

3. Loop source (possibly multiaxis for variable polarization of the illuminating field).

4. Electric and magnetic detectors (again multiaxis) for tensor impedance and field polarization measurements.

5. The use of torroids for electric field detection (a 3" torroid of 100 turns produces adequate output for the field of a loop source $3 \mathrm{~m}$ away of unit moment and over the $0.5-30 \mathrm{MHz}$ bandwidth).

6. The use of multiturn air core loops for magnetic field detectors.

7. Input impedance measurements of the loop antenna itself measures ground impedance (and might provide the simplest system of all).

The proposed system is basically an array interferometer. It has the frequency range to detect objects in resistive ground thus overlapping radar but it can also operate at lower frequencies in conductive ground and respond to targets beyond the radar range. The resolution needed for small target detection is achieved with compact electric and magnetic field detectors. Broad bandwidth and the use of an array of sensors provides powerful data processing approaches using target sensitive filters and background noise removal schemes.

A field system developed from these principles would be light-weight, could be carried above the ground surface (non-contacting), and could be adaptively "tuned" to adjust for changing background (soil type). 


\section{LAWRENCE LIVERMORE NATIONAL LABORATORY}

\subsection{THERMAL DETECTION/INFRARED}

A buried mine acts as a impermeable barrier to the normal movement of soil moisture, preventing gravimetric flow downward during precipitation; and upward movement by capillary action during times of surface moisture depletion. As a consequence, the normal water distribution of soil is altered by the presence of the mine. In general, the soil above a mine is usually drier and more porous than homogeneous soil (at the same depth) surrounding the disturbed area where the mine was emplaced. This does not always hold true right after rainfall or when the soil is saturated.

Soil water content has marked effects on thermal properties to include heat capacity and conductivity, and electromagnetic reflection, transmission and emission characteristics. Infrared emissivity and reflectance are strongly affected by moisture content and hence are potentially useful phenomena for recognizing moisture and thermal anomaly patterns indicative of mined areas.

The disturbed soil over a mine cools faster (and more) as the sun retreats, and then heats faster than the surrounding undisturbed soil when the sun returns. There may be a difference of a few tenths of a degree, or several degrees depending on the moisture, solar load and soil composition. It is this difference in temperature which makes it possible to find buried mines in roads and non-foliated areas.

LLNL has been very active in electro-optical and (to a larger extent) infrared techniques for mine detection. Early work (1989-91) by Nancy Delgrande demonstrated the detection of mines by detecting the change in ground temperatures as a result of the placement of mines under the ground. The novel aspect of this work was the simultaneous use of mid-wavelength IR (MWIR, 3-5 $\mu \mathrm{m}$ ) and long wavelength IR (LWIR, 8-12 $\mu \mathrm{m}$ ). In a larger follow-on program supported by ARPA and U.S. Marine Corps, LLNL determined that this thermal technique would be limited to areas of low clutter. Since that time, the Army has used this type of system for the detection of mines in roads in Somalia. Other multispectral and multisensor experiments were conducted as part of this effort. A large test facility was established at the Nevada Test Site to support this experimental program. This work also emphasized the value of spectral analysis for mine detection.

\subsection{MULTI-SPECTRAL SCANNING/IMAGING}

\section{Technical Contact}

Mike Carter

925-423-1446

\section{Description of Technology}

Multispectral Scanners (MSS) obtain light energy measurements in the visible-near infrared and short wave infrared range, typically from 0.4 to 2.6 microns (also expressed as 400 to 2600 nanometers). Field scanners are able to measure the ultraviolet energy (.01 to .4 microns) but the MSS systems used from aircraft and satellite do not image the ultraviolet energy as too much of this energy is absorbed by the atmosphere. Most MSS systems collect data in broad band of about 100 nanometer width. New instruments called hyperspectral scanners (HSS) can collect data at 3-5 nanometer band widths which means that more distinct information can be collected about surface features that provides for the 
possibility of distinguishing pigments within a plant leaf or soil properties such as organic matter content or type of iron oxides.

LLNL is currently at work with ARPA on the detection of mines using infrared hyperspectral imagers. LLNL, using the LIFTIRS instruments, has provided important data for the development of a Hyperspectral Mine Detection system.

Thermal scanners operate on the same principle as MSS or HSS systems but the resultant images must be interpreted with due regard to the basic thermal radiation principles involved. LLNL has applied hyperspectral imaging to detect the disturbed soil above a buried mine.

LLNL has had at least two projects looking at the visible and near IR spectral regions. Measurements were made as part of the ARPA program, but no systems were developed. The CAMI program has developed a multispectral vis-near-IR camera system.

\subsection{RADAR}

Radar methods, particularly ground penetrating radar (GPR) were seen as very promising methods of detection, particularly if used in combination with other sensors. The main improvements that need to be made are feature extraction and target recognition. In addition, improvements to the hardware such as antenna design and bigger bandwidth are necessary. It is also necessary to develop the equipment into something that is lightweight (man-portable) and relatively inexpensive.

\section{Developments Underway Include}

- Algorithms and databases to enhance the reliable detection of mines by the identification of reliable features to yield a very high probability of detection with substantially-reduced false alarm rates.

- Fusion algorithms for arrays (e.g. as in synthetic aperture array - SAR - techniques) to enhance detection, identification and localization.

- Automated Target Recognition (ATR) algorithms to increase the detection rates, hence reducing costs, at a specified high probability of detection and low false alarm rate (these minimize the total costs by reducing unnecessary excavation).

\subsection{GROUND PENETRATING RADAR (GPR)}

A ground penetrating radar transmitter focuses microwave impulses into the ground. Buried objects such as metallic land mines reflect the microwave impulses back toward the GPR receiver. A moving GPR system can map the location of buried objects. For detecting buried mines (metallic) the GPR system is normally swept along in contact with the ground and operates within a frequency range of approximately $200 \mathrm{MHz}$ to 1 GHz. Plastic/non-metallic mines/object detection and identification is problematical. There are a number of GPR systems in prototype development, however, they consistently suffer from a high false positive detection rating. That is, they identify buried objects to be mines when in actuality they are various forms of metallic debris. Note that for every mine there may be as many as 2,000 metal fragments cluttering the area. 


\section{Ground Penetrating Radar - Standoff}

As part of the ARPA mine detection effort described above, LLNL worked with SRI to investigate the detection of mines using ground penetrating radar (GPR). LLNL demonstrated the detection of metal mines in a cluttered environment (where the IR systems did not work) using an ultra-wideband impulse radar system.

Lawrence Livermore National Laboratory (LLNL) has developed a side-looking, ground-penetrating impulse radar system that can eventually be mounted on a robotic vehicle or an airborne platform to locate buried land mines. The radar system uses LLNL-developed monocycle pulse generators which have been tailored to be most efficient for this application. Extensive field tests have been conducted on a mine field consisting of real and surrogate mines buried in natural vegetation. Results indicate that the LLNL GPR system consistently detects buried metal mines, but does not reliably detect plastic mines.

\section{Ground Penetrating Radar - Near field}

While GPR systems operated in close contact with the ground have been operated for some time, LLNL has worked in two different areas to change the nature of these systems. Work in the Engineering Research Division has been conducted to develop a synthetic aperture radar system for the 3-D imaging of buried structures, originally developed for inspection of civil structures. This work has direct application to mine detection using a vehicle mounted system.

\subsection{MICROPOWER IMPULSE RADAR (MIR)}

The Micro Impulse Radar (MIR) is an LLNL technology development which is applicable in at least two types of mine detection systems. The MIR transceivers can be used as the detection elements in the 3-D system described above, probably reducing cost, size and mass. The MIR technology can also be applied to a hand held mine detection system analogous to the hand held metal detector.

LLNL has developed a new extremely small radar sensor, the micropower impulse radar, for short range motion sensing and imaging applications. The micropower impulse radar was developed at LLNL in 1993. The compact MIR circuit module is a complete impulse radar transceiver on a chip and is constructed using low cost, off-the-shelf components. A variety of antennas have been designed for various applications. The transmit and receive antennas used for mine detection are resistively loaded cavity backed dipoles measuring about $4 \mathrm{~cm}$ on a side. With the antennas connected to the radar module, the entire system fits in a $4 \times 6 \times 10$ $\mathrm{cm}$ volume. The unit emits very low RF power in a broad RF band, resulting in low power consumption as well as making it difficult to detect in operation. The present unit has a $100 \mathrm{ps}$ rise time pulse yielding a range resolution of about $2 \mathrm{~cm}$ in typical soils. The entire radar package-drive electronics, transmit/receive antennas, range sweeping circuitry, and $9 \mathrm{~V}$ battery power for many hours of operation, fits in one hand, making it the smallest, lightest, and lowest cost radar known. Based on a pulser for emitting and detecting very low amplitude voltage impulses, the MIR has a sensitive detection window for accurate ranging that can be varied over time to provide the radar return information at different depths. Its ultra-wide band frequency range $(1-4 \mathrm{GHz})$ is near optimal for short range ground penetration $(<2$ feet $)$, and mine detection with reasonably high resolution $(\sim 1 \mathrm{~cm}$ in soil). It has been coupled with a laptop computer for completely portable image capture, processing, display, and analysis. This low cost opens up the possibility of using arrays of radar modules in a synthetic aperture mode or a diffraction-tomography based imaging mode. An array of radar units this small is consistent with implementation in a land robotic vehicle or a UAV. Input power requirement per unit is 6-12 volts at $8 \mathrm{~mA}$. Typical range in air is approximately 10 meters and range in soil is .2-.5 meters depending on soil conductivity and standoff range. 


\subsection{ACOUSTIC MINE DETECTION}

Advanced signal processing software is now available, which can be used with acoustic/seismic methods to produce tomographic images of mines and other objects several feet in front of the operator. This could distinguish a mine from other clutter and therefore reduce the false alarm rate. The hardware consists of an acoustic transducer array and a microprocessor. The returned acoustic signal is then interpreted in real time via a new calculational capability to reconstruct the three dimensional object causing the reflected acoustic signal. 


\section{LOS ALAMOS NATIONAL LABORATORY}

\subsection{ROBOTS PLATFORMS}

\section{Technical Contact}

John Moses

Phone: (505)667-7337

Fax: (505)665-4507

E-mail: jmoses@lanl.gov

\section{Technology Description}

We believe that it will be possible over the next few years to build robots which can operate autonomously and for extended periods of time in the natural environment. Such robots can then be used to do useful work, including work which is unpleasant or dangerous to humans. They will carry sensors specific to the application for which they are intended, and can be instrumented to record or report the location of objects found by the sensors. Equipped with suitable sensors, they could locate land mines. If desired, they could be designed to detonate the mines.

To build such robots will require a multidisciplinary effort drawing on (at least) physics, mechanical and electrical engineering, biology, and neuroscience. Because many of the design features of these devices functionally mimic biological systems, we use the term "biomorphic" to describe them. Devices built to date, the robots of Mark Tilden, use simple analog circuits to emulate the central pattern generators responsible for repetitive motion in invertebrates; the CPGs generate the gaits in simple walking robots. We believe that we can build on these designs, incorporating recent insights into the mechanics of legged locomotion and other technological advances to produce robots which are much more capable than any built to date. We will, at the same time, develop techniques to couple these robots with sensors and geolocation and navigation instrumentation which will allow them to survey an area for some target and to pinpoint the location of anything they find. We intend that these robots be inexpensive enough that large numbers of them could be used if needed. They could be sacrificial -- for example, a device which tumbles over a minefield and detonates pressure or tripwire activated mines could be used to go over an area before it is searched thoroughly by more sophisticated, sensor carrying robots, or by humans.

This technology is presently in a very early stage, and is not yet ready for as sensitive an application as mine clearing. We believe however, that it is ready for major advancement, and that with proper support useful devices might be built on the time scale of three to five years.

\section{Status}

This work has received support from internal program development funds and from the Deployable Adaptive Processing Systems (DAPS) project sponsored by DOE NN-20. We are presently collaborating with the Yuma Proving Ground to test and demonstrate the applicability of the technology for locating unexploded ordnance on test ranges, and we are actively exploring funding with several potential sponsors. We are optimistic that reasonable funding will be obtained. We expect internal funding to develop an advanced miniaturized fluxgate magnetometer and the geolocation and navigation systems for uses with these or other platforms. 
Laboratory scale preliminary devices are available for demonstration, and field prototypes are under development.

CC-196-484ig 128

\section{Biomorphic Machines A revolutionary approach to robotics}

-Versatlle, Inexpensive, survlval-orlented Ilving machines

- Intelligence achleved through artiliclal Nervous Netrm systems

- Applications

- Enwlronmental sensing, remedlation

- Securlty and malntenance

- Mine chetectlon and detonation

- Domestlc appllances

This solar-powered engineering prototype can be constructed at low cost (less than $\$ 20$ ).

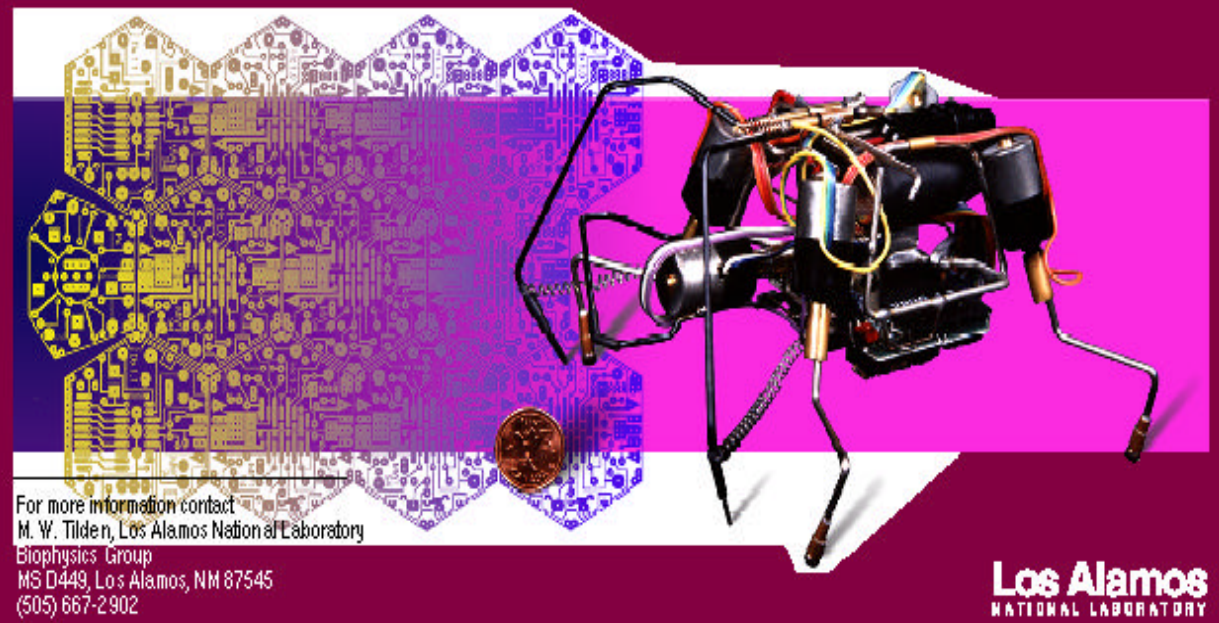

Fig. 8. Los Alamos Biomorphic Machine.

\subsection{NOVEL METHODS FOR NEUTRON-INDUCED-GAMMA DETECTION OF LAND MINES AND BURIED EXPLOSIVES}

\section{Technical Contact}

Mark M. Pickrell

Los Alamos National Lab

NIS-5, MS E540

Phone: (505) 665-5098

Fax: (505) 665-4433

E-mail: pickrell@safeguard.lanl.gov 


\section{Description of Technology}

Neutron-induced-gamma ray detection of unexploded ordnance (UXO) such as prompt gamma neutron activation analysis (PGNAA) and a subset of PGNAA, thermal neutron analysis (TNA) are appealing because they are extremely accurate. The neutron-based methods are specific to the explosives (specifically the nitrogen and other elemental content) rather than external attributes, so that they are sensitive to all forms of UXO (e.g. plastic mines). The technique is highly specific to explosives and cannot be easily mislead by either false positives (other buried objects that appear to be explosives) or by false negatives (failing to detect the explosive).

Because of this appeal, PGNAA and TNA methods have been attempted by several organizations for UXO detection, including SAIC, Sandia, and Idaho National Engineering Laboratory. The INEL system, in our judgment, is by far the most advanced and successful. However, PGNAA/TNA systems have not been deployed for land mine detection because they commonly suffer two significant limitations: the detector and neutron source must be very close to the sample (ground), and the measurement / detection takes a long time. A typical time to detect buried UXO is 15 minutes. Moreover, the detector and neutron source must actually touch the ground or the unknown UXO sample. The slow speed and required proximity have limited this method in practical application. Other organizations that have pursued this technology have not successfully addressed the time and proximity limitations.

At LANL we have addressed the time and proximity limitations directly. We assert that this method could be very practical and effective if the assay time were short and the distance longer. There are three physics reasons for the time and distance limitation: insufficient neutron source strength, insufficient gamma-ray detection speed in the gamma detectors, and insufficiently mapped, neutron-induced, gamma-ray lines from nitrogen, oxygen, carbon, and hydrogen. These three effects can be reduced to the single limitation of insufficient gamma-ray data rate. In order to detect buried explosives with certainty, a sufficient number of specific gamma rays must be detected (these gamma rays are the unique signature of the elements composing the explosive, primarily nitrogen). The number of required gamma ray counts must be sufficient to achieve precise counting statistics and to overcome the Compton continuum background. At present, sufficient gamma rays are counted only after 15 minute assays in close proximity. To improve this rate requires three changes: an increased neutron interrogation flux to induce more gamma-rays, the identification and measurement of more gamma-rays that are specific to the elements of interest, and an increase in the gamma detection throughput of the detectors. At LANL, we are addressing each of these three physics problems directly.

The principal objective is the development of an intense neutron source. Present PGNAA/TNA systems typically employ a $10^{6}$ neutron / second neutron source, usually an ${ }^{252}$ californium isotopic source. The ${ }^{252} \mathrm{Cf}$ source is reliable and steady, but it cannot be turned off. It is limited to a $10^{6} \mathrm{n} / \mathrm{s}$ intensity to limit the radiation dose; a higher intensity isotopic source would exceed safety and dose limits. By contrast, existing neutron generators can be turned off, but are unreliable and have a short lifetimes. In addition, moderately-sized systems only produce $10^{8} \mathrm{n} / \mathrm{s}$, which is insufficient. Higher intensity $\left(10^{11} \mathrm{n} / \mathrm{s}\right)$ neutron generators are prohibitively large, heavy, and have excessive power requirements. The requirement for this application is a neutron generator that can be turned off, has an output of $10^{11}$ neutrons / second, is reliable and long-lived, and has a moderate size, weight, and power requirement. An output of $10^{11} \mathrm{n} / \mathrm{s}$ is an increase of 5 orders of magnitude over existing systems and is sufficient to reduce assay times to nominally 1 second and remove the detector from the sample.

We have been investigating a source that potentially has these properties. It is the Inertial Electrostatically Confined (IEC) plasma source. In laboratory experiments it has already achieved $10^{10} \mathrm{n} / \mathrm{s}$ output. Experiments 
have been run reliably for thousands of hours. It can be turned off. The size of the system is 1 rack of electronics and a $60 \mathrm{~cm}$.-diameter, sealed vacuum sphere. Projected power requirements for a $10^{11} \mathrm{n} / \mathrm{s}$ source are between 1 and $10 \mathrm{~kW}$.

The IEC source was originally developed in the 1950s by Hirsch, and has subsequently been investigated by LANL, the University of Illinois, and INEL. It is a spherically symmetric electrostatic accelerator that exploits the deuterium-tritium fusion reaction. Using internal funds, extensive plasma physics simulations have been run at LANL to explore methods to increase the neutron yield. Using a fully kinetic, 1.5 dimension plasma code with full atomic physics, we have developed an IEC design that can produce $10^{11} \mathrm{n} / \mathrm{s}$. The underlying physics principals are straight-forward. In order to increase the neutron yield, the density must be lowered below the Paschen breakdown limit. This operation can be achieved using a novel triple-grid design. (Conventional IEC systems use only a single grid). The triple-grid system decouples the plasma breakdown from the ion acceleration, thus obviating the Paschen limit.

As a parallel effort we have been investigating the gamma-detection aspect of this problem. We have been experimentally and computationally mapping out additional gamma-lines from the neutron interaction with nitrogen, including many of the cascade lines. We have also been investigating novel detector methods to increase the gamma-detection throughput. These efforts all build on existing safeguards program efforts that are already funded. In particular, we have begun work on Compton filters, detector shield designs to reduce the continuum, and the design of coincidence systems to exploit the coincidence emission of gamma rays in a cascade process. These methods have the potential of increasing the gamma ray detection rate to accommodate the increased interrogation of a high intensity source.

Finally, we have conduced MCNP simulations of these systems to scope out the system requirements. MCNP simulations have been used to explore depth and speed considerations, as well as neutron die-away times.

\section{Technical Maturity}

There are four components to this technology, the development of the IEC plasma neutron source, the development of faster gamma detectors, and the mapping out of additional gamma ray lines of interest and the MCNP scoping study.

The IEC plasma simulations have been completed to the point that the next logical step is to construct a working experiments. The plasma chamber design has been optimized using fully kinetic plasma simulations. These simulations were funded from internal laboratory funds.

The MCNP scoping studies are in progress. Initial results indicate that the $10^{11}$ source intensity is the correct value, which is confirmed by extrapolating from the INEL results with the PINS system. The MCNP study was also done using internal laboratory funds.

The mapping out of the gamma ray lines is also in progress. It is being funded from external funds for other applications. It is fortuitous that the development of neutron-gamma $(n, \gamma)$ technology has several applications in this particular case. Many of the gamma ray lines will be fully mapped out as part of other efforts.

The development of new, high-rate, gamma-ray detectors is still in the early stages of development; it is not yet funded. However, there are safeguards applications as well and we are proposing this effort in other areas at this time. 


\subsection{RESONANT MICROSTRIP PATCH ANTENNAS}

\section{Technical Contacts}

Joseph Mack

Phone: (505) 667-3416

Fax: (505) 665-3644

jmmack@lanl.gov

Robert Kelly

Phone: (505) 667-6492

Fax (505) 665-3644

rkelly@lanl.gov

\section{Description of Technology}

A continuous wave $(850 \mathrm{MHz})$ is emitted that is partly reflected and transmitted at the air-soil interface. The transmitted portion is scattered from the mine due to discontinuities in dielectric constant and conductivity. The scattered wave, along with the ground reflected wave, is sensed by the antenna, which causes a modification of both the impedance and resonant frequency. Impedance and frequency changes translate to a target presence. An approximate way to view the system is to consider the antenna, ground surface, and target as complex impedance elements in a mutually coupled tuned circuit. Note that the system is quite distinct from ground penetrating radar.

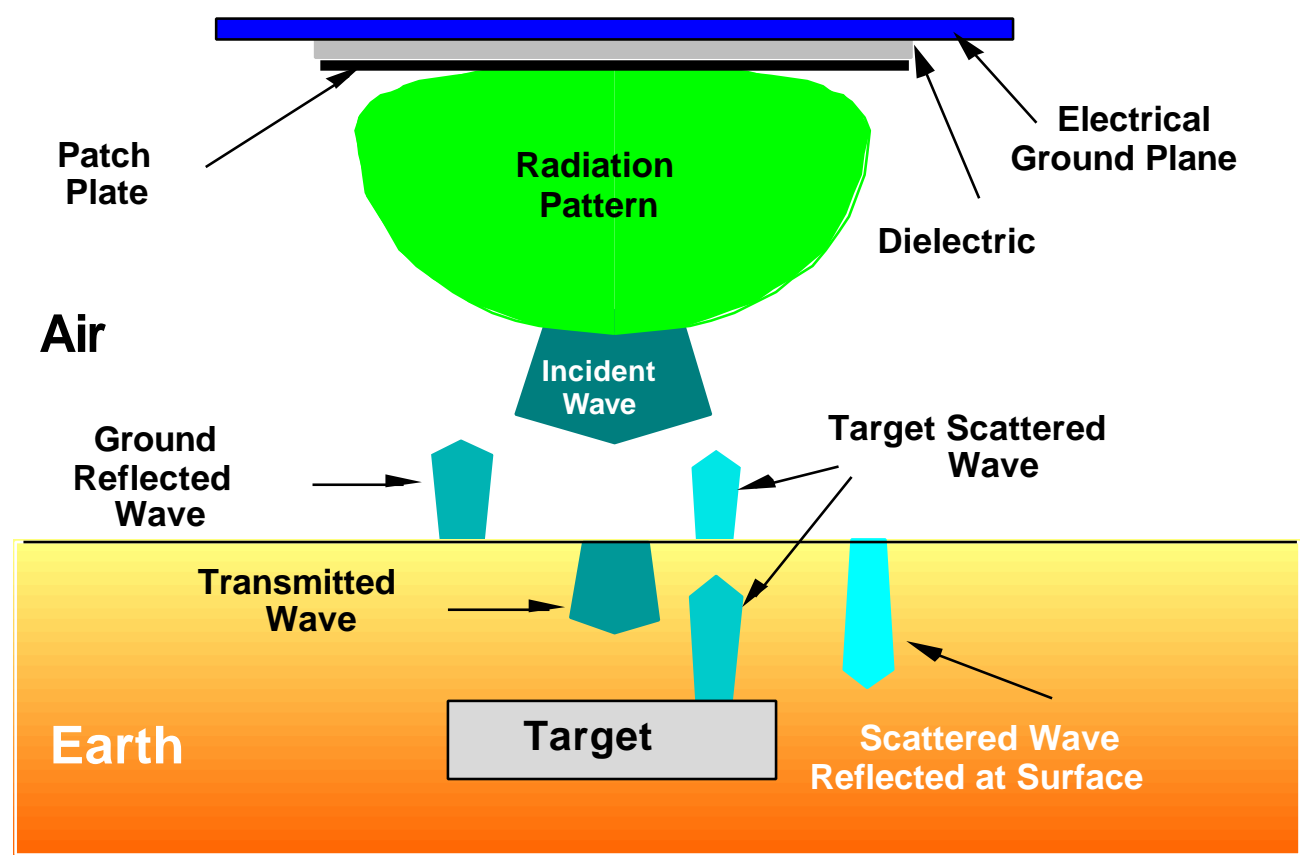

Fig. 9. Microstrip Patch Antenna. 


\section{Technical Status/Maturity}

\section{Concept}

The primary element in this landmine sensor is a microstrip patch antenna, composed of two parallel metallic plates of unequal area separated by a very thin dielectric. The smaller plate (patch) is approximately one half wavelength long. Broadside radiation occurs due to the small fringing fields at the forward and backward edges (coherent superposition of two slot radiators). It has a high Q (up to 100); therefore, a small bandwidth, and a gain of typically $7 \mathrm{~dB}$. The input impedance is governed by an equivalent parallel RCL circuit, fed by either a coaxial cable or a microstrip line. It may be viewed as a resonant cavity with electric walls for the top and bottom plates and a magnetic wall for the open sides. At resonance, the electric and magnetic energy densities are equal, and the impedance becomes purely real. The patch antennas are conformable, low-profile, mechanically robust, and possess controllable polarization.

\section{Funding}

- Internal Laboratory funding: $\sim \$ 100 \mathrm{~K}$ in FY 96

- Industrial Partner: Raton Technologies Research has invested several-hundred thousand

\section{Equipment Availability}

Mining prototype exists; reconfiguration straightforward ( 60 days)

\section{Laboratory Demonstration}

Substantial database from controlled tests

Field Demonstration

Planned (pending external funding)

\section{Applications/Testing}

- can sense and image small (or large) AP metal/non-metal mines

- extensive clutter tests performed and documented

- appears to be a robust, low-cost, powerful anomaly sensor

\section{Further development}

(a) Development of a patch-array prototype, using an optimal combination of time and phase scanning (and possibly frequency diversity), to enhance and quickly form the target images;

(b) Further investigation of soil properties, height and depth sensitivities, more clutter;

(c) Blind test concept demonstration; and

(d) Formulation of engineering development and implementation plans. 


\title{
6.4 COMPLETELY PASSIVE LOCATION AND CHARACTERIZATION OF LAND MINES WITH SUPERCONDUCTING QUANTUM INTERFERENCE DEVICE (SQUID) SENSORS
}

\author{
Technical Contact
}

Dr. Robert H. Kraus, Jr.

Los Alamos National Laboratory

P-21, MS-D454

Los Alamos, NM 87545

Phone: (505) 665-1938

Fax: (505) 665-4507

E-mail: rkraus@lanl.gov

\section{Description of Technology}

The focus of this effort is to use completely passive techniques for locating and characterizing buried land mines, associated hardware such as booby traps, and clutter. This effort will be to use Superconducting Quantum Interference Device (SQUID) sensors, the most sensitive magnetic sensor known, to passively locate and characterize ("image") land mines and the immediate area around the mine. This effort is divided into three phases, each successive phase dependent on the success of the preceding phase and the needs of the customer. Phase 1 will culminate in a laboratory demonstration of the sensor capability to localize a land mine-like target in a realistic environment. Phases 2 will develop a fieldable sensor array prototype necessary to demonstrate land mine characterization and clutter isolation in a field test. Phase 3 will integrate the electromagnetic inverse solver software necessary to process sensor array data for real time threat and clutter assessment. Phases 2 and 3 can be accomplished in parallel if necessary advanced planning and funding are provided. This sensor system can readily be extended to include active (induction) land mine detection methodologies by adding induction coils to the sensor array once it is completed. This would give the user an integrated land mine sensor system with the option of using either passive, active, or both techniques (via computer control).

Mine detection hardware that uses induction methods with fairly low-sensitivity sensors are becoming less reliable with the increased use of mines that contain very small quantities of metal, or use triggers that are activated by the induction field (such as suspended permanent magnet triggers). These methods lack the sensitivity needed to detect land mines containing small amounts of metal (or other conducting materials) and produce sufficiently large electromagnetic fields that certain classes of land mines will be triggered by the induction field of the detector. Sensors sufficiently sensitive to detect land mines containing only small amounts of metal will also detect all of the clutter in the field resulting from man made debris (i.e. metallic garbage or shrapnel) or geologic deposits. Traditional land mine detectors do not have the capability to accurately differentiate between clutter and a real mine. Thus, all suspect items must be painstakingly interrogated by hand. Ultimately, a reliable and efficient land mine sensor will be a fusion of multiple sensor technologies that address different aspects of the problem. Among the most important sensor technologies are high-sensitivity passive magnetic detection and electromagnetic induction techniques.

SQUID sensors were identified as the best available technology to locate unexploded ordinance in a heavily cluttered environment by a blue ribbon panel convened under a US Army contract by NASA JPL (Sensor Technology Assessment for Ordinance and Explosive Waste Detection and Location, 1 March 1995, pub. JPL D-11367, rev B). The greatest impediment to field deployment of SQUID technology has been the requirement of liquid helium ( $\mathrm{LHe}$ ) and the associated severe cryogenic issues associated with LHe. Current high-critical temperature (HTC) SQUID technology has been advanced sufficiently to dramatically simplify this constraint. HTC SQUIDs use liquid nitrogen (LN) or even cryogen-free battery operated cryocoolers. 
LN can readily be transported and handled with the same degree of concern as a hot cup of coffee, and a few liters is sufficient to cool SQUID sensor array for several days.

\section{Concept}

The approach proposed here is to adapt technology used to image magnetic fields in the human body (most commonly from the brain or heart) to the problem of locating land mines in a cluttered environment. Although the technology is complex, much of the development of sensor systems and analysis paradigms and algorithms accomplished in support of the brain imaging program at Los Alamos can be directly adapted to the land mine problem.

The key technical accomplishments foreseen in this effort to field-deploy an effective SQUID sensor system are 1) building HTC SQUID sensor systems, 2) building portable electronics and data acquisition and analysis systems, 3) improving noise rejection, and 4) adapting existing biomagnetic imaging software to the land mine problem. Parts of items 1-3 are already being addressed as parts of other ongoing efforts by our group. The first sensor system will be a relatively simple system to validate the concept and provide for an early demonstration. Upon completion of the initial demonstration, a sensor array will be built for area field mapping and field imaging to localize and characterize land mines in a cluttered environment. Sensor array data is needed for reliable land mine discrimination from clutter. Finally, although one could conceivable locate mines by eye from field maps, it is far more efficient to implement a computer algorithm to analyze the field maps and search for land mine signatures. Although not included in this proposal, which focuses on developing a passive magnetic land mine detection capability, an induction coil can be added to the sensor array and with only modest additions to the sensor instrumentation and analysis software, a single system will provide a fusion of passive and active electromagnetic sensors.

\section{Initial Phase}

1. Understand specific needs and interests of the customer.

2. Develop SQUID-based sensor system for initial capability demonstration.

3. Validate sensor capability in controlled environment with realistic mine models.

\section{Final Phase}

1. Develop electromagnetic inverse model for cluttered environment.

2. Implement electromagnetic inverse solver for localization and characterization of various mines and associated components in cluttered field environment.

3. Demonstrate real-time performance of mine sensor with analysis software.

\section{Funding}

None, at present.

\subsection{ULTRASENSITIVE MINIATURIZED SENSOR SYSTEM FOR DETECTION OF UXO}

\section{Technical Contact}

Basil I. Swanson

Los Alamos National Laboratory

Chemical Science and Technology Division 
Phone: (505) 667-5814

E-mail: basil@lanl.gov

\section{Description of Technology}

Rugged, portable, and miniaturized sensor systems for the real-time point detection and identification of unexploded ordnance (UXO) are evolving from ongoing Laboratory research efforts. Very recent breakthroughs in this area indicate that sensor sensitivities in the parts-per-quadrillion range are achievable.

These microsensors are based on species selective thin-film technology developed over the past few years for real-time sensing of volatile organic compounds (VOCs) and chemical warfare agents. This effort has resulted in three patents, several publications and presentations at national meetings, and an R\&D 100 award for reversible real-time monitoring of VOCs. Within the near term, this technology, coupled with advanced data-processing techniques and systems design and engineering, can be integrated into a miniaturized, simple, and reliable sensor system for a variety of military and civilian applications. This system would have highsensitivity, continuous and autonomous operating modes, and provide positive identification and quantification of low volatility signature chemicals for explosives while eliminating false positives from background interferants. In addition to their use in detecting UXO associated with, for example, buried land mines, longer-term advances could lead to the development of species selective microsensors capable of detecting sea mines. Miniaturized smart sensor systems based on sensor arrays could also be adapted to robots to a) provide chemical sensor capabilities for remote UXO detection and b) provide feedback to help guide robotics activities

The microsensor system is based on chemical recognition and novel surface modification techniques (molecular "self-assembly") that allow the covalent attachment of species-selective host reagents to the surface of a sensing transducer. The reagents are "bucket" like molecules that can be deposited as thin, densely packed films to allow the capture of specific chemical compounds. These host reagents form inclusion complexes with target molecules where the molecule to be sensed fits inside the cavity of the host reagent. Because the formation of inclusion complexes is reversible, these reagents can be used to fabricate near real-time sensors. The sensitivity can be tuned by varying the size of the host reagent cavity and by optimizing the chemical functionality of the rims of the molecular buckets. Through the combination of selfassembled multilayer films, chemically-tuned reagents, and simple surface-acoustic-wave transducers, microsensors arrays have been fabricated that distinguish between many different organics in the 10-ppm to 100-ppb range.

Recent advances in optimizing the binding affinities of the host reagent molecules to nitroaromatics and nerve and mustard simulants have demonstrated low ppb sensitivity for monolayer films. Associated with the significant enhancement in sensitivity of this film to nitrobenzene and nerve and mustard simulants is a corresponding enhancement in selectivity, as demonstrated by the observed 3-4 orders of magnitude decrease in response to VOCs and other interferants. We expect that an additional 1-2 orders of magnitude improvement in sensitivity can be achieved in further modification of the chemical functionality at the rims of the host reagents, and another 1-2 orders of magnitude improvement is possible by forming multilayer films. Accordingly, without any additional optimization of the simple SAW (surface acoustic wave) transduction techniques now employed, we expect sensitivities in the low ppt range. Further improvements in sensitivity can be made using either advanced optical transducers or acoustic resonators that operate at very-high frequency.

Through the use of highly sensitive and selective host reagents, multilayer technologies, and advanced transduction technologies it will be possible to achieve parts-per-quadrillion sensitivities with little interference from common volatile species and contaminants. Miniaturization of the $\mathrm{CW}$-sensor package can be 
accomplished through integration of the sensor elements and the front-end electronics onto a multichip module, using the advanced optical, electronic, and signal-processing technologies being developed within the Laboratories space-satellite programs.

\subsection{UXO AND LAND MINE DETECTION USING CHEMICAL MICROSENSORS BASED ON SPECIES SELECTIVE FILMS}

\section{Technical Contact}

Basil I. Swanson

Los Alamos National laboratory

CST-1, MS J565

Los Alamos, New Mexico, 87545

Phone: (505) 667-5814

E-mail: basil@lanl.gov

\section{R\&D Objectives}

Develop a sensor array system based on species selective films and optical heterodyne detection capable of near-real-time detection of antipersonnel land mines and unexploded ordnance. Test this sensor array

system against explosives vapors generated by a calibrated explosives delivery system and transition to field tests of land mines and UXO.

\section{Technical Approach}

The technical approach is based on a) a smart sensor array system with built-in pattern recognition, b) species selective thin films that provide high sensitivities AND specificity, c) optical heterodyne detection for optimum sensitivity in transduction, d) systems integration and miniaturization for flexibility in deployment, and e) tests against real CW agents and interferents at a DOD lab. A brief explanation and rationale for these four critical elements is presented below along with a status report and projections of expected systems attributes.

\section{Sensor Array System}

The sensor system concept under development is based on an array of sensor elements each of which has a different species selective coating bonded to the transducer surface. In effect, this approach mimics the olfactory system of a dog's nose in that each sensor element (receptor in the case of the olfactory system) is capable of "sensing" multiple analytes. It is through pattern recognition of a array of these distinct sensor elements that the identity and amount of the chemical species present in a complex odor can be measured. Because it is not yet possible to mimic olfactory signal amplification, the species selective films utilized here are designed to optimize both sensitivity to the target $\mathrm{CW}$ agents while also minimizing the response to interferents. We envision an array based on roughly a dozen distinct sensing elements including reference elements. The system will also include a pattern recognition algorithm that provides a simple answer in terms of exposure based on the complex response of the sensor array. 


\section{Species Selective Films}

Species selective thin films based on guest-host complex formation and self-assembly are under development for near real time sensing of $\mathrm{CW}$ agents and explosives. Using surface acoustic wave (SAW) devices we have been able to achieve low to sub parts-per-billion ( $\mathrm{ppb}$ ) sensitivities for selected nitroaromatics. A delivery system for selected explosives in under development and measurements for TNT and DNT will be initiated shortly. Current results demonstrate that these films exhibit high specificity with the response against common interferents being 3-4 orders of magnitude less than that to nitroaromatics. The species selective reagents will be further tailored to enhance the sensitivity AND specificities to TNT and DNT. The species selective thin films are based on cavity shaped molecules that can be tailored to optimize the binding affinities to explosives. Specifically, cyclodextrin molecules will be functionalized to increase non-covalent interactions with explosives. The CD reagents are covalently attached to functionalized self-assembled monolayers that are themselves covalently attached to optical and SAW transducers. Monolayer films and multilayer films that are based on the growth of ultra-thin polymer films that have CD reagents covalently attached to the polymer backbone are being studied. The further development of these films will be guided by results obtained from lab measurements at Los Alamos against explosives vapors. We expect 1-2 orders of magnitude improvement in the sensitivities of these films to explosives vapors by specific tailoring of the CD host reagents.

\section{Optical Heterodyne Detection}

Our current project with DARPA in land mine detection involves a collaboration with Rockwell Science Center who is responsible for the development of ultrasensitive thin film resonators (TFRs). The expectation is that TFRs will be capable of 2-3 orders of magnitude improvement relative to SAW devices. We have also begun to independently develop optical heterodyne transduction techniques to enhance signal response and have recently achieved the same sensitivity as that obtained using SAW devices (below). This preliminary work has been based on polarimetric optical detection using a Zeeman laser based interferometer. In collaboration with N. Peyghambarian of the University of Arizona, we will further develop optical heterodyne detection techniques and, in particular, polarimeteric methods as well as distributed Bragg reflectors (DBR). Side-by-side comparisons of the monolithic DBR, polarimetric and SAW transducers will be performed to benchmark transduction development. We expect to be able to enhance sensitivity by 2-3 orders of magnitude with improvements in optical transduction. Combined with the expected 1-2 orders of magnitude improvement with improved recognition elements, we project ultimate sensitivities in the sub-ppt (parts per trillion) range.

\section{System Integration and Miniaturization}

The target of our current work is a laboratory based prototype capable of measurements at a DOD lab as well as future field measurements. Nonetheless, the ultimate target is a miniaturized, cost effective smart sensor array system that can be deployed in a variety of ways. The intent is to develop a sensor array system where each element is integrated with the driver electronics, pattern recognition algorithm, and detection onto a single multichip module (MCM). This MCM is then integrated into a sensor module to permit real-time gas sampling and necessary environmental controls. The critical aspect of this goal is to be able to fabricate a miniaturized multi-element sensor array with distinct films on each element. The individual transducer elements (either waveguides or DBRs) are extremely small and the art will be in patterning each element with different films.

\section{Sensor Measurements}

Measurements of SAW and optical transducers against explosives vapors and interferents will be performed at the sensor test-bed under development at Los Alamos. In view of the extreme "stickiness" of the target 
explosives vapors, the development of calibrated sources capable of delivering varying amounts of explosives vapors is not trivial. We are working with John Parmeter of SNL to co-develop a suitable delivery system that couples his diffusion generator with our passivation films that minimize adsorption of explosives vapors on walls.

\section{Collaborations}

The work described herein is collaborative with Karen Grace (Nonproliferation and International Security, Los Alamos) and several external organizations. John Parmeter, of Sandia National laboratory, has a long standing interest in explosives detection and is helping with the development of calibrated gas delivery systems for explosives. Nasser Peyghambarian and colleagues of the Optical Science Center at the University of Arizona provide expertise in optical transduction and waveguides. Rosemary Smith of UC Davis provides expertise in microfabrication and integrated optics. 


\section{OAK RIDGE NATIONAL LABORATORY}

\subsection{BIOREPORTER BACTERIA FOR LANDMINE DETECTION}

\section{Technical Contact}

Robert S. Burlage

Environmental Sciences Division

POB 2008, MS-6036

Oak Ridge National Laboratory

Oak Ridge TN 37831

Phone: (865) 574-7321

Fax: (865) 576-8543

E-mail: RVX@ORNL.GOV

for US EPA.

\section{Description of Technology}

Old mine fields remain active, endangering non-combatants long after the war is over. Identifying the location of these mines is a difficult and dangerous occupation. The mines have few properties that make them stand out from the soil in which they are placed. However, old landmines (and other UXO) gradually leak small concentrations of their explosives into the soil. Vapors from these explosives have been detected by dogs or by chemical "sniffers", although in the latter case the response time is unacceptable slow.

Explosive chemicals can be detected by bacteria, which have adapted to scavenge low concentrations of nutrients. Uptake of these chemicals results in the triggering of specific genes in the bacteria. We are creating bioreporter bacteria that detect the location of leaking mines by scavenging the small amounts of explosives around a mine. These bacteria will be genetically engineered to produce a brightly fluorescent compound in response to the presence of specific explosives. By finding the fluorescent bacteria, you find the landmine. Techniques for application of the bacteria to the minefield and for detection are described.

One of the critical aspects of this system is its response time. The system has two parts: application and sensing. During the application phase the bacteria are sprayed over a large area and allowed to contact the small concentrations of explosives in the soil. During the sensing phase the minefield is illuminated with ultraviolet light and the fluorescent "hotspots" are identified. Application can be time-consuming, although vast areas can be prepared for detection. The sensing phase is technically simple, and can be carried out rapidly (i.e. at slow driving speed). 


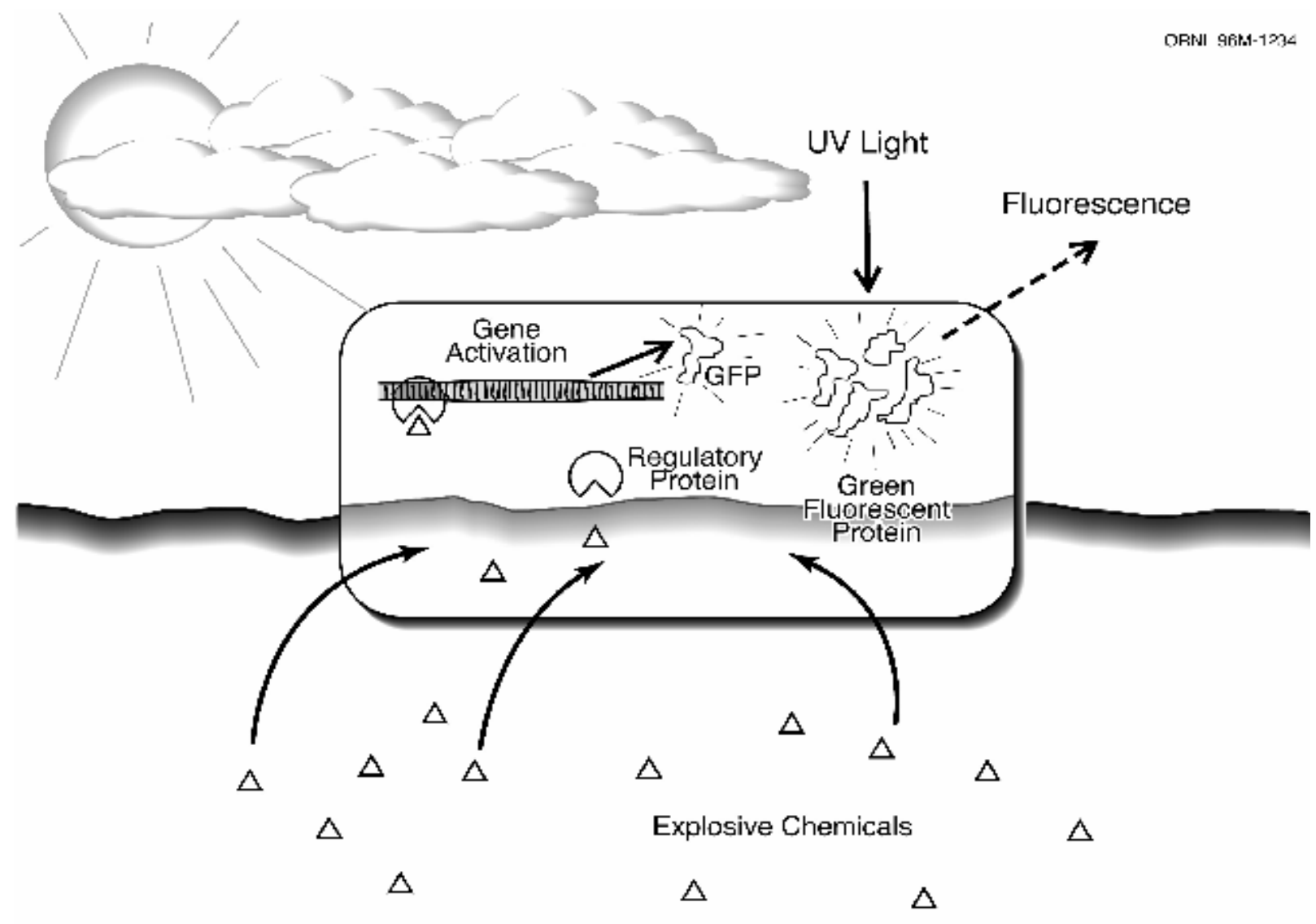

Fig. 10 Bioreporter Bacteria Operation.

The central feature of this technology is the creation of a genetically recombinant bacterial strain that scavenges specific explosive chemicals, and which then responds by producing large amounts of a fluorescent protein. Bacteria which degrade explosives have been studied extensively, and genetic techniques to modify the strains are available. The expression of these degradation genes is controlled by a regulatory protein that also recognizes the explosive chemical; only in the presence of the chemical will the regulatory protein activate the degradation genes.

A genetic fusion between these genes and a bioreporter gene are being constructed. The gene for Green Fluorescent Protein (GFP) is being used because it results in a protein of extremely bright fluorescence. This fusion can be produced using a special vector developed in this laboratory. A benefit of using this special vector is that an induced gene will remain permanently induced, allowing production of large amounts of GFP. Overproduction of GFP will make the cells easier to detect. 


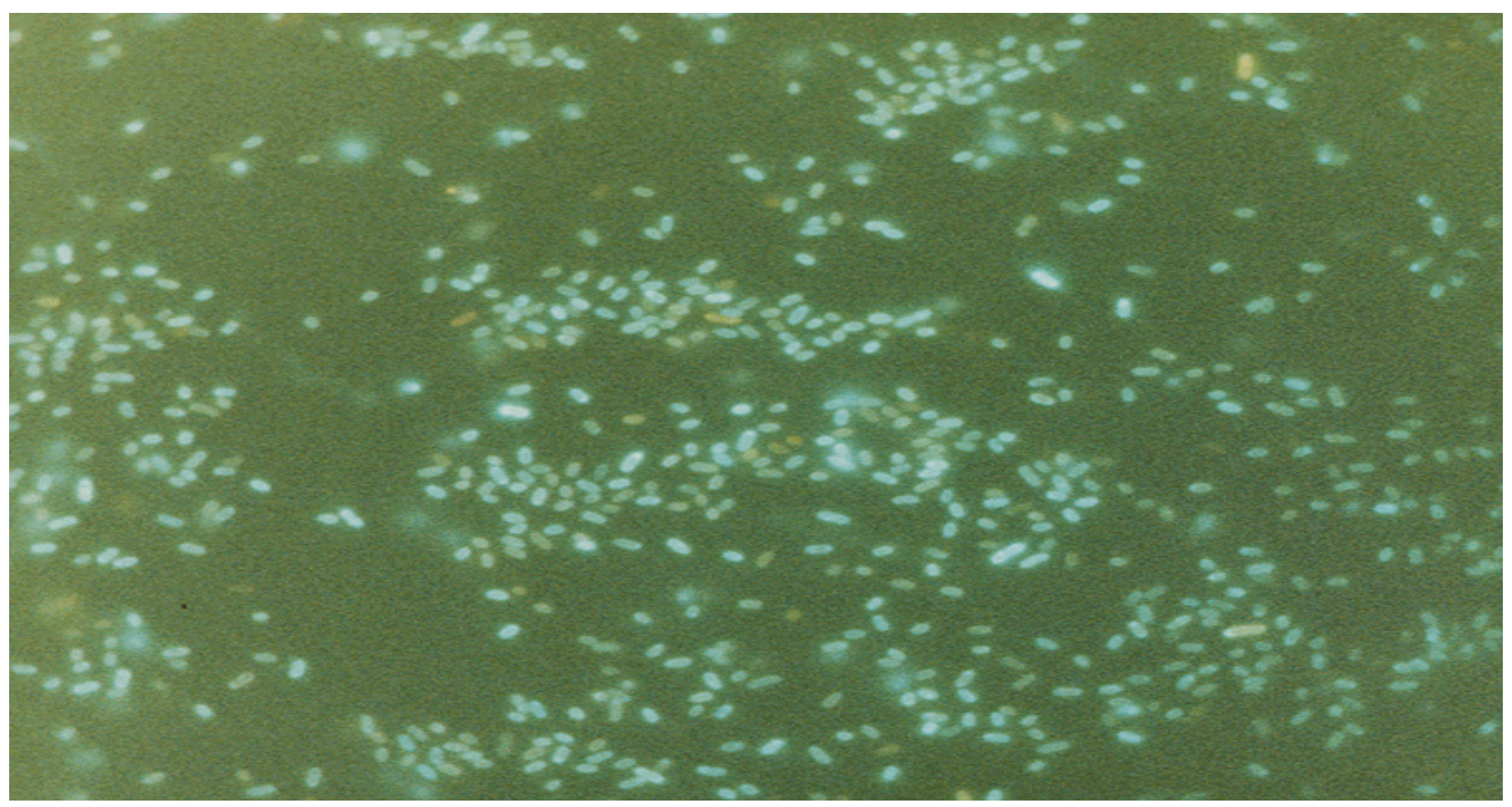

Fig. 11. Bioreporter Bacteria.

ORNL has extensive experience in working with bioreporter bacteria for the examination of bacterial gene expression. These studies concentrate on bacteria for the remediation of hazardous waste, and ORNL has published on bacteria strains for detection of hydrocarbons (e.g. toluene, naphthalene), mercury (both organic and inorganic), and a variety of exotic bioreporters of environmental conditions (e.g. nutrient starvation, DNA damaging events). It is this experience that makes ORNL confident of the ability of bacteria to detect small concentrations of specific chemicals. For instance, published results for mercury show detectability at a subnanomolar concentration, while detectable concentrations of naphthalene in the part-per-million range are routine. These reports made use of the bioluminescent lux system; the relatively new fluorescent system has the potentially for increases of sensitivity by several orders of magnitude. In addition to greater sensitivity, the fluorescent bioreporter system produces a stable fluorescent molecule that can be detected for days after it is made. We use these bacteria as tracers for groundwater movement studies, where they have produced excellent results.

The bacteria can be applied to the field by spraying, such as with a hand sprayer or over large areas with a crop duster. After a wait of several hours for the bacteria to work, the fluorescent "hotspots" can then be detected on a dark night using a hand-held or truck-mounted UV light.

It is possible that other detection methods for microorganisms might be created, such as a colorimetric bacterial strain that could be seen easily in the daylight. However, this strain would require substantial development in the laboratory, and for the proof-of-principle experiment it is highly recommended that a proven system (such as the fluorescent bacteria) be used. It is possible that conventional military equipment might be easily adapted for detection of the fluorescent hotspots, although the UV lamp and detection apparatus represent off-the-shelf technology, and are not expensive. 


\section{Deployment}

The deployment of this invention in the field requires two steps, (1) application and (2) detection. The first step is equivalent to equipment preparation time, and is not a component of the detection speed.

(1) During the application phase the bioreporter bacteria are applied to a large area that contains landmines in unknown locations. This is easily performed using conventional spraying technology, such as with a handheld garden sprayer over small areas, a hose for intermediate size areas, or a crop duster over large (100's of acres) areas. The bacteria survive well under these application conditions.

Application is followed by an incubation period of hours while the bacteria contact the explosives and produce the fluorescent bioreporter protein. It is notable that this time period is constant and independent of the size of the area to be examined. Therefore, it is more economical to examine larger areas. As the area to be examined increases, the cost per detected mine decreases.

ORNL-DWG 96M-1235
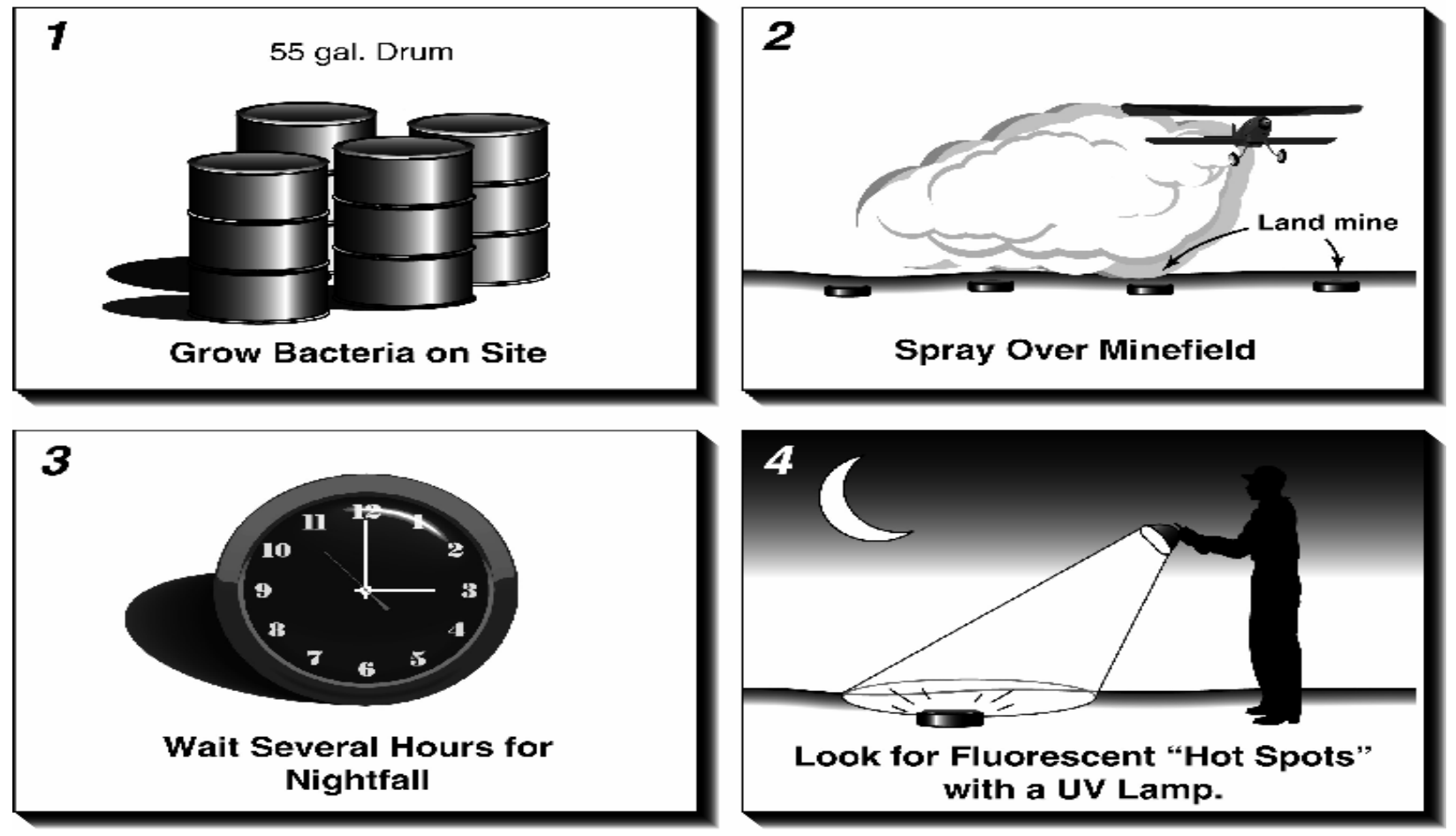

Fig. 12. Bioreporter Bacteria Methodology.

It is also noteworthy that this phase is accomplished without undue risk to the personnel involved i.e. entry into the minefield area is not required.

(2) During the incubation period the bacteria have produced a fluorescent signal at the location of the explosives. In the detection phase the minefield is examined for the presence of the fluorescent signal. This activity can be easily performed with a conventional ultraviolet light and a technician who can visually identify the fluorescent spots and mark them appropriately. This activity can also be performed with fluorescent detectors that are robot-propelled and which electronically label the fluorescent spots. The detector capability is also conventional, relying on a photodetector for the identification of a fluorescent spot. More sophisticated detectors may be able to perform their task in the daylight, by shielding the area under examination from 
daylight. Increased fluorescent detector sensitivity would result in more mines detected, particularly those mines that are leaking only trace amounts of explosives. In each case the technology requires little or no development, is inexpensive and easy to operate. However, for the proof-of-principle experiment only the conventional UV light and technician will be used.

This activity can be performed very rapidly when compared with conventional landmine detection technology. For instance, the technician can examine an area at walking speed $(3 \mathrm{mph})$ while sweeping an area of 4-5 feet wide. A vehicle mounted detector can hold a boom of $10 \mathrm{ft}$. width, and travel at a greater speed. Exactly how fast the vehicle can travel is dependent on the landmine density (i.e. how often must the vehicle stop) and the speed at which the detector operates (fluorescent detectors are available that report the signal almost instantaneously). Although outside the scope of the present proposal, it is possible that an entire minefield could be briefly illuminated and the fluorescent areas mapped instantaneously from an airborne detector.

\section{Strengths of the Technology}

\section{Sensitivity}

Detectors have been developed which "sniff' the air above the ground for explosive vapors. These techniques may be unsuitable for the field because of sensitivity (vapors are present at extremely low concentrations), because of slight winds that disturb the sample area, or because of long reaction times (the detector should give a result in seconds). Nevertheless, it is clear from previous studies that the vapors are present at a detectable concentration, and therefore the explosive chemicals are also in the soil proximal to the landmine. The bacteria that are used in this technique detect the explosive chemicals in the soil, not in the vapor phase. Concentrations in the soil may be much greater than in the vapor phase, and are not subject to shifting winds. In addition, the genetic construction forces the bacteria to respond to the presence of the explosive chemical by creating a strong fluorescent signal, in effect amplifying the chemical signal. This amplification of the signal makes detection instantaneous, because the fluorescent spot will be easily visible to the technician.

\section{Portability}

The detector in this technique is the bacterial strain, which can be carried virtually anywhere and grown to field-scale volumes using common microbiological practices. Growth media are typically dried powders; site water can be purified to make a batch fermentation. The required equipment and power supply are probably available at a typical field kitchen. Application and detection equipment, as described above, represent off-theshelf technology and are neither expensive nor cumbersome, and are easy to operate.

A rough calculation shows that $10 \mathrm{E} 3$ bacteria per $\mathrm{cmE} 2$ are needed for a visible signal. This equates to $4 \mathrm{X}$ 10E10 bacteria per acre or 10E11 per hectare. Standard fermentation yields about 10E11 bacteria per liter (i.e. enough to cover a hectare) while a 55 gallon drum yields enough bacteria to cover 500 acres (i.e. a large farm).

\section{Weaknesses of the Technology}

\section{Response time}

The fluorescent bacteria require several hours for the uptake of the leaking explosives, expression of the bioreporter genes, and formation of the fluorescent protein. There is little that can be done to accelerate this process without substantial research input. With the demonstration of this system's value, it is hoped that developments can be gradually introduced to increase the system's versatility. However, as explained above, 
the time required for the application phase is not fundamentally different from preparation times for other techniques.

\section{Field conditions}

The bacteria must contact the explosives in the soil in order for a response to be generated. Conditions that prevent soil contact (ice, snow, very heavy vegetation) should be avoided. A downpour of rain would tend to wash the bacteria away, although a light drizzle would probably not be an obstacle, and might even improve detection.

Safety issues

Large-scale application of bacteria is no longer an uncommon practice. For instance, artificial snowmaking utilizes a bacterium that allows efficient snow crystallization to occur, and is used at many ski resorts. The use of a genetically recombinant strain is a rare event outside of the laboratory, and a procedure for evaluating proposed releases has been established by U.S. EPA. We have recently been successful in obtaining permission for field work with one of our recombinant strains, and believe that this experience will make permission for subsequent field releases easier to obtain. The microorganisms that we have identified for this technique are not pathogens, and protocols for the safe handling and application of the bacteria will be clearly established. As experience with application of the bacteria increases, resistance to its use will decrease.

\section{Funding}

Internal - Laboratory Director's Research and Development (LDRD)

\section{Status/Maturity}

Bacteria which feed upon a variety of explosive compounds are being evaluated for genetic engineering.

\subsection{QUADRUPOLE RESONANCE FOR MINE DETECTION}

\section{Technical Contact}

Steven W. Kercel

Oak Ridge National Laboratory

Instrumentation and Controls Division

Phone: (865) 574-5278

E-mail: kercelsw@ornl.gov

\section{Description of Technology}

Notwithstanding the work performed by many organizations, there has been no significant breakthrough in electronic mine detection since the Korean war. Due to the wide variety of mine types, soils, and hard realities of the mine detection problem, many "high-tech" approaches have proven unsatisfactory. The situation poses two questions. Is there something that all mines have common that distinguishes them from most non-mine objects? Obviously they all contain explosives, and despite the fact that there are hundreds of mine types, there are only a few explosive types. Is there an as yet untried technology that might prove feasible? Detection of quadrupole resonance signatures of the explosive material may be feasible for realworld mine detection. 


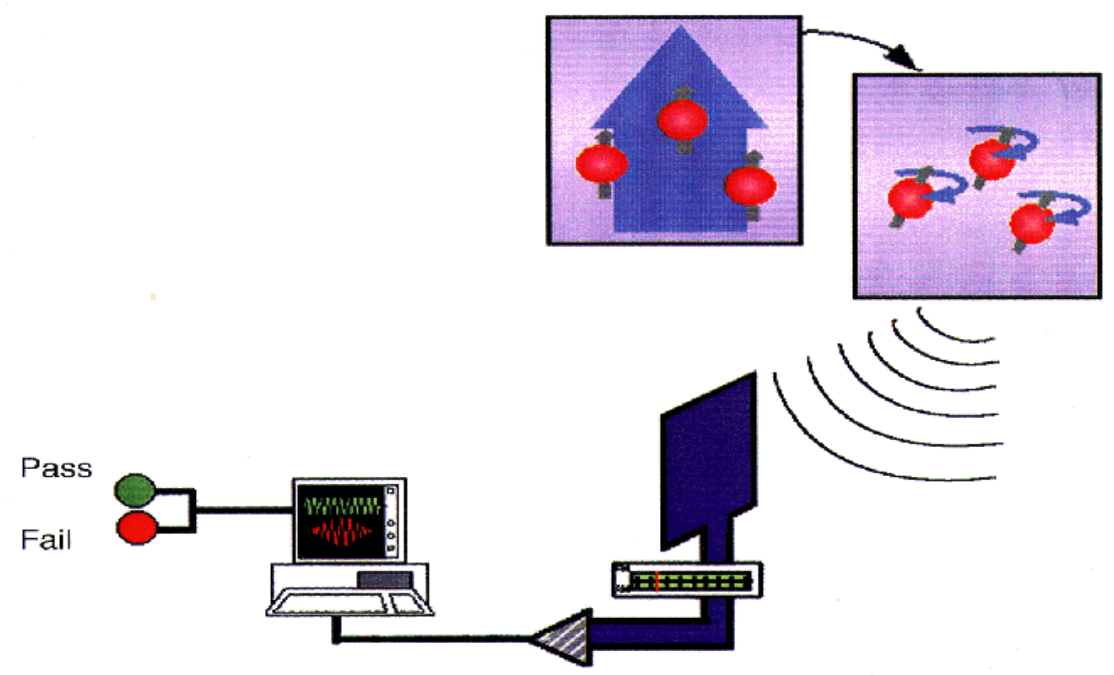

Fig. 13. Quadrupole Resonance System.

As already noted, there are relatively few explosive compounds used in mines. They are typically crystalline solids containing nitrogen. Due to quadrupole resonances of the nitrogen nucleus trapped in the structure of an explosive molecule, explosive compounds radiate distinct and well documented signatures in response to radio frequency $(\mathrm{RF})$ excitation in the region of $40-8000 \mathrm{kHz}$. The signature comes from the nucleus of the atom as constrained by the molecular structure. Thus, the signature of the nitrogen in potassium nitrate is an attribute of the potassium nitrate, not the elemental nitrogen.

Compared to conventional explosives detectors, quadrupole resonance has several advantages. Since the mechanism depends on an electromagnetic response to an electromagnetic stimulus, the sample can be several inches from the coupling coils and still be perceived. The problems that plague conventional chemical analyzers, low (or non-existent) vapor pressure, and coupling the system to the sample, are not present with quadrupole resonance. Quadrupole resonance signatures of explosive compounds are quite distinct from signatures of elemental nitrogen or non-explosive nitrogen compounds. Thus, quadrupole resonance systems are not as vulnerable to false positives as neutron-based systems. Also, quadrupole resonance systems do not require the powerful magnetic fields that are needed by nuclear magnetic resonance systems.

If quadrupole resonance is so good, why isn't everybody doing it? The short answer is that it is a very weak effect. The distinctive signatures are there, but the signal to noise ratio is so poor that the signatures are difficult (but not fundamentally impossible) to pull out of the noise. Conventional physics experiments overcome the problem by using a powerful RF source (on the order of megaWatts) for the excitation. This approach is good for physics experiments but not much else.

Nevertheless, the Federal Aviation Administration has sponsored the Naval Research Laboratory in research into using quadrupole resonance for the detection of explosives hidden in airline baggage, and the results are encouraging. The research finds that the development of a commercial device to detect RDX and PETN in airline luggage is feasible and should proceed. There is also no fundamental reason that TNT, AN, AP, $\mathrm{KNO}_{3}$, and NC could not also be detected. Furthermore, it should be feasible to build 
a detector that can discern a mixture of explosives. NRL researchers have patented several coil designs that are intended to be used for quadrupole-based airline baggage detection.

What does it work now when it didn't in the past? As technology advances, the ability to inexpensively extract useful information from a weak signature improves. The cliche has it that "a dB is a dB is a dB", and that no mater where it appears in the system, any $\mathrm{dB}$ of improvement is just as good as any other. The SQUID magnetometer provides a broadband low-noise means to put the $\mathrm{dB}$ improvement on the receiving end instead of the transmitting end. The emergence of high temperature superconductors has made it possible to have a field deployable SQUID that uses conventional refrigeration, and does not require liquid helium. In a SQUID-based quadrupole resonance system, a 400 Watt RF source will suffice. Beyond this, recently discovered signal processing techniques can improve the effective signal to noise ratio even further.

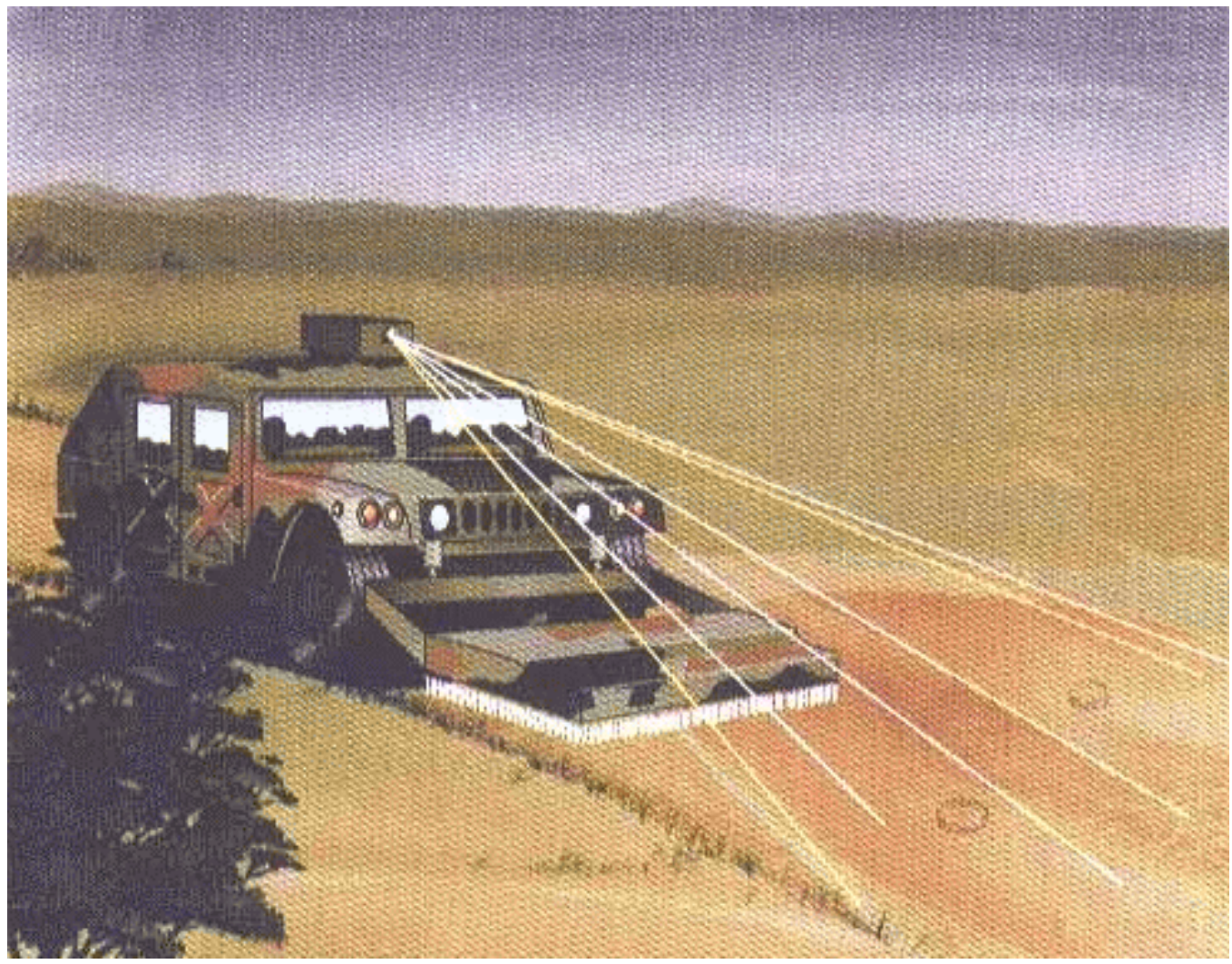

Fig. 14. Concept of Military V System NQR.

As a mine detection system, quadrupole resonance looks practical only for on-vehicle use. The need for a SQUID and a 400-Watt transmitter precludes the use of this technology in a man-portable system. The inherent weakness of the signature, and the consequent need to have the coils within a few inches of the sample, preclude its use in airplane-based systems. However, while a 400-Watt 5-MHz transmitter and a high temperature SQUID are inconvenient, they will fit into a vehicle. The coils can be sized such that they could be conveniently mounted on a fixture a few feet in front of the wheels of the vehicle. The signal processing requirements are feasible to do in real time with a set of fewer than 10 parallel DSP chips. Thus, the idea of an on-vehicle quadrupole resonance based mine detector, operating in real time, producing virtually no false negatives, and very few false positives, and generating a reading fast enough that the vehicle could move forward at 3-5 mph, is not fundamentally impractical and should be investigated. 
There are several special skills that ORNL can bring to bear on this development. First, as demonstrated in the Enclosed Space Detection System, it is practical to use one-dimensional wavelet-based techniques to identify the presence of extremely weak signals buried in sensor noise. Second, as demonstrated in the Magnetic Spectral Receiver, this can be done at frequencies up to 5-MHz on dedicated DSP hardware, and deployed in a harsh environment. Third, in the Fusion Energy program, ORNL became recognized for world-class developments of electromagnetic coupling devices that operate in the induction-field, and the near-field. Fourth, ready access to a SQUID magnetometer should not be a problem.

Low sensitivity is the major technical risk of trying to develop a mine detector based on quadrupole resonance. NRL researchers report success at detecting sub-kilogram quantities of explosive. In a practical mine detector, it would be necessary to detect quantities on the order of grams. ORNL's advanced signal processing methods could provide the margin of success in addressing this problem.

\section{Funding}

Internal - Laboratory Director's Research and Development

\section{Status/Maturity}

This technology has been commercialized for detection of explosives in airline baggage. Addition research is necessary to develop this technology to detect landmines and unexploded ordnance in the field.

\subsection{TRACE CHEMICAL DETECTION USING DIRECT SAMPLING ION TRAP MASS SPECTROMETRY}

\section{Technical Contact}

Stephen A. Lammert, Ph.D.

Chemical and Analytical Sciences Division

Oak Ridge National Laboratory

MS-6120; Bldg 4500S

P.O. Box 2008, Oak Ridge, TN 37831-6120

Phone: (865) 574-4879

Fax: (865) 576-7956

E-mail: lammertsa@ornl.gov

\section{Description of Technology}

The Chemical and Analytical Sciences Division (CASD) at Oak Ridge National Laboratory (ORNL) is a leader in the development of ion traps as analytical mass spectrometers. Within the CASD, there are currently over 20 investigators working on both fundamental and applied aspects of ion trap mass spectrometry on more than 30 ion trap based instruments. A significant emphasis within the division has been placed on the development of field-portable instrumentation. This area includes all aspects from the original research concept and validation all the way to deployment of the final instrument. Examples of fundamental research areas include: novel ion trap scan modes, theoretical models for resolution, collisional activation and kinetic processes, buffer gas effects, high mass and high resolution operation, and electrospray fundamentals. In the area of instrument development, both ground up and modified commercial instruments have been constructed for a variety of sponsors (DOE, DOD, FAA, Commerce) and applications including environmental, military, treaty support, threat monitoring (explosives, illicit drugs, and chemical weapons) and process monitoring. 
Examples of instrumental capabilities developed by groups within CASD including direct sampling introduction, atmospheric sampling glow discharge ionization for explosives detection and generic, easy-touse ion trap software. ORNL-developed instruments have undergone extensive field testing and validation as is evidenced by participation in over 20 field demonstration activities in the last 3 years. CASD-developed instruments have been used or are currently employed by DOD operations at the Rocky Mountain Arsenal (Denver, CO), Waterways Experiment Station (Vicksburg, MS) and Tooele Army Depot (Tooele, UT).

Although chemical sensing of buried mines has been evaluated in the past and found to be of little utility, advances in analytical detection technology (especially mass spectrometry) suggest that this technology should be reevaluated. For example, the development the ion trap MS in 1983 set new standards for MS sensitivity. Further ion trap developments (MS/MS in 1985, chemical ionization techniques in 1990, advanced waveform ion manipulation, and external negative ionization in 1995) have further increased the sensitivity and specificity of ion trap MS. The combination of these developments has pushed the detection limits for modern mass spectrometers well into the part-per-trillion range and, in some cases, even part-per-quadrillion range. Direct Sampling Ion Trap Mass Spectrometry (DSITMS) further combines high speed sampling with the high speed and sensitivity of the ion trap. DSITMS instruments (developed at ORNL) are field transportable ion trap mass spectrometers and can be used to rapidly detect a wide range of compounds in addition to explosives. In almost all cases, detection limits in the low pg range (or sub ppb range) has been achieved for these compounds. An additional, attractive feature of ion traps is that by alternatively using the more selective MS/MS technique (and thus dramatically lowering the rate of false positives), comparable sensitivities are still obtained due to the ion storage nature of ion traps. The direct sampling interfaces which have been developed allow the analysis to take place in real time or a few minutes as compared to tens of minutes required for typical GC/MS analysis. 


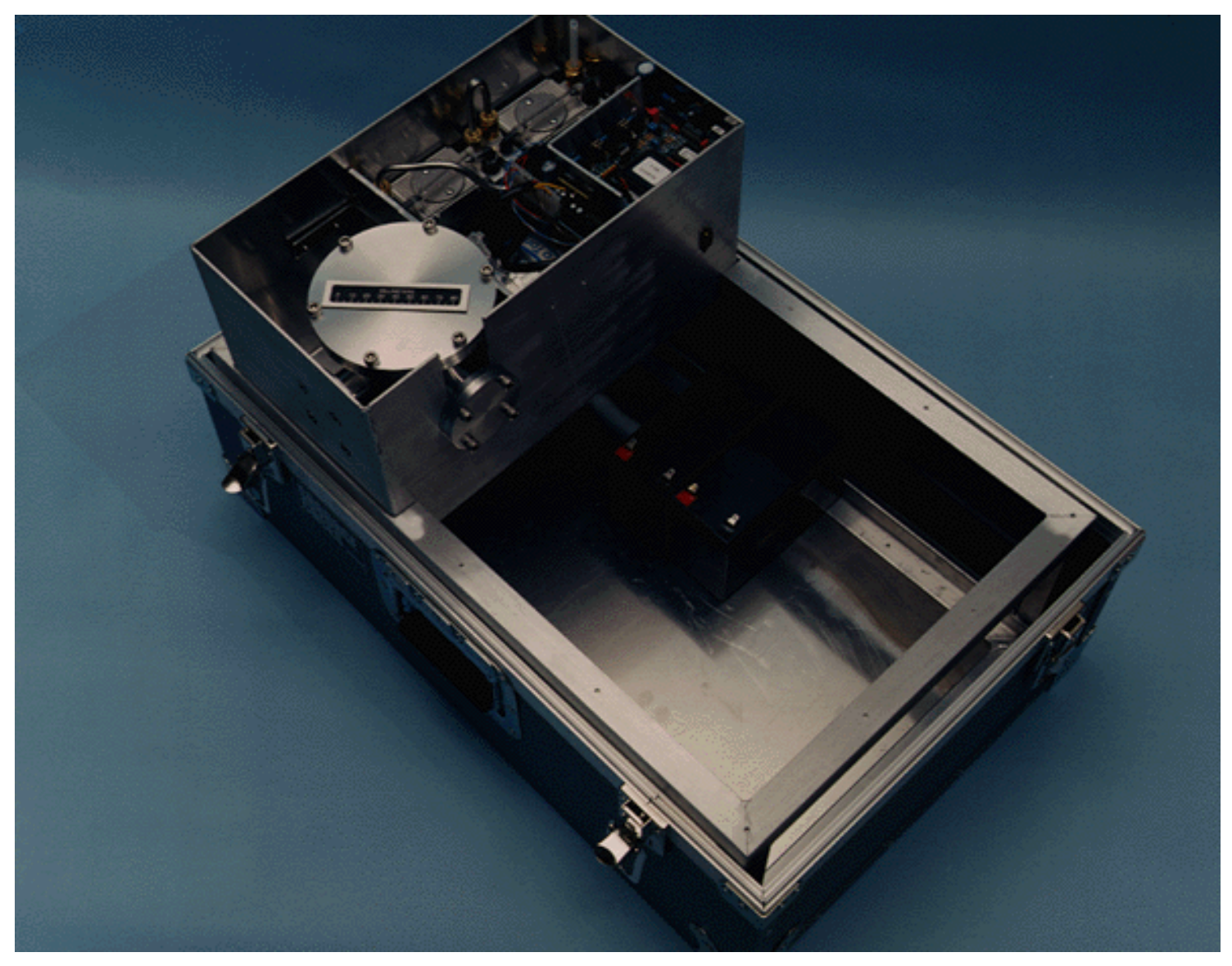

Fig. 15. Direct Sampling Ion Trap Mass Spectrometry.

\section{Technical Status/Maturity}

Current funded DSITMS research

U.S Army Environmental Center - DSITMS detection of environmental contaminants and explosives detection from soil and water $\$ 200 \mathrm{~K}$

U.S Army Corp of Eng. - DSITMS detection of environmental contaminants in ground water using cone penetrometer $\$ 100 \mathrm{~K}$

U.S Army - Detection of CWC related compounds using PITMS (Treaty version of Army's CBMS)- \$250K

DOE - Development of man-portable (including battery power) ion trap mass spectrometer - $\$ 400 \mathrm{~K}$

\section{Equipment Availability}

Direct sampling ion trap instruments (except man-portable ion trap) are immediately available for laboratory or field demonstration. 


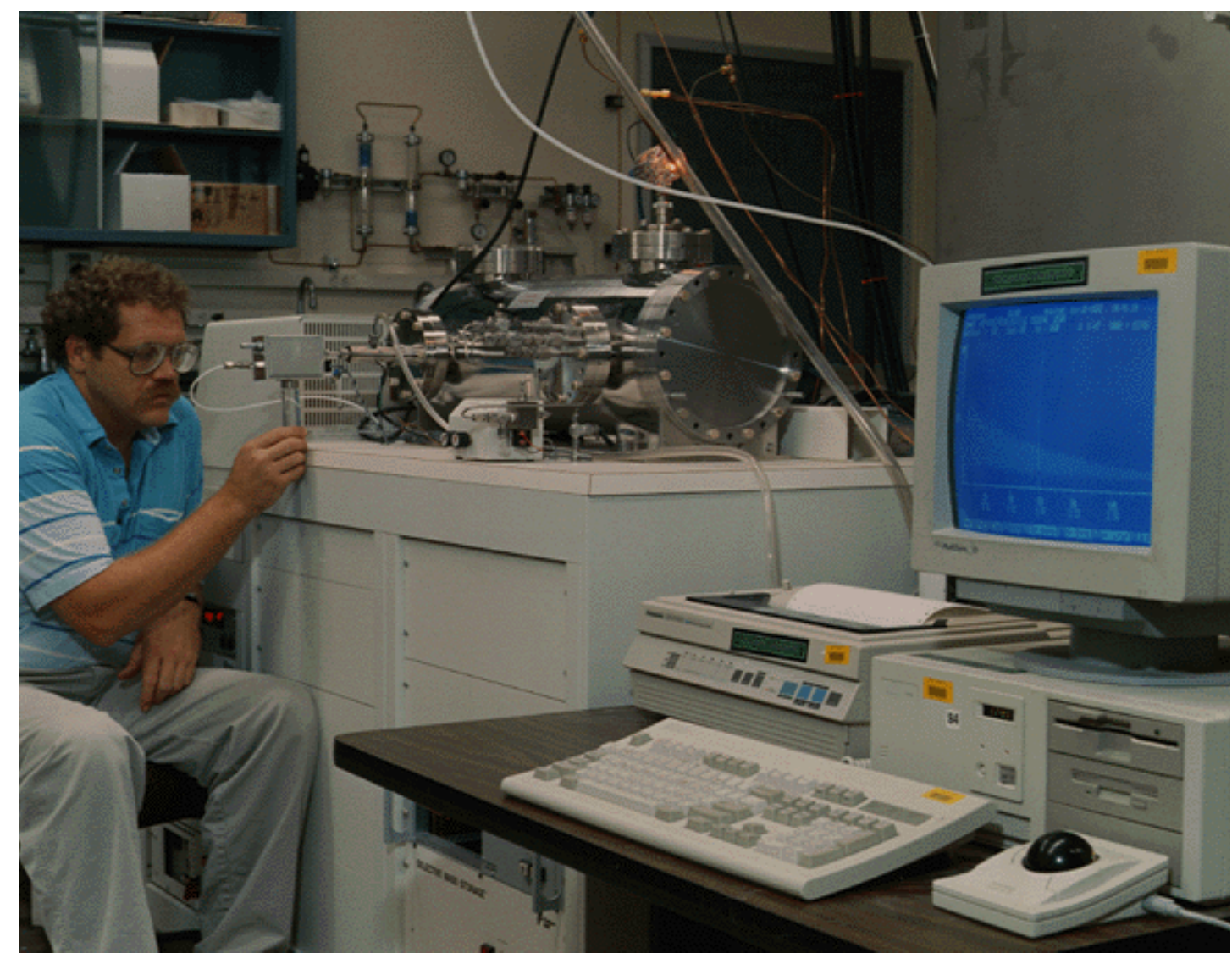

Fig. 16. Ion Mass Spectrometry System

\section{Applications and Testing}

Field portable DSITMS have been successfully demonstrated at numerous DOE and DOD sites including: ORNL (all sites), Savannah River Plant, Portsmouth, Hanford, Rocky Mountain Arsenal, Arnold AFB, Dover AFB, Wurtsmith AFB, Aberdeen Proving Grounds. In addition, the US Environmental Protection Agency has included DSITMS under Conditional Method \#8265. This method has been approved by Organic Methods working group for analysis of VOA's in water. The method will be expanded this summer to include soil, air, real-time monitoring.

\section{Note}

Novel ion trap analyzer cell geometry has been discovered which may increase the sensitivity of the ion trap by 1-3 orders of magnitude. We are currently seeking funding for this project and as such, this project remains at a conceptual stage.

\subsection{ANTIPERSONNEL LANDMINE DETECTION USING NANO EXPLOSIONS ON MICROCANTILEVERS}

\section{Technical Contact}

Thomas Thundat 
Health Science Research Division

Oak Ridge, TN 37831-6123

Phone: (865) 574-6201

E-mail: UGT@ORNL.GOV

\section{Description of Technology}

Microcantilever sensor technology is an upcoming sensing technique with broad implications in chemical, physical, and biological detection. The mass of the microcantilever is a few nanograms. The typical dimensions of these microcantilevers are 50 -200 $\mu \mathrm{m}$ long, 10-40 $\mu \mathrm{m}$ wide and 0.3 $-3 \mu \mathrm{m}$ thick. Many chemical sensors based on microcantilevers have been demonstrated with ppb sensitivity by utilizing mass adsorption or induced stress. Although ppb sensitivity is considered excellent for most applications, a million times better sensitivity is essential in detecting molecules of low vapor pressure materials such as explosives. Here we propose a variation in cantilever approach that can yield a parts per quadrillion sensitivity.

The proposed microcantilever involves a silicon cantilever that is located in a small chamber. Coating one side of these cantilevers with a different material, such as a metal film, makes them extremely sensitive to temperature variations. The bending of the cantilever can be detected by an optical method. As the temperature of the microcantilever changes, the cantilever deflection changes due to the bimetallic effect and can be detected with sub-nanometer resolution. A temperature variation $10^{-6} \mathrm{~K}$ can be easily detected by the microcantilever method. The estimated minimum detectable power level using an unoptimized cantilever was of the order of a pW, corresponding to a detectable energy of femto Joule (fJ). However, using an optimally designed cantilever, the sensitivity may be improved even further.

In this technique air from the vicinity of a suspected explosive is drawn into a chamber containing the cantilever. As the sampling continues the molecules of explosive vapors adsorb and accumulate on the cantilever surface. At the end of sampling the cantilever is heated gradually to a high temperature by a piezoresistive track implanted in the cantilever. Since the thermal mass of the cantilever is extremely small, a cantilever can be heated to a few hundred degrees centigrade and cooled to an ambient temperature in a few seconds. As the cantilever is heated, the adsorbed molecules of explosives undergo combustion producing a large and sudden variation in the cantilever deflection. A plot of normalized cantilever bending, with respect to a reference cantilever, will show a peak if explosive molecules are present.

Since the cantilevers are extremely small, a continuously operating device could be built to avoid any dead time by having an array of cantilevers sampling at different rates. The cantilever sensitivity can be improved by optimization techniques and by using catalysts for increased adsorption on the cantilever surface.

\section{Technical Status/Maturity}

Proof-of-principle experiments are presently underway for TNT and RDX . Preliminary results are very encouraging.

This program is supported by Internal Laboratory Funding.

We are expecting laboratory demonstration in about two months. 


\section{Landmine Detection by Nanoexplosion Microsensors}

\section{-Silicon technology}
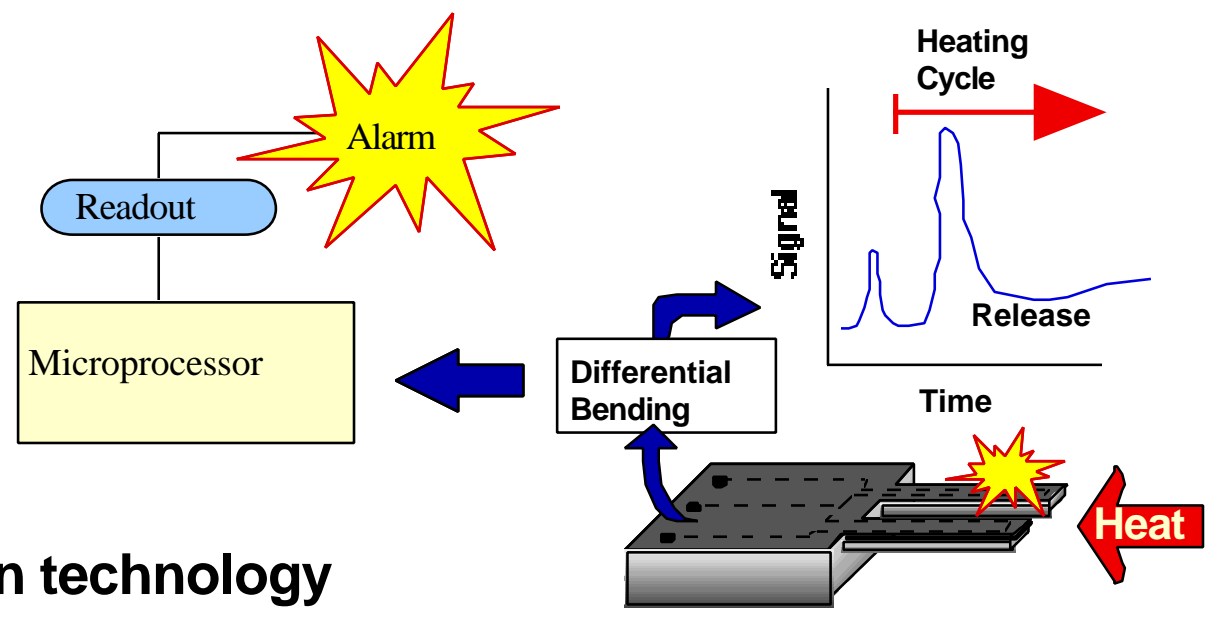

-Microchip manufacturing

-Monolithic arrays capable

-Inexpensive, low power

-Small volume

-Rugged

-High sensitivity

-Fast response, low mass

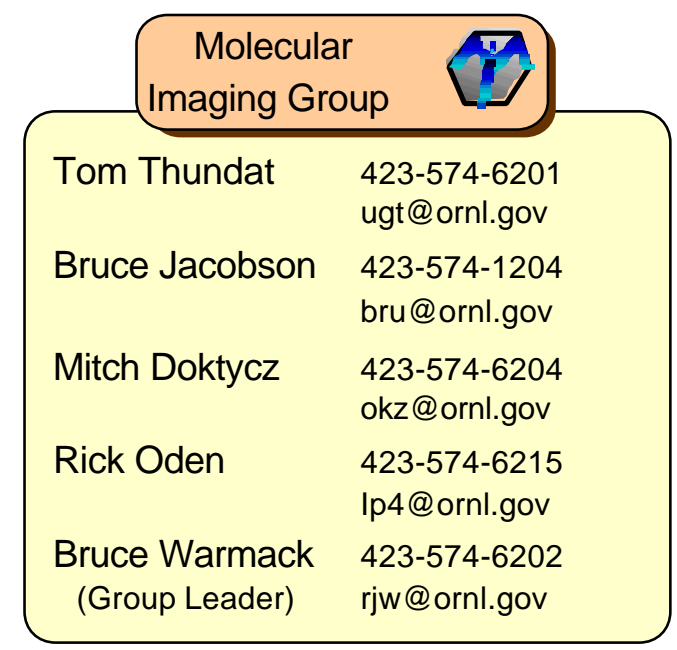

Novel sensors with extreme sensitivity for vapors from hidden explosives can be readily constructed using micromachined cantilevers. The physical principle of detection is based upon the heat released during induced nanoexplosions of adsorbed vapors on specially coated cantilevers. By using bimetallic cantilevers, temperature changes of less than 10 $-6 \mathrm{~K}$ can indicate reaction energies of $10-15 \mathrm{~J}$. Sensitivity of parts-per-quadrillion to TNT and RDX can be approached.
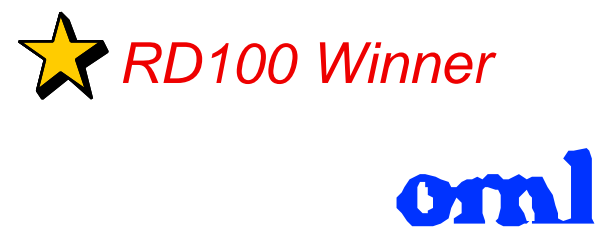

Fig. 17. Landmine Detection by Nanoexplosion Microsensors 


\subsection{MINE DETECTION SYSTEM: MOBILITY PLATFORM AND SYSTEMS INTEGRATION}

\section{Technical Contact}

James H. Hannah

Robotics \& Process Systems Division

Oak Ridge National Laboratory

Phone: (865) 574-7148

E-mail: hannahjh@ornl.gov

\section{Description of Technology}

An effective mine detection system (MDS) will incorporate a number of subsystem technologies that perform sensing, mobility, communications, and human-machine interfacing functions in an integrated system operational capability. The mobility platform and operator interface subsystems are the principal physical and functional integration points for the realization of a general-purpose MDS that can be configured for multiple mission requirements. This project will address the mobility platform and the overall systems integration of the total MDS effort.

\section{Mobility Platform}

A multi-functional mobility platform for the deployment of multiple mine detection sensor technologies will be designed and developed. A study will be performed to evaluate existing vehicle platforms and their application to the mine detection mission. Emphasis will be placed on the use of existing inventory items. The platform will be designed to support on-board sensor processing, control, and data communications functions. Careful attention will be given to practical considerations of field transport and deployment. The mobility platform will address all systems requirements as they are developed early in the project. Preliminary considerations include the following:

\section{(a) Sensor Deployment}

The state of the art in sensor technologies coupled with the wide range of possible deployment geography's means that the MDS will most likely involve the use of different types of sensors for specific missions. The mobility platform and its sensor interfacing electronics should be designed to accommodate a range of sensor types. The system design should be mechanically and electronically "modularized" to facilitate quick mission reconfiguration. Multi-sensor integration will be employed in the processing of all the sensor data to achieve maximum reliability in mine detection and location. This sensor fusion will be implemented in a manner to accommodate the most efficient human-machine interface, readily adaptable to changing mission requirements.

\section{(b) Automatic braking}

Automated braking functions will be needed to assure that the MDS has an acceptable response time in the event of a positive detection. When the vehicle is moving at 5 miles per hour, there is just 2.7 seconds for it to stop once the mine has been detected. Reliance on the operator for such a quick response may not be possible through remote control.

\section{(c) Vehicle operation}

There are a couple of deployment scenarios for the mine detection system. Depending on the area of the mine field, time constraints and specific operational requirements, one can envision either a manual, a teleoperated, or a fully automated vehicle. 
- Manual operation: During this operational scenario, the vehicle will be driven by a soldier. He will have complete control of the operation, except when a mine is detected during the scanning process, wherein the brakes will be automatically applied and the operator will have to override the safety controls to make the vehicle mobile again.

- Teleoperated vehicle: In case of teleoperated vehicle, the operation of the vehicle is controlled by a soldier using a carefully designed human-machine interface subsystem. The philosophy is to have a soldier-inthe-loop during the entire mine detection operation. The operator can control the vehicle from a remote location and will be given feedback of the location, the field condition, etc., through cameras and other driving sensors. He will be able to fully maneuver the vehicle to any location and also bring the vehicle back from the mine field once the operation is completed.

- Fully automated operation: Here, once the location of a mine field is determined, the vehicle can be deployed in an automated mode where the system is programmed to sweep a designated area unattended. The vehicle will be fully automated and will have decision making algorithms to change its path once a mine is detected. The vehicle navigation systems will utilize GPS execute the designated sweep trajectories and to record the locations of detected mines. Path planning tools in conjunctions with digital terrain maps will be provided to assist operators in setting the system up for automated sweeps

\section{Systems Integration}

Systems integration deals with the challenge of bringing together the parts of a system into a successful working unit, and the process of systems integration is vitally important during all phases of development, design, and implementation. The importance of systems integration is directly proportional to system complexity. The mine detection system will be complex as innovative sensor and mobility technologies are combined to increase detection rates, to incorporate automation, and to address a wide range of theater operations. Experience has shown that critically important tradeoff synergy occurs during development, design, and implementation at the subsystem and the overall system levels. For example, sensor deployment, or mobility, factors may be pivotal regarding the feasibility of specific sensors (e.g., certain standoff positioning flexibility and precision may be necessary for a specific sensor to meet detection criteria). Modern engineering experience has shown repeatedly that effective systems engineering and integration are critically important for the successful realization of complex systems.

Systems engineering and integration technologies will be used to assure that across all of the subsystems and candidate technologies that hardware/software requirements, interfaces, development activities, designs, fabrication/assembly preparations, and testing procedures are met, or accomplished, comprehensively, efficiently, and effectively. Systems engineering and integration practices will be used in rational tradeoff evaluations and overall performance optimization at the mine detection system level.

\section{Related Accomplishments}

Over the past twenty years, ORNL has developed many remote vehicles and has "fine tuned" the application of effective systems integration practices to robotics and automation systems prototype development including full-scale systems. Numerous systems have been developed for both the DOE and the DOD. In

United States Army projects, eight major systems have been developed that involve applications in battlefield robotics research, robotic Patriot missile canister handling, robotic tank ammunition loading, automation of cargo handlers, remote excavation, remote explosive ordnance disposal (EOD), and low-data-rate remote vehicle driving. In the following discussion, more detail about several of these projects is provided. 


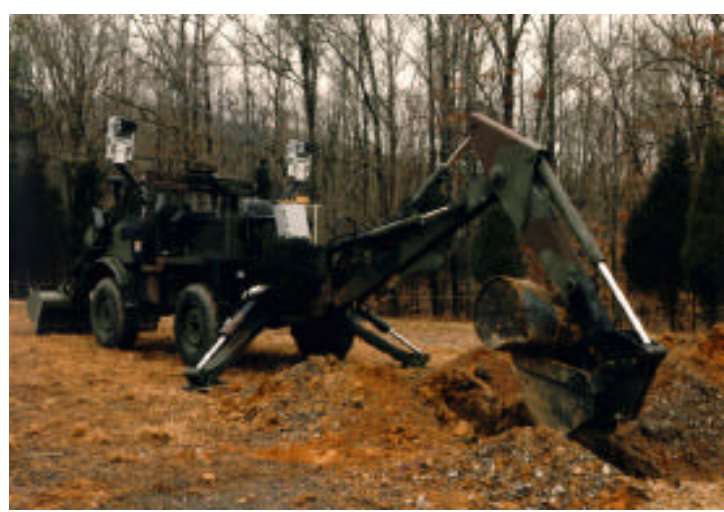

Fig. 18. Teleoperated Small Emplacement

\section{Excavator}

The Teleoperated Small Emplacement Excavator (SEE) Project was a joint effort between the Army and the DOE. This project produced a remote control kit for fielded SEE units, which will allow EOD technicians to accomplish clearing and digging operations from a safe standoff distance. The teleoperated SEE uses real-time digital controls, wireless data and video communications, and an innovative portable operator control station to allow soldiers to "dig" remotely with essentially the same proficiency.

The Future Armor Rearm System (FARS) was a technology demonstrator of an armored tank reloading system that could reload M1-class tanks in forward positions. A large manipulator system with an integral belt conveyor transports rounds from the supply vehicle to the tank. FARS utilizes an on-board digital control system based on commercial off-the-shelf technology to allow crews to deliver tankrequested mixes of heat and kinetic

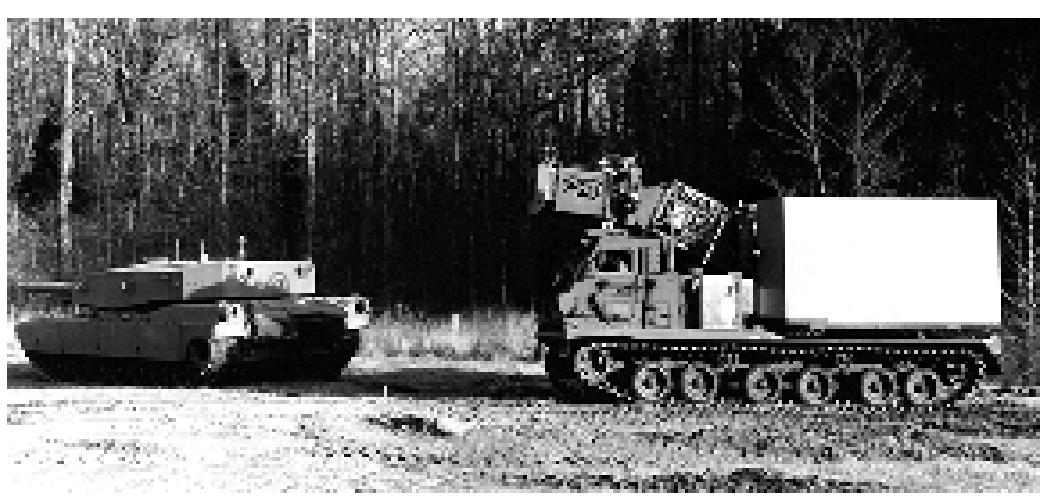

Fig. 19. Future Armor Resupply Vehicle. rounds. FARS was one of three projects that focused on the future ammunition logistics automation. The Modular Artillery Ammunition Delivery System (MAADS) addressed the fundamental subsystems necessary for a field resupply vehicle for the Crusader howitzer. The Advanced Integrated Rearm System (AIRS) was a proof-of-principle activity directed at feasibility demonstration of automated processing of artillery rounds on board the Future Armored Resupply Vehicle.

ORNL has also been involved in smaller-scale mobile systems for survey and characterization work in DOE buried waste remediation and Army EOD applications. The Soldier Robot Interface Project (SRIP) vehicle was a joint effort between ORNL, the Tooele Army Depot, and the Army Research Laboratory. SRIP is a remotely operated and tetherless vehicle, which has been used to explore battlefield robotics missions including damage assessment and evaluation. SRIP results were used to develop the joint DOE and DOD remote characterization system (RCS), which has been used as a sensor platform to characterize nuclear buried waste sites and firing ranges.

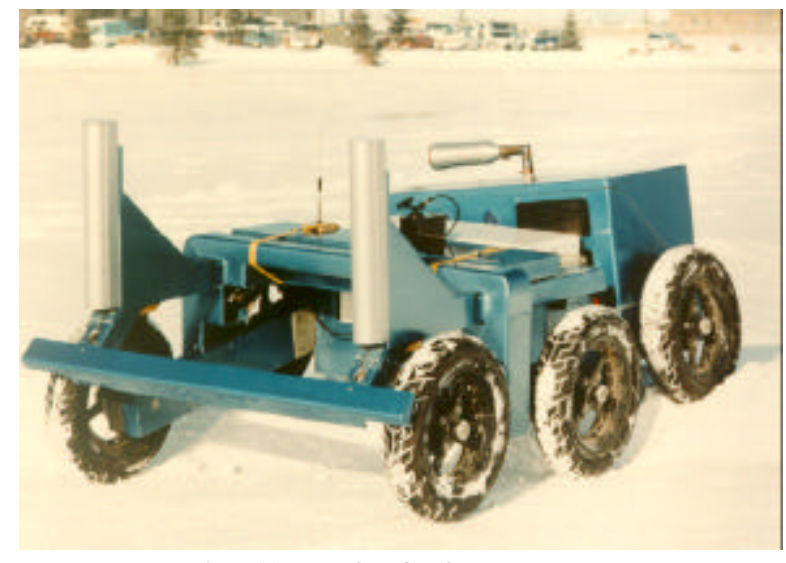

Fig. 20. DOE/DOD Remote Characterization System.

RCS is basically a mobile sensor platform and includes many special vehicle features intended to enhance sensor performance. RCS was designed with minimal nonferromagnetic to enhance the performance of 
magnetic underground detectors. ORNL's responsibility was the overall RCS control system and humanmachine interface.

These are examples of large projects where ORNL staff have worked directly with Army personnel in all project phases from the development of requirements through prototype evaluation. Effective systems integration was essential to achieve project objectives within budget and schedule. Perhaps more importantly, effective systems integration was essential to ensure that the system's performance was optimized with respect to technology options while adhering to system requirements.

\section{Facilities and Resources}

The Robotics \& Process Systems Division at ORNL has the laboratory infrastructure and high-bay facilities necessary to accomplish effective systems integration. The high-bay facilities provide $7800 \mathrm{ft}^{2}$ of floor space, which has $40 \mathrm{ft}$ of head space and access by two 10-ton overhead cranes.

In the proximity of these areas are smaller development laboratories with services and provisions for smaller scale development and testing. Throughout the complex, there are 12 Sun engineering workstations and 6 Silicon Graphics workstations that are routinely used for real-time software development, design, simulation, and analysis. Shop facilities for all manner of mechanical and electrical and electronics fabrication are also available.

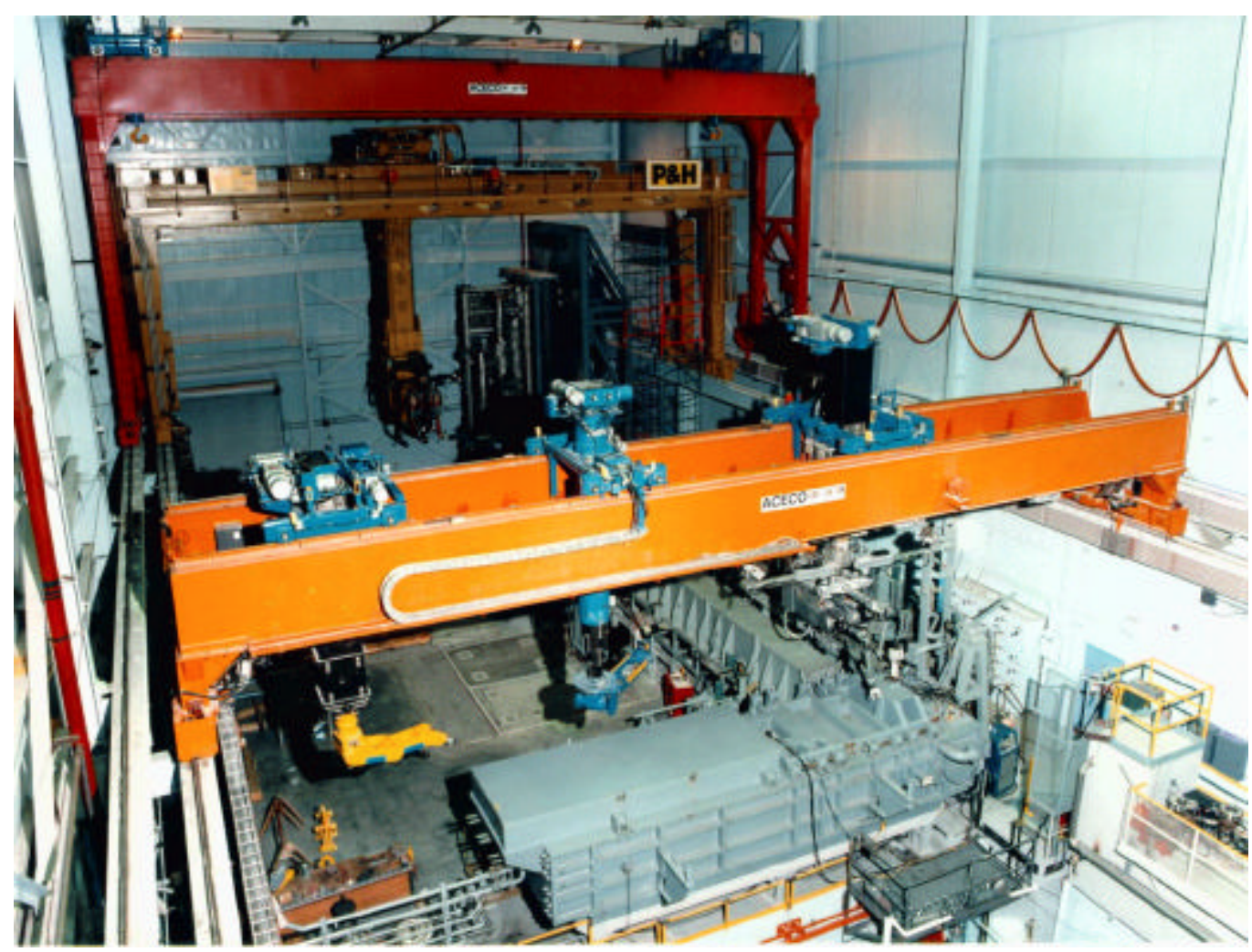

Fig. 21. Robotics \& Process Systems Division's highbay facility. 


\section{Technical Status/Maturity}

ORNL has over 200 person-years of direct experience in the systems integration of prototype systems of the magnitude and nature of an advanced mine detection system. The principles and practices are fully mature and available within the existing staff and their working resources. Standardized software/hardware design management and rapid prototyping tools and practices are available for the efficient exploration of technology tradeoffs. ORNL, in general, has a long and successful track record in combining research with engineering in complex problems. Specific expertise pertaining to mine sensing and detection will have to be established.

ORNL has worked with a number of military and commercial vehicle platforms including the development of first prototype chassis. Platform technology is mature and ready for the MDS application. The tradeoffs pertaining to mission objectives and deployability will impose engineering challenges in the areas of tetherless operation, on-board power supply, weight, and volume.

\subsection{ADVANCED SIGNAL PROCESSING AND DATA FUSION FOR MINE DETECTION}

\section{The Present State Of Progress In Automatic Mine Detection}

Despite work performed by many organizations, there has been no significant breakthrough in electronic mine detection since the Korean war. The combination of magnetometers and physical ground probing is presently the most effective method for detecting mines. Sensing methods other than metal detectors have been tried but have demonstrated limited success, mostly due to the relative weakness and inconsistency of the characterizing emanations and the wide variation of soil and environmental conditions in which mines are encountered.

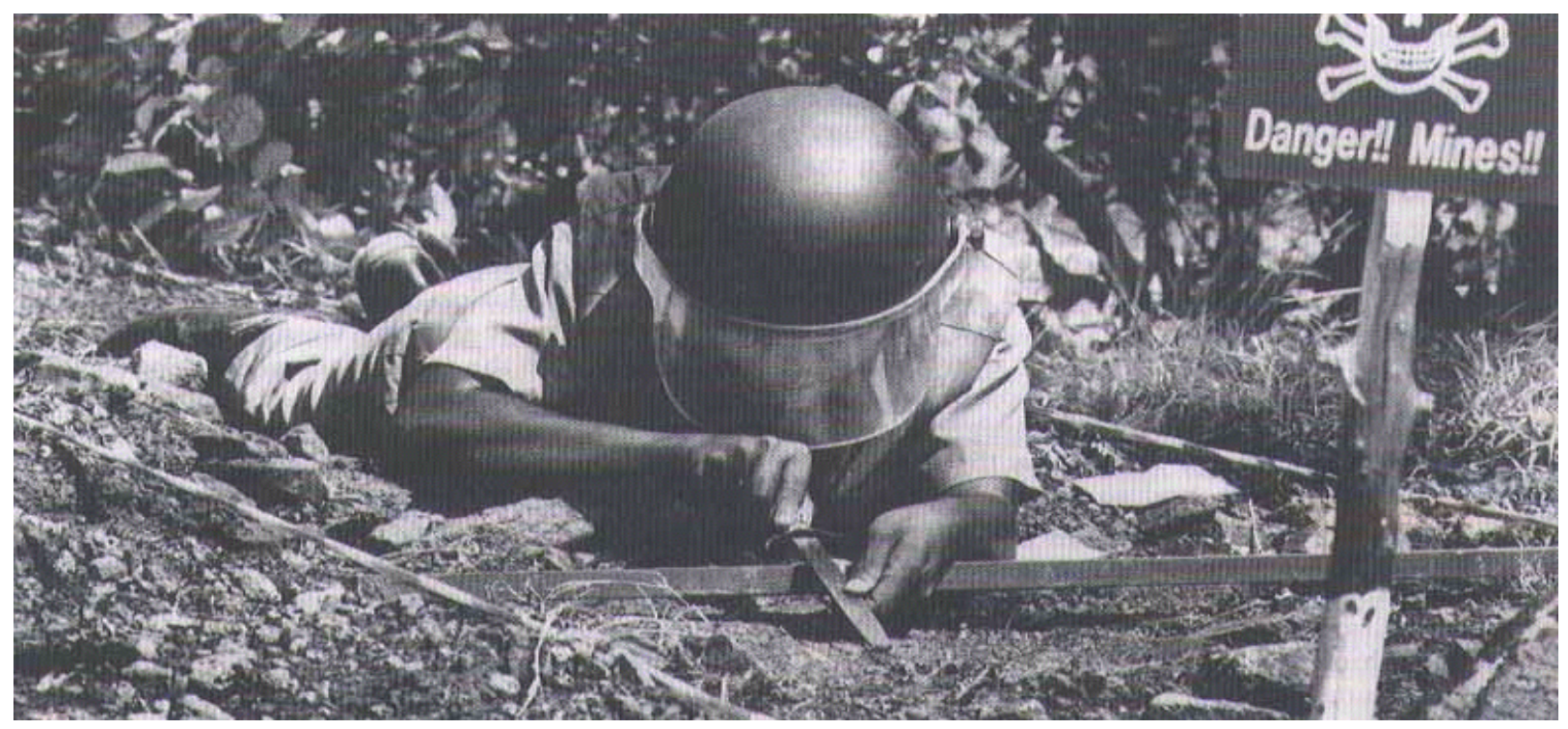

Fig. 22. Hand probing for land mines. 


\section{The Fundamental Unsolved Problem}

One reason for this lack of substantive progress is clear. The most critical necessity for any electronic identification system is a feature space in which identifying features are well characterized. Discovering a suitable feature space is the most difficult problem in the development of an identification system. Once a feature space is identified, everything else becomes a straightforward engineering problem. Without a feature set, automatic identification or classification systems are certain to fail. No specific feature set for mine signatures has ever been identified.

Despite the fact that past research has failed to turn up a consistent feature set, there is reason to believe that such a feature set exists. The dog is the most consistent mine detector. It is not known what features the dog identifies, but it is suspected that the dog uses a wide variety of sensory cues. Hence, identifiable features exist, even if we have not identified them.

Beyond the argument that if dogs can do it, it must be doable, the following are some specific reasons to suspect that an identifiable feature space exists. The Pemex robotic ultrasonic system occasionally blows up robots, but it does suggest that there are some useful features returned by ultrasonic systems. Broadband pulsed radar produces some useful features; the weakness in the broadband pulsed radar tomographic system recently reported in the literature is in the signal processing, not the physics of the sensor. Geophysical effects tend to be offsetting; effects that disrupt electromagnetic waves are not excessively disruptive to ultrasonic waves, and vice versa. Thus, a system that uses both effects simultaneously should provide a more consistent indication than a system using either effect alone. The use of both effects in concert with magnetometer data should be much more reliable than the magnetometer alone.

Related to the feature identification problem, is the effect of the geophysical environment on the signatures. It is known that moving a sensor six inches completely changes the feature pattern. If the geophysical properties of the soil were known, either of two technical approaches might follow. First, those features that are the least sensitive to geophysical effects could be identified. Second, means to compensate for variations in the environment could be devised. In any case the effect of dirt on the identification scheme needs to be investigated.

If nothing else, an ultrasonic/electromagnetic scheme would detect disturbed earth. Digging and loosely backfilling cause a small, but consistent, change in the electrical properties of dirt. For example, the dielectric constant in a backfilled hole will always be lower than that of the undisturbed surroundings. This change should be easy to detect in a radar ground reflection.

It does not appear that a systematic study of the emanations from mines in response to specific stimuli in specific geophysical environments has ever been done. The Army evidently has no data that it can furnish to independent researchers in which to search for features. The major piece of fundamental research that needs to be done is to systematically study mines in a variety of soils for their responses, and to analyze the responses in a variety of mathematical spaces to identify their distinguishing features. ORNL has successfully done this kind of thing before; in the remote airport monitor project, acoustic airplane signatures were projected onto a wavelet scale space, and airplane types were readily identifiable. 


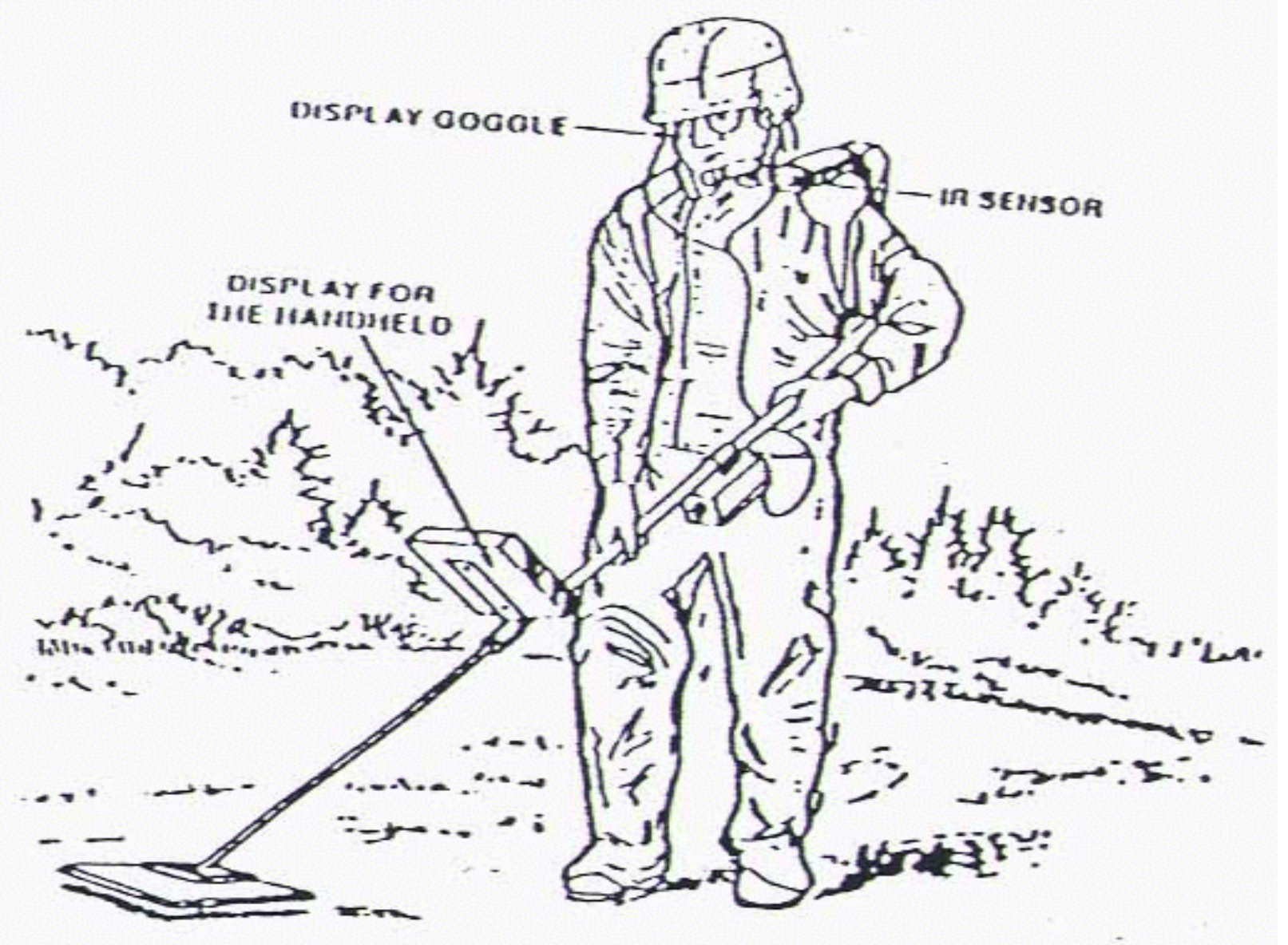

Fig. 23. Future Landmine Detection System.

Despite these unanswered fundamental questions, a variety of high-tech development activities are under way. Imaging is the primary signal processing method used in almost every high-tech development reported in the recent literature, including the man-portable scheme presently under development for the Army. These systems do not recognize the signature of a mine automatically. They depend on operator judgment. The penalty for misjudgment is instant death or catastrophic injury to the operator.

These high-tech systems evade the fundamental unsolved problem of unidentified features by having the operator do the feature extraction and classification in real time in his brain from a machine-generated image. This is in principle no different from the dog. The primary difference is that the human requires high-tech enhancement of his senses, and the dog does not. Also, the human is aware of the risks while the dog is (apparently) not. This suggests that the dog is in a better position to make a dispassionate judgment.

The feature identification and geophysical problems are fundamental. Until they are solved, high-tech developments in mine detection will usually fail. Any successes will inevitably represent minor incremental improvements over present methods. 


\section{Wavelet Analysis}

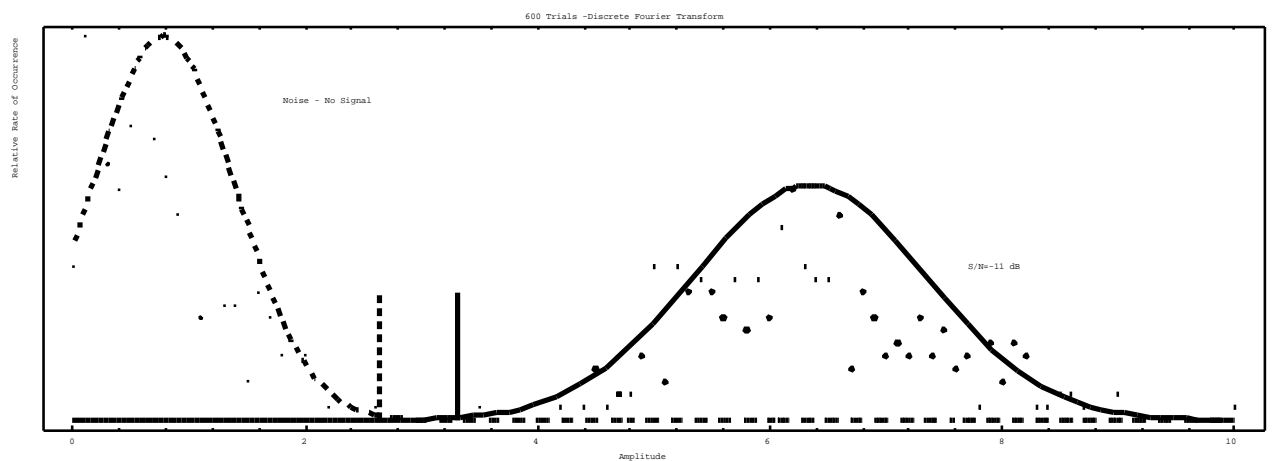

Fig. 24. With one cycle of discrete Fourier processing, a -11 DB S/N signal can be readily identified.

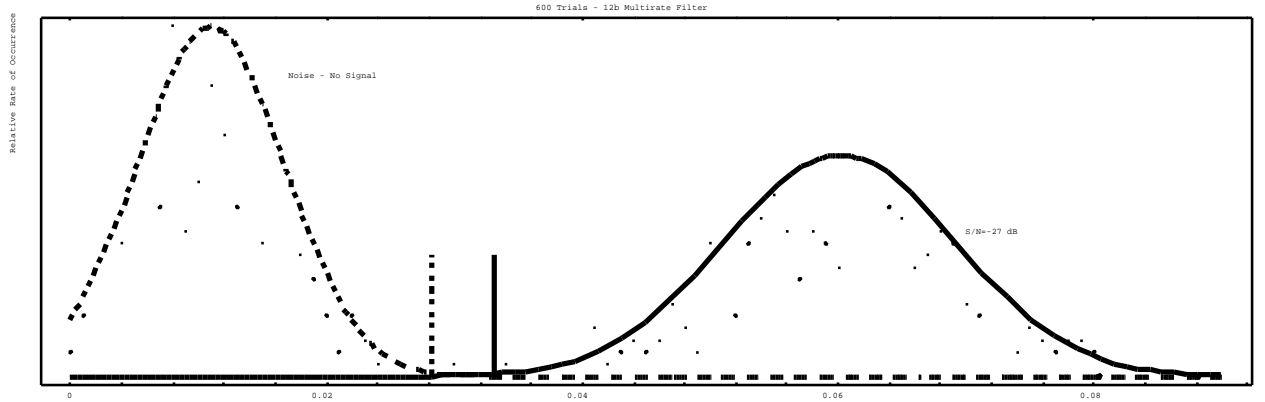

Fig. 25 With one cycle of wavelet/curve fit processing, a -27 DB S/N signal can be readily identified.

Virtually all present practice in signal processing for mine detection is based on taking a data stream and increasing its volume without adding information. Typically these systems construct an image, or hologram, or tomogram. A computationally cheaper method would be to use a signal processing algorithm that removes data at each step in the process while preserving the information. In particular, it is desirable to keep the data stream one-dimensional (1-D).

Signal processing is most effective when data are projected onto orthogonal subspaces. The idea is that it is necessary to decorrelate the desired features from the undesired features, and then ignore the undesired features. This is at the heart of orthonormal wavelet analysis.

Wavelet analysis is good, fast and cheap, all at once. It is based on constant-Q filtering. Since many physical processes are constant-Q in nature, wavelets are powerful for sensing applications. Also, orthonormal wavelet analysis is implemented with cheap digital filters, and is much faster than Fourier analysis.

A particular virtue of wavelet analysis is its sparse use of data. Each step in the process discards data while preserving information. The arcane logical constructions underlying wavelet theory give assurance that all unnecessary data are discarded as soon as possible, and that none of the underlying information is lost. Discrete wavelet analysis inherently minimizes the number of needed signal processing steps. 
Wavelet techniques are useful in both in the areas of performance enhancement, and in the extraction of previously unextractable information. Thus, it is possible that wavelet analysis could be used to enhance the present generation of mine detection systems through data compression, denoising, etc. Also, as has been the case in other areas, it is imaginable that projecting mine sensor data onto a suitable wavelet scale space could lead to the discovery of a consistent feature set where none has been found before.

\section{ORNL Advanced Signal Processing Capabilities}

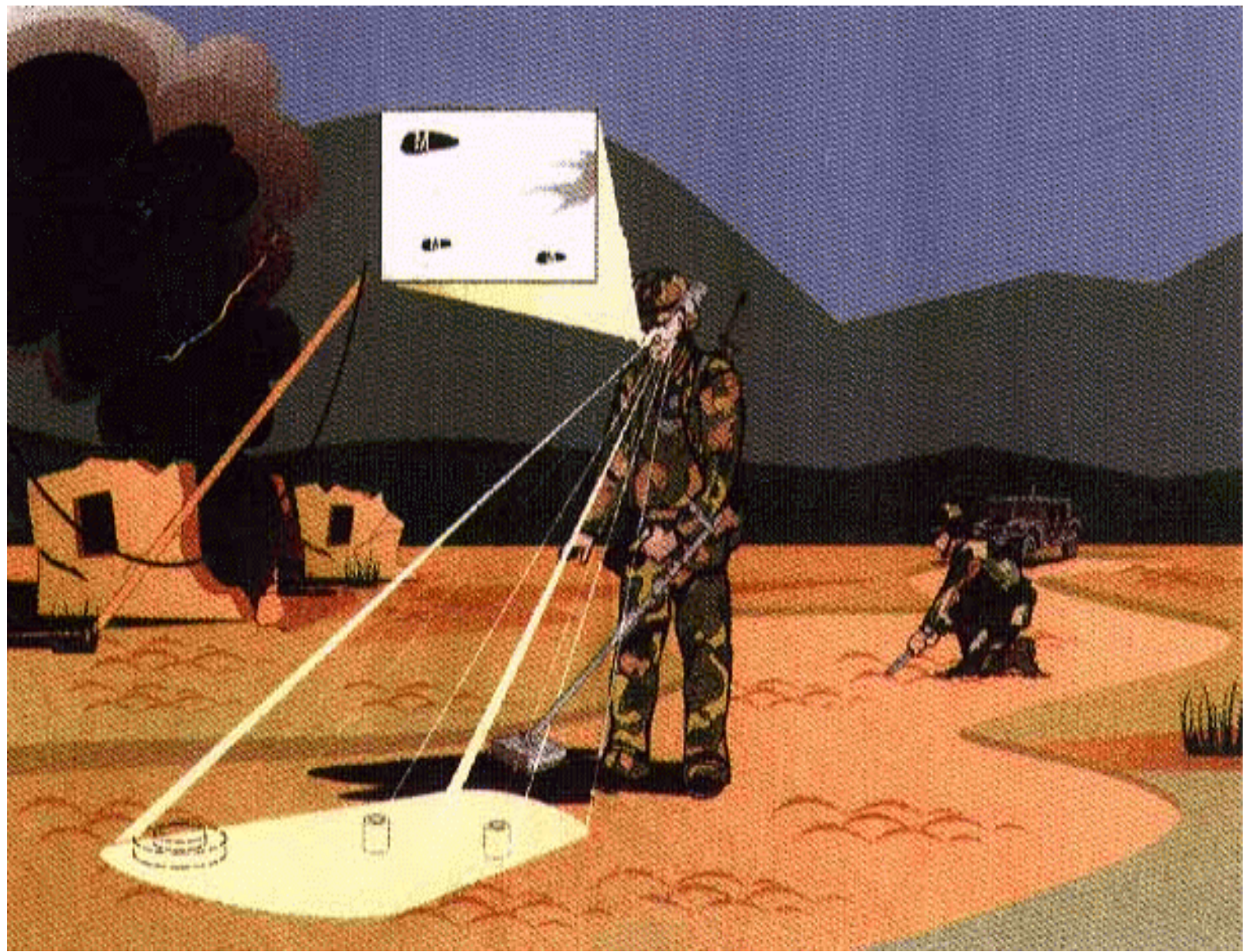

Fig. 26. Future Landmine Detection System.

The technologies of advanced signal processing and sensor fusion show great promise for enhancing the ability to electronically detect mines. Incremental improvements in the performance of individual sensors have been reported by applying advanced signal processing techniques to the sensor signals. It is our belief that advanced signal analysis and signal processing techniques combined with advanced sensor fusion concepts offer a much greater potential for success in reliably detecting mines than has been realized to date. The performance of existing sensors and systems for mine detection may be enhanced greatly by applying these techniques.

The research staff at ORNL offers several unique capabilities in the areas of advanced signal processing and sensor fusion which can be brought to bear on the mine detection problem. ORNL has been highly 
successful in employing advanced signal analysis and signal processing to isolate information hidden within electronic signals.

ORNL is a recognized world leader in the application and implementation of wavelet analysis techniques, as well as other advanced analysis techniques for locating information "hidden" within sensor data for diagnostic purposes. One example is the use of wavelet analysis in the detection of a human heartbeat in an enclosed area. Each time the heart beats, it generates a small but measurable shock wave which propagates throughout the body. This wave is, in turn, coupled to any surface of the object with which the body is in contact. A velocity geophone and/or microwave detector can be used to detect the deflections imparted to an enclosed space (such as a vehicle). At the heart of the detection scheme is wavelet analysis, which is used to greatly enhance the detection of the heartbeat signal that would otherwise be buried in an extremely unfavorable signal-to-noise ratio.

Most wavelet applications appearing in the general wavelet literature, such as finding cancer signatures in mammograms, have been multidimensional. The only mention of wavelets in the mine literature was by JPL on wavelet-based image enhancement. Nevertheless, problems like feature extraction and the cleanup of sensor data are often 1-D. ORNL is a recognized leader in practical wavelet applications, and is probably the world leader in hardware-based 1-D wavelet applications. Outside recognitions of ORNL's leadership in this area include the R\&D 100 Award and several invited papers. ORNL has solved several otherwise intractable problems with wavelet analysis, including the airplane signature problem, and characterization of extremely broadband magnetic effects.

ORNL also has recognized capability in the area of sensor fusion. Sensor fusion can be used to create "virtual sensors" by combining the output of multiple sensors or sensors of different types. Research conducted at ORNL into the effectiveness of sensor fusion algorithms has produced a method for evaluating the accuracy of a sensor fusion algorithm. The method allows a bounding of the error of the fused information, and can be used to assure that the fused information is at least as accurate as the best information from any single sensor.

In addition to addressing the fundamental research questions, we believe that a combination of ORNL's advanced signal processing and sensor fusion capabilities can be used to maximize the effectiveness of existing mine detectors.

\subsection{CALIFORNIUM-252 BASED NEUTRON INTERROGATION SYSTEM FOR THE DETECTION OF ANTIPERSONNEL LANDMINES}

\section{Technical Contacts}

L. Robinson and E. Johnson

Chemical and Analytical Sciences Division

C. W. Alexander and R. C. Martin

Chemical Technology Division

\section{Description of Technology}

There is an increasing demand to perform real-time field measurements of trace and major elements in a wide range of materials. Neutron activation analysis (NAA) conducted with isotopic neutron sources such as ${ }^{252} \mathrm{Cf}$ 
for some time has played a significant role in this arena. Applications have included toxic element analysis in environmental matrices, identification of elemental components of chemical warfare agents, the detection of fissile materials by the delayed neutron technique, and explosives detection in luggage. Presently, the detection of antipersonnel landmines is of great concern for the U.S. military. Antipersonnel landmines are relatively small devices generally constructed mostly from inert plastic materials. These features render them difficult, if not impossible to detect by conventional means under many circumstances. The development of a field useable ${ }^{252} \mathrm{Cf}$ based neutron interrogation device that could be employed to detect the presence of antipersonnel landmines and add to the military's repertoire of landmine detection capabilities is possible.

Neutrons generated by a ${ }^{252} \mathrm{Cf}$ sources interact with nuclei of elements to often produce an unstable compound nucleus which quickly decays by the emission of prompt gamma-rays. These prompt gammarays are typically detected using high purity germanium detectors, or high efficiency scintillation detectors. Fortunately, certain elements such as nitrogen - a key component in many explosive materials - can be detected efficiently in certain matrices. Examples of previous applications of ${ }^{252} \mathrm{Cf}$ based systems include a field portable device for the detection of chemical warfare agents, and an explosives detection system for airport luggage. In such devices, only microgram quantities of ${ }^{252} \mathrm{Cf}$ are required making it feasible to contend with radiological safety issues.

\subsection{IDENTIFICATION OF LAND MINES USING PULSED NEUTRONS}

\section{Technical Contact}

Dr. Phillip C. Womble and Frederick J. Schultz, ORNL

Dr. George Vourvopoulos and Dr. Linus Dep, WKU

\section{Description of Technology}

During the last three years, Oak Ridge National Laboratory's (ORNL) Applied Radiation Measurements Department (ARMD) in conjunction with the Applied Physics Institute (API) of Western Kentucky University, has been developing a method for non-destructive, non-intrusive, elemental characterization. This method is being used in projects such as on-line coal bulk analysis. The method has also been demonstrated for the detection of hidden explosives and drugs (see attached publications). The researchers at API have recently been approached by the Aberdeen Proving Grounds to assist them with the identification of unexploded ordnance (UXO) at the Jefferson, Yuma, and Dugway Proving Grounds. Oak Ridge has looked into the possibility of developing a system that can identify anti-personnel mines (e.g. a PNM1 mine). Initial results (as described below) have been encouraging and we believe that this technology can detect and identify small land mines (minimum metal).

\section{Methodology}

Explosives contain hydrogen, carbon, nitrogen, and oxygen in a specific ratio to each other. Soil, depending on its composition, will contain some of these elements along with other elements, such as silicon. Similarly, other materials that can be on the surface or near the surface of the ground (tree trunks, metal pieces, pieces of plastic, newspaper bundles etc.) contain a number of elements that can differentiate them from each other. To identify these elements, neutrons and gamma-rays with large penetrating ability can be utilized. In using neutrons as the interrogating particle, one has to take into consideration that the various elements are 
preferentially excited by different energy neutrons. Based on the above, we have developed the Pulsed FastThermal Neutron Analysis (PFTNA) technique which works as described below.

A neutron generator produces a train of $14 \mathrm{MeV}$ neutron pulses, a few $\mu$ s wide. The fast neutrons impinge on the object to be interrogated, and initiate a series of nuclear reactions such as (n,n" ") and (n,p') with some elements such as $\mathrm{C}$, and $\mathrm{O}$ that have a large cross section for these reactions. The '-rays emitted from these reactions (e.g. $4.43 \mathrm{MeV}$ for $\mathrm{C}, 6.13 \mathrm{MeV}$ for $\mathrm{O}$ ) act as fingerprints for these elements. The "-rays are detected by a suitable set of detectors, and are stored in a data acquisition system. After the neutron pulse is stopped, some of the fast neutrons that are still within the object lose their energy through collisions with, primarily, the light elements contained in it. When the neutrons have an energy of less than $1 \mathrm{eV}$, they can be captured by elements such as $\mathrm{H}, \mathrm{N}$, and Fe through $(\mathrm{n}$, ) reactions. As in the case for $\mathrm{C}$ and $\mathrm{O}$, the emitted '-rays act as fingerprints of $\mathrm{H}, \mathrm{N}, \mathrm{Fe}$, etc. They are detected by the same set of detectors and are stored in a different data acquisition system. This procedure is repeated with a frequency of approximately $10 \mathrm{kHz}$. After a predetermined number of these pulses, there is a longer pause between pulses that allows the detection of '-rays emitted from elements such as Si that have become radioactive with a half-life of ms or $\mathrm{s}$ (activation analysis). Therefore, by utilizing fast neutron reactions, neutron capture reactions and activation analysis, a large number of elements contained in a object can be identified in a continuous mode without sampling.

\section{Proof of Principle}

The above method has been shown that it can be used effectively in a number of scenarios such as:

- verification of the absence of high explosives in nuclear weapon trainers;

- identification of illicit drugs hidden in sacks of coffee, sugar, or rice;

- on-line coal analysis;

- identification of UXO's; and

- differentiation between chemical weapons and conventional explosives.

To investigate whether the method can differentiate between mines and other objects that can be found on the ground or near the surface, a series of measurements was initiated. The following objects were used:

- PNMI Russian-made shell filled with 200g of ammonium carbonate used as a TNT simulant;

- $1.5 \mathrm{~kg}$ piece of plate iron (simulating a piece of a farm implement in the field;

- $20 \mathrm{~cm} \times 13 \mathrm{~cm} \times 2.5 \mathrm{~cm}$ piece of wood; and

- old tennis shoe. 


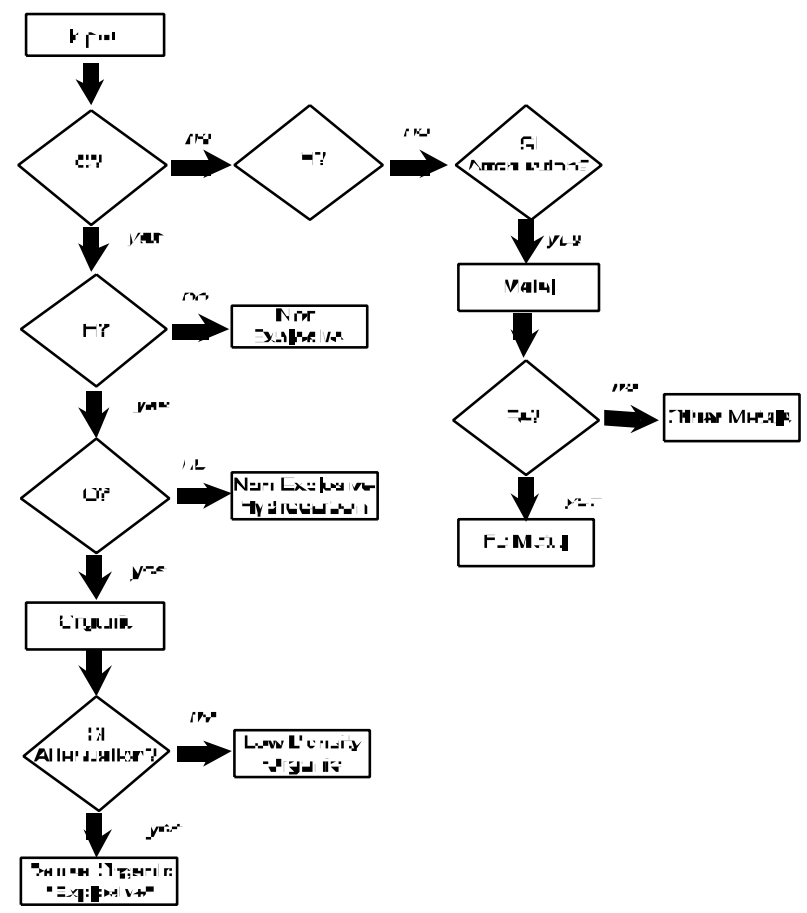

Fig 27. PFTNA decision tree schematic.

The decision-tree was based on the measurement of the chemical elements $\mathrm{H}, \mathrm{C}, \mathrm{O}, \mathrm{Si}$, and $\mathrm{Fe}$. $\mathrm{Si}$ is the only element that is not expected to be in any of the above objects. The detector acquires gamma-rays from silicon contained abundantly in the ground. If an object, however, is placed between the ground and the detector, depending on the density and atomic number of the object, there will be an attenuation of the $\mathrm{Si}$ gamma-rays reaching the detector.

The interrogated object (A PNM mine in the picture) was located in front of the BGO gamma-ray detector. The neutron generator was placed approximately $60 \mathrm{~cm}$ above the mine. The detector was shielded from the direct neutron beam with a multi-layer of borated polyethylene and lead bricks. Figure 1 shows the decision tree used in verifying the absence or presence of a mine. Figure 28 shows the experimental gamma-ray spectra obtained for the objects mentioned above (mine with TNT simulant, piece of iron, etc.). As can be seen, the experimental spectra show marked differences in the $\mathrm{H}, \mathrm{C}, \mathrm{O}$, and Si signals for the above objects. From these spectra and based on the decision tree, it was possible to differentiate the various objects without difficulty. 

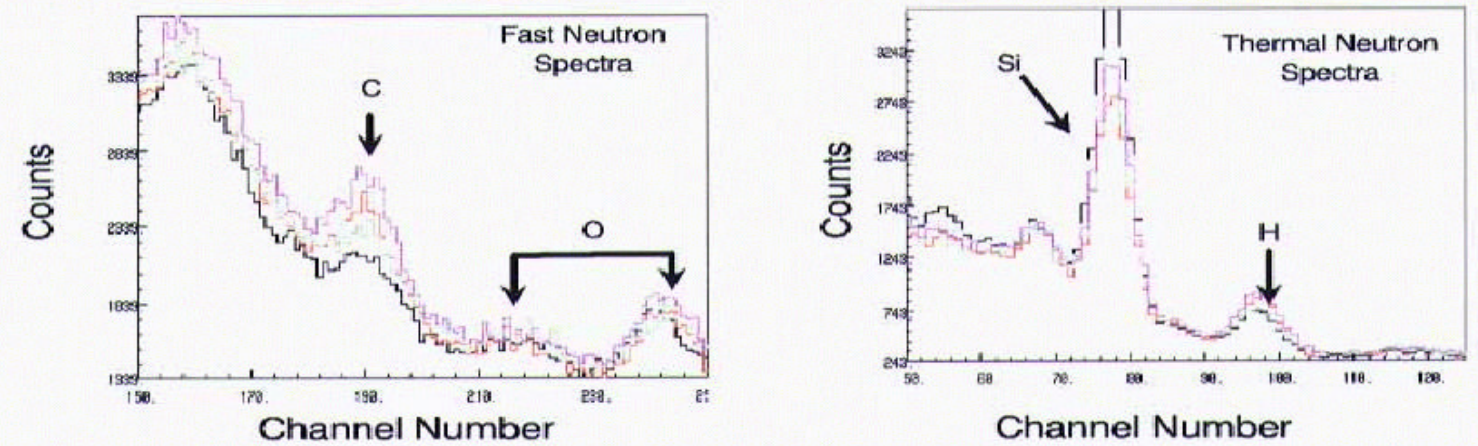

Gamma-ray spectra produced from fast and thermal neutron induced reactions. Black is background, purple is an old tennis-shoe, red is a PNM-1 mine, green is an iron plate, and blue is a piece of wood.

Fig. 28. Experimental gamma ray spectra of mine-like objects.

\section{Conclusions}

The above results indicate that, by using pulsed neutrons, it is possible to identify, with a high degree of probability, an anti-personnel mine that contains approximately $200 \mathrm{~g}$ of TNT simulant. The results were sufficiently encouraging to justify a request for funds to develop a transportable mine identification system. Given the number of the detectors that are suggested, the weight of the required shielding, radiation risk, etc., this system cannot be portable; instead, a transportable system is envisioned. Figure 29 shows a schematic view of a proposed vehicle-mounted system. 


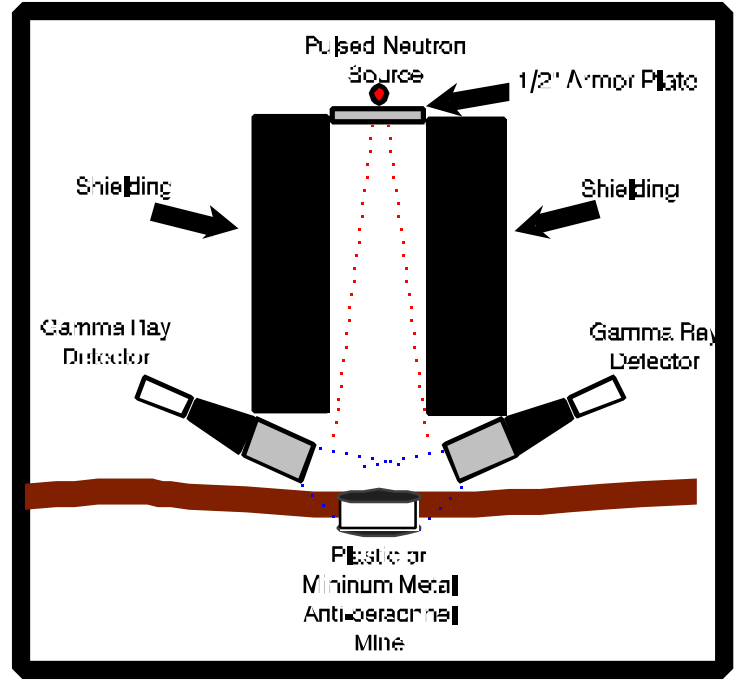

SIDE VIEW

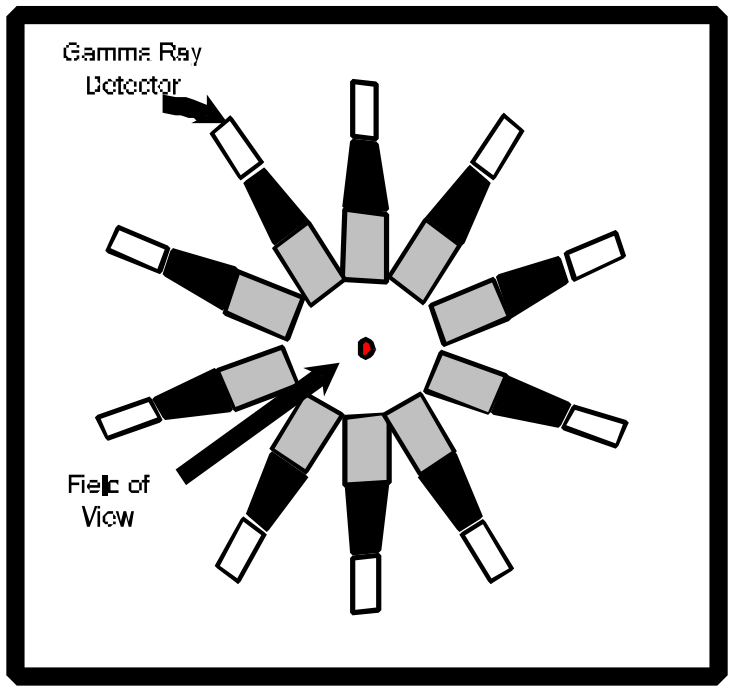

TOP VIEW

Fig. 29. Schematic view of proposed vehicle mounted PFTNA system.

The neutron generator, as well as all the data acquisition components, used for these proof-of-principle experiments, are not field-grade, ruggedized, or transportable. The algorithms used in the decision tree are not automated, requiring a certain amount of expertise for their interpretation. A fully self-contained, automated, elemental characterization system, based on the PFTNA technique has not yet been fabricated or tested. This prototype system would be transportable, ruggedized, self-calibrating, and capable of being operated by personnel with training commensurate to that provided to army personnel. The system is to provide, within 2-3 minutes, an image of the objects found within a $50-\mathrm{cm}$ radius. Two issues that need to be addressed are:

a) radiation exposure of the operators

b) risk of radiation release from the neutron generator from an accidental detonation of a mine.

\section{Funding}

Department of Energy

Other Federal Agency Sponsors

\section{Status/Maturity}

Proof-of-Principal Demonstrated

Prototype System Has Not Yet Been Built 


\subsection{COUNTER MINE SITUATIONAL AWARENESS}

\section{Technical Contact}

Steve Packard

Phone: (865) 574-9388

Fax: (865) 574-0792

E-mail: pkd@ornl.gov

\section{Description of Technology}

For the last couple years, Situational Awareness has been the key phrase for discussions of target acquisition, target hand-off, and tactical command and control. The concept of informing all concerned of the exact location of a target is equally applicable to the exact location of mines.

ORNL could develop a prototype device to cause mine locations to be reported to the situational awareness system of choice from the time and location of detection.

The mine detection situational awareness prototype (MDSAP) would be integrated into the mine detector, capture best possible locations, generate mine reporting message, and transmit mine reporting message.

\section{Integration}

The MDSAP would consist of a single printed circuit card bearing a small number of electronic components. This PC card could be developed to fit into a new mine detector as part of that development or could be retrofitted into an existing mine detector. The MDSAP would require very low power which could be provided by the host mine detector. The existing circuitry of the mine detector which stimulates an audible or visual signal would simultaneously stimulate the MDSAP to initiate its reporting process.

\section{Location}

The preferred approach to capturing geo-location is to use an existing military device, such as the one which feeds EPLARS or IVIS with global positioning data. If this is not possible, a commercially available Global Positioning System device could be acquired and integrated. Upon stimulation by the mine detector, the MDSAP would capture the location from the GPS device. This location would be no more or less accurate than other geolocation data in use by the selected situational awareness system.

\section{Message Generation}

The MDSAP would maintain a message formatted for use by EPLARS, IVIS, or another situational awareness system. Upon capture of the geo-location data, the MDSAP would convert (if necessary) to the preferred coordinate scheme and insert that data into the pre-formatted message. If the selected situational awareness system does not currently have a message format for mine location reporting, we would develop one in cooperation with the appropriate military program management staff.

\section{Message Transmission}

After inserting location into the message, the MDSAP would transmit the complete message to the selected situational awareness system using the communications protocol of that system. 
The simplest approach to this development would be to obtain a device of the lowest level of reporting for EPLARS (PLGR), IVIS, or other system and use its components to capture location and format and transmit the message. As with the possible need to develop a message format, the selected situational awareness system may not currently include display commands and symbols for mine location. We would develop these in cooperation with the appropriate military program management staff.

\section{The Benefits of MDSAP Approach}

No new separate device is introduced. Unless some increased complexity is desired, there is no additional training for the mine detector operator or users of the situational awareness system. There is no additional piece of equipment to transport or maintain.

No new communications system is introduced. This approach assumes minor increase of brief transmissions over existing systems.

Mine locations become available to the tactical unit commander as rapidly as do locations targets and friendlies and on the same screen. This makes the commander that much more aware of the situation.

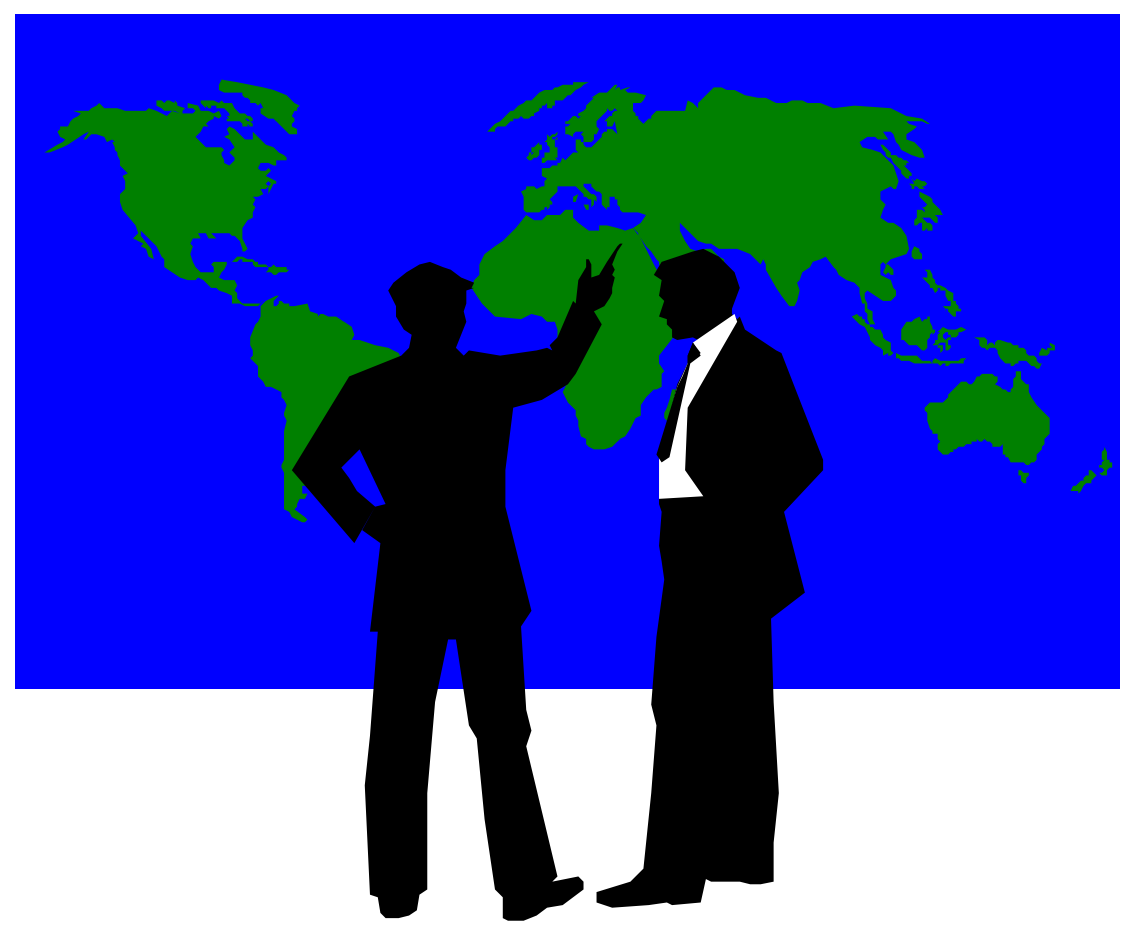

Fig. 30. Situational Awareness.

\section{Funding}

Internal - Laboratory Director's Research and Development

\section{Status/Maturity}

Conceptional 


\section{PACIFIC NORTHWEST NATIONAL LABORATORY}

\subsection{UAV SURVEY SYSTEM (USS)}

\section{Technical Contact}

Gerald A. Sandness, PNNL

Phone: (509) 375-3808

Fax: (509) 372-4725

E-mail: ga_sandness@pnl.gov

\section{Description of Technology}

The USS is a low-altitude aerial system for detecting buried manmade objects. The system uses the Cypher Unmanned Aerial Vehicle (UAV) as a platform for a variety of sensors. The Cypher is a unique, small, donut-shaped, rotary-wing, unmanned aircraft (see figure) that is being developed by Sikorsky Aircraft (Stratford, CT), a major manufacturer of military and commercial helicopters. The original purpose of this aircraft was to perform military reconnaissance functions. During the past two years, PNNL has worked with Sikorsky to optimize the aircraft for geophysical sensing applications, mainly the detection of underground structures. However, it has also proven to be capable of detecting smaller buried objects such as UXO.

The performance features and mechanical characteristics of the Cypher make it well suited to perform precise, low-altitude, airborne surveys with geophysical sensors such as magnetometers, ground-penetrating radar, thermal IR imagers, and electromagnetic induction devices. In particular, it is an exceptionally safe aircraft to operate because its two coaxial counter-rotating blades are shrouded to protect ground personnel. The aircraft is approximately $2 \mathrm{~m}$ in diameter, weighs approximately $250 \mathrm{lbs}$, and can fly at speeds between 0 and $30 \mathrm{kt}$. Its current payload is approximately $30 \mathrm{lb}$. The fuselage and other components are constructed primarily of non-metallic materials to minimize weight and to minimize the radar cross section of the aircraft. The non-metallic structure also ensures that the magnetic field perturbations produced by the aircraft are relatively small. The ability to fly as low as $2 \mathrm{~m}$ above the ground permits high-sensitivity detection of small buried targets.

\section{Status}

The development of the aircraft has been funded by Sikorsky. The adaptation of the aircraft for use as a sensor platform is currently supported by DOE's Office of Nonproliferation and National Security. The aircraft that is currently being flown is a technology demonstrator which does not yet exhibit the performance characteristics that will be provided by later operational versions of the aircraft. Nevertheless, in field tests at Sikorsky's flight test facility and at the Nevada Test Site, it has been demonstrated that the aircraft/sensor system can provide high-speed areal coverage, a high level of personnel safety, and good detection sensitivity for buried targets. The development of the Cypher is continuing. Additional work is needed to further improve the sensitivity of existing magnetic and EMI sensors and to interface and test new sensors. The system can be scheduled for tests and demonstrations. 


\subsection{REMOTE CHARACTERIZATION SYSTEM (RCS)}

\section{Technical Contact}

Gerald A. Sandness, PNNL

Phone: (509) 375-3808

Fax: (509) 372-4725

E-mail: ga_sandness@pnl.gov

\section{Description of Technology}

The RCS uses a small remotely controlled ground vehicle as a platform for a variety of non-intrusive sensors that can detect and map mines, unexploded ordnance (UXO), and other types of buried waste materials. The system was designed to perform large-scale site characterization surveys using telerobotics to minimize risk to personnel and to offer a high level of efficiency and cost effectiveness. The main components of the RCS are: 1) a small, self-propelled, remotely controlled survey vehicle (see figure); 2) a trailer for transporting the survey vehicle; 3 ) a truck-mounted control station (or base station) that is normally located outside the survey area; 4) subsystems for telemetry and navigation; and 5) a set of sensors for target detection.

The LSV is based on a six-wheeled design with modified skid steering. It is approximately $7 \mathrm{ft}$ long and $5 \mathrm{ft}$ wide and weighs approximately $800 \mathrm{lbs}$, including a payload of approximately $150 \mathrm{lbs}$. The chassis is articulated to minimize the vertical movement of the instrument platform in response to the roughness of the ground surface. The materials and components used in the construction of the survey vehicle were largely non-metallic to minimize the effects of the vehicle on the responses of the sensors.

The following sensors have been successfully operated on the LSV:

- Cesium vapor magnetometers

- Fluxgate magnetic gradiometers

- Ground-penetrating radar

- Metal detector

- Sodium iodide gamma spectrometer

\section{Status}

The existing prototype was constructed in the 1991-1993 period as a collaborative effort involving PNNL, INEL, SNL, ORNL, and LLNL. The development effort was jointly supported by the Department of Energy's Office of Technology Development and the U. S. Army Environmental Center. The current system was tested at INEL's Cold Test Pit and was recently used at the Aberdeen Proving Ground to survey a 170-acre UXO site. It is available for additional field tests and demonstrations. The existing technology and the lessons learned provide a basis for the construction of fully operational mine and UXO detection systems.

Next-generation survey vehicles that are smaller, lighter, and less costly can be constructed. Funding level for development was approximately $\$ 3 \mathrm{M}$.

\subsection{NUCLEAR METHODS FOR DETECTION OF MINES AND UNEXPLODED ORDNANCE}

\section{Technical Contacts}

David E. Robertson

Phone: (509) 376-5664 
Fax: (509) 376-3868

E-mail: DE_Robertson@PNL.gov

Ron L. Brodzinski

Phone: (509) 376-3529

Fax: (509) 372-0276

E-mail: RL_Brodzinski@PNL.gov

Richard J. Arthur

Phone: (509) 376-0405

Fax: (509) 372-0276

E-mail: RJ_Arthur@PNL.gov

\section{Description of Technology}

The Nuclear Chemistry Group of the Pacific Northwest National Laboratory (PNNL) has extensive experience and capabilities in nuclear technologies that are directly applicable to the detection of mines and unexploded ordnance. The two primary nuclear technologies having this capability are instrumental neutron activation (INAA) and x-ray fluorescense (XRE7) analyses. Nuclear Chemistry Group staff have many years of experience in these two areas, developing and applying advanced nuclear instrumentation for a wide variety of environmental, materials characterization, and safeguards monitoring needs.

Recent projects have included the development and testing of a portable, fieldable XRF analyzer for performing down-hole I" situ elemental analysis of soils at contaminated field sites and waste disposal trenches. This project was funded by DOE/OTD. An earlier XRF instrument was developed under DOE support to perform in situ elemental analyses of ocean and lake sediments. This system could be lowered to the ocean floor where the XRF analysis was performed and the analytical data was transmitted to a computer onboard the ship. In addition to these portable, fieldable XRF instruments the Nuclear Chemistry Group has considerable expertise and experience in the design, development and testing of laboratory XRF analyzers.

Nuclear Chemistry Group staff also have many years of experience in performing INAA of a wide variety of materials. The group maintains and operates a wide variety of state-of-the-art gamma spectrometers which are used for projects requiring INAA capability. Neutron irradiations of sample materials can be performed in a special sub-critical Cf-252 fueled, U-235 enhanced neutron multiplier facility located in the 329 Building. Staff also use the research reactors at Washington State University and Oregon State University for performing neutron irradiations for INAA. Portable, fieldable INAA instruments, based on Cf-252 neutron activation, have been developed and tested for conducting elemental measurements of ocean sediments and for the sub-surface detection of gold and silver bullion from 17th and 18th century shipwrecks buried in ocean sediments. These projects were developed under DOE and private funding sources.

The XRF and INAA technology developed and perfected at PNNL can readily be adapted to the detection and measurement of subsurface mines and unexploded ordnance. The necessary staff, expertise, experience, and facilities are in-place and readily deployable for immediate application to this important need.

\subsection{ULTRA-SELECTIVE VAPOR "SNIFFER" FOR GAS PHASE CHEMICAL MEASUREMENTS}

\section{Technical Contacts}

Stephen W. Sharpe

James F. Kelly

John S. Hartman 
Phone: (509) 375-5942

Fax: (509) 375-2799

E-mail: sw_sharpe@pnl.gov
(509) $375-2699$

(509) 375-2799

jf_kelly@pnl.gov
(509) $375-2771$

(509) 375-6736

js_hartman@pnl.gov

\section{Capability Summary}

An optical based instrument for real-time, ultra-selective and ultra-sensitive detection of targeted vapor phase chemical analyses has been demonstrated in the laboratory. This adaptable chemical sensor (the "Sniffer") can generally provide analyte concentration measurements at sub-parts-per-billion (ppb) sensitivity with direct air sampling in $\sim 1$ second total analysis times. The instrument can identify nearly any preselected volatilizable molecular or atomic species that exhibits a sharp absorption or dispersion feature in the infrared (IR) spectrum covering 0.65 to $12 \mu \mathrm{m}$. Its sensitivity and extraordinary dynamic range ( $>106: 1$ ) permit very precise distributed isotopic abundance measurements to be made on analytes (e.g., ambient trace atmospheric gases such as N20 at $<300 \mathrm{ppb}$ ). The analyzer embodies simple flexibility and rapid absolute abundance analysis without any sample calibrations or pretreatments.

\section{Technology Description}

The instrument is based on the absorption of a narrow-band, variable wavelength optical beam by gas phase molecules to detect and quantify the concentration of pre-selected gas phase analytes. The instrument concept is very simple. Low-power laser diodes are used to measure absolute species concentration by absorption. The gas or volatile vapor is admitted into a low pressure ( 30 Torr) sampling chamber that provides a folded optical path with long effective optical path length in a relatively small chamber. Alternatively, hollow-core optical waveguides can be employed if advantageous for the application. The most sensitive region of detection for most molecules is the IR spectrum and this will currently require that the laser diodes be cryogenically cooled (coverage of 3 to $12 \mu \mathrm{m}$ can be done with cooling in the range of 120 to $77 \mathrm{~K}$ ). In addition, the IR detectors also need to be cooled for best performance, but sensitive quantum detectors are available that now work well to $120 \mathrm{~K}$. Taken together, these cooling requirements are consistent with the capabilities of new portable mechanical cryo-coolers.

High spectral selectivity is achieved, in part because the thermal Doppler widths of the absorption features is smaller than in the ultraviolet and visible spectral regions, and because operation at low base pressures assure Doppler resolved lines devoid of atmospheric collisional broadening. Operation under these conditions does not diminish the peak absorbance sensitivity as explained by Ladenburg's principle of Doppler-resolved absorbance measurements. Thus high spectral specificity and sensitivity are attained simultaneously under the selected operating conditions. This allows excellent analyte isotopic constituent discrimination when working in the fingerprint region of the IR spectrum ( 3 to $20 \mu \mathrm{m}$ ) almost all molecules exhibit superb, uniquely distinctive spectra with excellent isotopic discrimination. In general, we have found dominant atmospheric IR active gases and water-vapor not to be a problem with our bench-scale system.

\section{Technical Status}

A laboratory instrument system has been developed and demonstrated with a variety target analytes. The instrument is available to support studies for LM/UXO applications.

Complementary PNNL instrumentation is available to perform the high resolution gas phase analyte IR spectral characterization required to determine and select spectral absorption peaks that will allow unambiguous measurement of target analytes without confounding effects of possible interferents. 
Concepts have been developed and proposal or pending for extending the instrument design to field portable configurations.

\subsection{ARTIFICIAL NEURAL NETWORKS}

\section{Technical Contact}

Lars J. Kangas

Phone: (509) 375-3905

Fax: (509) 375-6631

E-mail: lj_kangas@pnl.gov

\section{Description of Technology}

An artificial neural network (ANN) is an algorithmic system implemented in either software or hardware. The concept of ANNs was inspired by the way the biological brain processes information. ANNs, like people, learn by example. Learning in the biological brain occurs in a network of neurons that are interconnected by axons. A point of contact (actually most often a narrow gap) between an axon from one neuron to another is called a synapse. Learning is a matter of adjusting the electrochemical connectivity across the synapses.

An ANN is a network of neurons or processing elements (PE) and weighted connections. The connections correspond to axons and the weights to synapses in the biological brain. A PE performs two functions. It sums the inputs from several incoming connections and then applies a transfer function to the sum. The resulting value is propagated through outgoing connections to other PEs. Typically, these PEs are arranged in layers; with the input layer receiving inputs from the real-world and each succeeding layer receiving weighted outputs from the preceding layer as its input. Hence the creation of a feed forward ANN in which each input is fed forward to its succeeding layer. The first and last layers in this ANN configuration are typically referred to as input and output layers. (Input-layer PEs are not true PEs in that they do not perform a computation on the input). Any layers between the input and output layers (usually 0-2 in number) are called hidden layers because they do not contact with any real-world input or output data.

Back propagation is one of several possible learning rules to adjust the connection weights during supervised learning (learning by example). Learning occurs when the network weights are adjusted as a function of the error found in the output of the network. The error is the difference between the expected output and the actual output. The weights are adjusted backwards (back-propagated) through the ANNs network until the error is minimized for a set of training data. Another type of ANNs uses unsupervised learning. These ANNs cluster the data according to inherent differences in the data, i.e., these clusters are not influenced by predetermined categories of the data.

ANNs have been applied to an increasing number of real-world problems of considerable complexity. Their most important advantage is in solving problems that are too complex for conventional technologies; that is, problems that do not have an algorithmic solution or for which an algorithmic solution is too complex to be found. In general, because of their abstraction from the biological brain, ANNs are well suited to problems that people are good at solving, but for which computers are not. These problems include pattern recognition, modeling, and forecasting.

\section{Technical Status}

Ground-Penetrating Radar Target Signature Analysis Project 
- This project developed an artificial neural network based proof of concept software system to identify targets in ground penetrating radar signals. This project was completed for a non-DOE sponsor in 1992.

Other projects, past and present, at PNNL that use artificial neural networks in data analysis

- Modeling and Diagnosing the Cardiovascular System

- EEG Analysis in Anesthesiology

- Radiology Image Analysis

- Fetal Imaging

- Diagnosing Gas Turbine Engine Fuel Faults

- Circulation Pump Diagnostics

- Chemical Sensor Data Analysis

- Graph Data Extraction

- Power Load Forecasting

- Speech Recognition / Speaker Identification

- Acoustic Emission Signal Analysis

- Laser Range Imaging

- Nuclear Physics Mass Modeling

- Alpha Spectral Analysis

- Gamma Spectral Analysis

\subsection{GENETIC ENGINEERING OF ORGANISMS FOR HIGHLY SELECTIVE DETECTION OF MINES AND UNEXPLODED ORDNANCE}

\section{Technical Contact}

Dr. Peter C. Rieke

Phone: (509) 375-2833

Fax: (509) 375-2186

E-mail: pc_rieke@pnl.gov

\section{Objective}

The objective of this work is to demonstrate the feasibility of a sensor system for mines and unexploded ordnance (UXO) based upon organisms that metabolize explosives. We will demonstrate that this approach results in high specificity for this family of analytes. Specifically we will focus on the genetic engineering aspects and the analytical scheme necessary to produce reliable quantifiable detection. The result will be a genetically engineered organism that can be used in the flow injection analysis scheme to analyze for explosive compounds. 


\section{Technical Approach}

Our approach is to combine relevant aspects of bioremediation, molecular biology and analytical chemistry to develop a general strategy for design of highly selective sensors for mines and UXO. We utilize the inherent ability of bacterial organisms to evolve gene regulation chemistries in response to the presence of toxic or harmful environments. The approach consists of three technical steps: 1) identify and characterize an organism that degrades a specific analyte, 2) insert a reporter gene into the organism that results in a detectable analytical signal, 3) utilize flow injection analysis to optimize the analytical scheme and obtain quantitative data. The proposed work will focus on the latter two steps. We will make use of recent work on the identification of organisms that degrade various explosive compounds.

The engineered organism provides the basis for sensors but also provide a means of understanding the relevant metabolic pathways associated with analyte degradation. Pathway studies can be utilized to identify key proteins and enzymes associated with the gene regulation process. The isolated compounds may then be used for analyte recognition ex situ and incorporated into more conventional sensor platforms such as electrochemical, waveguide, and piezoelectric transducer platforms.

\section{Technical Status/Maturity}

The research is currently funded (FY96) through internal LDRD lab funding at 140K/year. We expect to obtain additional LDRD funding for FY97. The focus of this research has been on developing the overall protocol for the necessary genetic engineering and analytical scheme. The analytical equipment is set up and available for laboratory demonstration. The genetic engineering laboratory is also available for tours although is not specific to this project. Current efforts are on development of sensors for polychlorinated phenols and nitrilotriacetic acid based on organism used in bioremediation of the Hanford engineering site.

\subsection{THE DETECTION OF MINES AND UNEXPLODED ORDNANCE (UXO) VAPORS AND PARTICULATES USING AN INTEGRATED MICROTECHNOLOGY PRECONCENTRATOR AND SENSOR SYSTEM}

\section{Description of Technology}

The Land Mind (LM) and Unexploded Ordnance (UXO) detection technology embodied within the product delivered at the conclusion of this project will dramatically advance the state of the art in total detection system miniaturization. Our approach will utilize a novel mesoscale architecture technology. This technology will reduce the size and weight of the proposed detection systems and create a standardized platform for the incorporation of emerging third party technologies, as well as technological upgrades. The core of the system will be the innovative collection, adsorption and preconcentration of target vapors in our microchannel heat exchange/HEPA filtration device. This unique collection/ filtration system will be coupled to three distinct detection systems: 1) a micro machined Gas Chromatograph (GC) with an embedded electron capture device, 2) an Atmospheric Ionization Source coupled with a Time of Flight Mass Spectrometer AI-TOFMS), and 3) a bioluminescence detector (BD) with immobilized enzymes in a microchannel device. The completed system will be suitable for hand held field operations. A proof-of-concept demonstration will be performed prior to the conclusion of this 3-year project. 


\section{Innovative Claims of the Proposed Technology}

The technology developed will have the following features which advance LM/UXO detection system technology to a level that equal to the threat confronting both the war fighter and the humanitarian relief provider.

1. This detection approach lays the foundation for an overall system design that incorporates several innovative and novel concepts to advance LM/UXO-explosives detection. The system will provide a universal air collection, filtration, concentration, and handling system that can support a wide variety of detection systems. The air collection system will collect chemical vapors, aerosols, and particulates by employing a microchannel heat exchanger packed with a thermally regenerable adsorbent to rapidly concentrate samples for an array of detectors. This microchannel heat exchanger has been demonstrated to quickly heat and cool samples every few seconds in a biological detection system. The microchannel heat exchanger will be coated to prevent the degradation of the explosive materials due to contact with a hot metal surface. At the end of each microchannel, the sample will be delivered to a different detector, thus allowing our approach to incorporate a variety of detectors to maximize the probability of detection.

2. We will develop a Micro machined gas chromatograph with miniaturized electron capture detector. This miniaturized detection device will be capable of detecting target compounds at the low ppb/ppt range.

3. We will develop a miniaturized Atmospheric ionization source coupled with a time-of-flight spectrometer that will also exhibit similar detection sensitivity as well as the capability of discerning the target compounds from any possible trace interferrents resident within the sample stream.

4. We will also miniaturize and incorporate a microchannel system impregnated with immobilized enzymes to create a Bioluminescence detection strategy that is specific for the target compounds. Our three detector strategy is designed to take advantage of the strengths of each systems, and blend them together to provide a sensitive detection system that is robust enough for continuous field operations.

\section{Technical Approach and Rationale}

The need clearly exists for the LM/UXO/Explosive detection systems which are small in size, have minimal false alarms, and yet respond quickly. Minimization of false alarms can best be achieved through the incorporation of multiple sensor technologies into a single detection system. The development of our detection system is broken down into three primary tasks: 1) development of the mesoscale motherboard that will incorporate the collection and detection sub-systems, 2) air collection and preconcentration of the target compounds, 3) target compound identification. Integrated performance of these functions requires a control system that can optimize power consumption and consumables.

\section{Mesofludics System Integration Motherboard}

The mesoscale system platform is an architecture which allows the compact integration of mini, meso, and microscale electronic and sample processing components. This is accomplished through an extension of the concept of a personal computer motherboard to include the channels for sample transfer connections as well as electronic connections. This approach facilitates the development of a rugged platform to attach mini and microelectronics, as well as fans and heat sinks; it facilitates multiple layers of electrical connections between mini-electronics and microelectronics; and has low manufacturing costs. In addition to these functionalities, the mesoscale design allows for the transfer of either gaseous or fluid based samples to be transported to the detectors. This is accomplished through a multi layer motherboard. The bottom layer is the conventional fiberglass motherboard with copper traces for electronic connections. The middle layer is comprised of 
Teflon or another suitable polymer with etched channels for either fluid or gaseous transport, and a top polymer layer will seal the channels. The motherboard will be designed from the ground up to be capable of incorporating our and other third party detection systems. The project will work with a cross-section of the user community to ensure that user requirements are fully incorporated in the development of the standard. The standard will address such issues as: electrical power requirements, signal input and output, consumables and sample delivery, physical footprint, profile and weight. The product delivered at the end of this project will allow "plug and play" modularity.

\section{Sample Collector, Preconcentrator and Transport System}

The sample collector/Preconcentrator will collect vapors, aerosols, and Particulates by using a microchannel heat exchanger packed with a thermally regenerable adsorbent protected from gross contamination by a thermally regenerable ceramic HEPA filter. The ceramic HEPA filter will be capable of being heated to volatilize adsorbed target compounds for introduction in the detection scheme. The ceramic HEPA will be able to be cleaned by simply reversing the air flow and thereby expelling any adsorbed materials. The microchannel heat exchanger will be coated to prevent the degradation of heat sensitive target compounds from coming into direct contact with the heat surface. The preconcentration component is composed of two parts; a particulate and aerosol filtering stage followed by the vapor concentration on an adsorbent matrix. We assume that the sample gas stream will contain a mixture of gases and Particulates, as well as other interferrents. The first part of the sample preparation will result in the capture of the particulate. This aerosol removal step is accomplished at the distal portion of the microchannel heat exchanger through a ceramic High Efficiency Particulate Air (HEPA) filter. This fibrous filter will efficiently collect Particulates while the mixtures of explosive vapors continue down the microchannel. The advantage of this approach is that the HEPA can be heated to desorb and remove Particulates, thus creating a self-cleaning filtration system. The cleaning can be done while the filter is still in place by reversing the air flow, greatly simplifying maintenance.

The microchannel heat exchanger itself will serve to concentrate the explosive vapors before introducing them into the detectors. Our planar sheet architecture device provides micro channels (which are a micron deep with a small gap distance and a few centimeters long) filled with adsorbent. This concentration step is required to the low vapor pressure of the explosives. The coated microchannel heat exchanger will be packed with an adsorbent material, such as Tenax which can be thermally regenerated, thereby reducing down time due to maintenance. Usually a vapor concentrator will increase the amount of time required to complete an analysis. However, mass (and heat) transport resistances are significantly reduced within micro channels; for example, for each order of magnitude reduction in transport pathway length, molecular diffusion time reduces by two orders of magnitude. (We have verified the feasibility of placing catalytic adsorbents in a microchannel and heating quickly for another DARPA project).

\section{Micro Machined Gas Chromatograph With Electron Capture Detector (ECD)}

The detection of explosives with the electron capture detector (ECD) with a gas chromatographic column has been demonstrated at the ppt level. The ECD is a very sensitive detector for molecules containing electronegative atoms such as halogen or nitrogen atoms. A gas whose molecules exhibit electron affinity or an ability to pick up free electrons or for negative ions, will be sensed. Our ECD will be equipped with a ${ }^{3} \mathrm{H}$ source. This sensor will require the use of a carrier gas of $5 \% \mathrm{CH}_{4}$ in argon or nitrogen.

The micromachined gas chromatograph would accept the desorbed sample into the miniaturized parallel capillary columns commercially available from Alltech, Inc. The separation time is expected in the seconds range. The system is shown schematically in Figure 1 with the integrated packed microchannel heat exchanger. 


\section{Atmospheric Pressure Ionization Source With A Time-Of-Flight Mass Spectrometer}

Another microchannel will send its flow to the atmospheric ionization/time-of-flight mass spectrometer. We will fabricate and test a novel approach for detecting chemicals using a unique configuration of a miniaturized plasma reactor integrated with a Herriott optical cell (MPRHC). This miniaturized MPRHC configuration can uniquely and rapidly identify chemicals by the combination of analytical methods in a single device; the emission and absorption of light within a plasma and absorption of light along a long optical path (using wavelength modulation spectroscopy). This device enhances the state-of-the-art of field detection methods through its ability to rapidly identify and quantify specific contaminants and intermediates.

Long optical path lengths are required for this system to perform adequately. Suitable optical path lengths (approximately $10 \mathrm{~m}$ ) are available from compact, Herriott-style multiple pass cells. These cells use a pair of curved (focusing) mirrors to provide extended optical paths within a small structure. The input laser beam is collimated by a Grin (graded index) lens at the output end of the optical fiber and enters the cell through a hole drilled near the edge of one mirror. The beam bounces back and forth between the two mirrors and is refocused on each pass. This constant reimaging keeps the optical trajectory stable despite vibrations and torsions of the cell. After a selected number of passes, the beam impinges on a fiber optic which leads to the detector. Our approach takes advantage of the fact that reflected beams within the Herriott cell strike the mirrors near the outer rims; the central region is not used. Therefore, we plan to use annular mirrors. The central openings in the Herriott cell permit the integration of the high voltage electrode creating a plasma (or ionized gas). This MPRHC design combines three measurement techniques in a single device including emission spectroscopy, absorption spectroscopy of a plasma and gas environments, creating an accurate, fast, and sensitive sensor. The device is expected to be reliable, low power consumption, compact design, and zero maintenance. The reduction in the mass spectrometer size is a significant technical challenge. TOFMS instruments rely on linear distance to separate components. As such, there may be a fundamental limit as to the combination of ion acceleration and linear distance required to achieve separation.

\section{Bioluminescence of Immobilized Enzymes in a Microchannel}

It has been shown that a system for the detection of TNT utilized an enzyme that catalyzed the reduction of TNT molecules. That system was highly specific for TNT and could detect $1 \mathrm{ppt}$ and we believe that if this occurred in a microchannel the analysis period would be a few seconds long. In general, the bioluminescent signal was obtained by a series of chemical reactions occurring in the detector module. Two primary reactions can occur: one with the TNT absent and the other with TNT present. In the absence of TNT, $\mathrm{NADH}$ (reduced form of nicotinamide adenine dinucleotide) produced a reduction product, $\mathrm{FMNH}_{2}$, which then underwent a bioluminescent reaction, the intensity of which is measured by a photo multiplier. When TNT was present, the TNT molecules were enzymatically reduced by the TNT enzyme, also consuming NADH molecules. This competitive reduction led to subsequent decrease in the intensity of the bioluminescence, as measured by the photo multiplier. The inclusion of enzymes within micro channels will provide the ability to carry out in an efficient manner due to the high turn over number, high specificity, and high affinity of enzymes. The enzymatic component provides the

high specificity and rates; the electrochemical component provides an economical source/sink of electrons, and the means to monitor reactants and products; and micro technology provides high surface area per unit volume for the reaction to occur. 


\subsection{UWB RADAR HOLOGRAPHIC IMAGING SYSTEM FOR BURIED METAL AND PLASTIC MINES}

The proposal hand-held imaging system (HIS) will use a small rotating linear array located in a metal detectorshaped device as demonstrated below. It has a rotating tapered-slot array which is housed in a head of the system and the image seen by the operator. The operator wears virtual vision glasses which allow him to see the actual ground surface in one eye and the subsurface image in the other eye for instant detection and identification. Ultra wide band (UWB) holographic imaging technology developed over the past several years at PNNL will be utilized in building the hand-held system; significantly reducing the time an cost for the first field system.

The objective of this project is the development of a hand-held, UWB holographic camera, capable of imaging shallow subsurface plastic and metallic mines with extremely high image resolution ( 1 to $2 \mathrm{~cm})$. The proposed UWB radar holographic imaging array system will be constructed using proven radar subsurface technology that was developed by Pacific Northwest National Laboratory (PNNL) for the Department of Energy (DOE) for imaging buried waste at their various sites. The UWB radar system uses a long linear array (tapered-slots) mounted on a vehicle for real time imaging of buried waste at various DOE sites.

UWB radar holography is a unique technique for imaging subsurface targets such as mines, unexploded ordnance (UXO), etc., with extremely high depth and lateral resolution. Recent sweep frequency (12 to $18 \mathrm{Ghz}$ ) holographic images of simulated mines (plastic \& metal), letter " $F$ " and a small sphere (-30 dbsm) buried at 15 $\mathrm{cm}(6 ")$ depth in sand graphically illustrate the resolution capabilities of the UWB subsurface holography.

The images and geometry of four simulated mines, two plastic (dielectric) and two metal can illustrate the excellent image resolution of the PNNL real-time array holographic system. The metal and plastic mines, which vary in size from about $5 \mathrm{~cm}$ to $7.5 \mathrm{~cm}$, are easily identified in the image. The bright images are metal mines with high reflectivity to radar energy and the less bright images are plastic mines with low reflectivity.

The latest subsurface systems developed at PNNL also present a side, or depth view, and an end view for exact depth location.

The image of the metal letter " $F$ " and a small sphere buried $15 \mathrm{~cm}$ in sand have been detected. The $4 \mathrm{~cm}$ diameter sphere has a radar cross section of approximately $30 \mathrm{dbsm}$ and can be easily seen. It is very significant for a ground penetrating radar system with sufficient dynamic range and signal-to-noise ratio to detect and image a buried target with this small radar cross section. The width of the "F" is typically two wave lengths $(4 \mathrm{~cm})$ at $15 \mathrm{Ghz}$ in air and are resolved in the image with excellent lateral resolution. The green circular to the left of the " $F$ " is an image of the small sphere. The color green represents small reflected amplitudes in the image (i.e., typically $-15 \mathrm{db}$ below the letter "F").

Four metal mines (simulated) of various sizes $(2.5 \mathrm{~cm}$ to $13 \mathrm{~cm}$ ) buried $15 \mathrm{~cm}$ in sand using a sweep frequency system operating between 12 and $18 \mathrm{Ghz}$ have been detected. The small $2.5 \mathrm{~cm}(1 ")$ mine is easily seen and identified illustrating the excellent resolution of the UWB holographic system.

Previous research and development efforts in subsurface imaging have proven the viability of UWB holographic "3-D" imaging. The near real-time imaging system we recommend is similar in shape and size to a metal detector (Figure 1). This unique radar system, with a rotary linear array in the circular head, is an extremely cost effective solution for high resolution imaging of UXO in " 3 -D". The design, which is extremely simple and rugged, provides essential real-time imaging from $8 \mathrm{Ghz}$ to $14 \mathrm{Ghz}$ by employing an integrated rotational multi-element tapered-slot array generating approximately 3000 image points (for each depth) in one-half disk rotation. 
The holographic array consists of 32 antenna elements (16 transmit and 16 receive) with center-to-center spacing of approximately one wave length $(2.5 \mathrm{~cm})$ at $12 \mathrm{Ghz}$.

PNNL has developed a sweep frequency radar front-end and bi-static antenna configuration which significantly reduces the front-surface reflection and thus increases the dynamic range, etc. This dramatically improves the system's ability to image buried targets with high signal-to-noise ratios compared with conventional impulse systems.

The UWB hand-held radar holographic mine imaging system will be light weight, portable, battery operated with a backpack computer/image processor, and virtual vision glasses for field operation. The operator wearing small virtual vision glasses will view subsurface UXO images in real-time as he scans the terrain.

The major objective of the UWB radar technology is the development of a portable hand-held ultra-wide (UWB) holographic system, capable of imaging shallow subsurface UXO with high depth and lateral resolution $(1$ to $3 \mathrm{~cm})$ in real time.

The system will be similar in size and shape to the familiar metal detector with a circular head. The operator will scan the radar array head back and forth over buried UXO and view the TV image with virtual vision glasses. Mines will be accurately identified by their unique image in "3-D" and not by signal identification which is the most common conventional technique.

A small backpack computer with associated battery pack will process the data in real-time for instant detection and identification of plastic and metal UXO of all sizes.

The Acoustics and Electromagnetic Imaging Group at PNNL has developed and delivered numerous single frequency and UWB radar holographic imaging systems to various government agencies. These systems range from portable hand-held "2-D" and rotary linear array UWB holographic cameras, to trailer mounted subsurface holographic imaging systems, to 20 meter long near-field holographic RCS measurement and imaging systems. The staff identified in this proposal each have 10 to 20 years of experience in design, fabrication and testing of holographic imaging systems, which include state-of-the-art software designed to make each system user-friendly and easy to maintain in the field.

\title{
8.9 Detection Of Land Mines And Unexploded Ordinance Using An Enzymatic Chemical Reaction Based Sensor
}

\section{Technical Contacts}

\author{
C. L. Shepard \\ B. J. Burghard \\ M. M. Shah
}

\section{Description of Technology}

The problem of detection of explosives has been attacked with various methods to date. Canines appear to be quite effective at the detection of explosives for reasons not entirely understood. However, their use in the field has at times demonstrated some limitations on reliability. We propose a method for detection of explosives which is based on detection of vapors generated and released by the explosives. The vapor 
pressures are quite low and thus an extremely sensitive instrument is necessary. We believe that the proposed instrument can duplicate the olfactory capabilities of canines.

\section{Proposed Solution}

Certain enzymes in the presence of explosives in molecular form react with the explosive and form a radical molecule. In the presence of oxygen, these radicals will generate hydrogen peroxide very efficiently. Hydrogen peroxide is a strong oxidizer which can be detected in minute amounts with electrochemical sensors. Our sensor system will take advantage of the above process.

A sample is drawn from the air and is passed through a charcoal filter which is partially immersed in a water solution containing an enzyme. The enzyme ferredoxin NADP oxidoreductase, referred to as FNR, has been shown to be effective for the proposed detection scheme and will be used in this work. Any explosives vapors, such as TNT, within the air sample are collected by the charcoal as air flows through it. The charcoal disks are actively rotated at a slow rate so that any collected explosives contact the liquid. Once in the liquid, the explosives can undergo the aforementioned enzymatic reaction with the resulting production of $\mathrm{H} 202$ which is detected by an electrochemical sensor also resident in the liquid bath. Several possibilities exist for choice of hydrogen peroxide sensor and we will determine the best choice after some initial study. The detection of $\mathrm{H} 202$ constitutes detection of explosive vapors. For TNT the reaction to produce $\mathrm{H} 202$ proceeds as follows:

$$
\begin{gathered}
\text { FNR } \\
\text { TNT } \stackrel{----->\text { [TNT]- }}{ } \\
{[\text { TNT]- + } 02 \text {------> H202 + TNT }}
\end{gathered}
$$

Note that TNT is not consumed by this process, only oxygen. This result is very favorable since the TNT will be in short supply. Reaction of TNT with the enzyme produces a TNT radical while reaction of the TNT radical with oxygen (dissolved in the water) restores the original TNT molecule with the generation of H202. Thus, a quantity of TNT in solution will produce H202 until the supply of oxygen is exhausted. The rate at which $\mathrm{H} 202$ is generated will depend on the amount of TNT held in the solution. The two-step reaction shown above occurs at a favorable rate. One nanomole of TNT can produce 140 nanomoles of $\mathrm{H} 202$ per minute for each milligram of enzyme. With a vapor phase concentration in air of 1 PPB an air sample of 0.24 liters needs to be collected in order to obtain 0.01 nanomole of TNT, assuming the charcoal collector is $100 \%$ efficient. This amount of air can be pumped through the collector within several seconds and is probably similar to the amount of air which a good dog might process in detecting TNT. With a liquid sample volume of about $10 \mathrm{~cm} 3$ we believe that 0.01 nanomole of TNT will result in $140 \mathrm{nM}$ liquid phase concentration of $\mathrm{H} 202$ per mg of enzyme to allow detection within several seconds. H202 is soluble in water and its concentration will build in time. Over a long enough time, even very small quantities of TNT can produce enough $\mathrm{H} 202$ to be detected. For TNT, the sampling and complete testing could be done within about 5 seconds. By increasing the amount of air and enzyme, the sensitivity and response time can be improved. For other explosives such as RDX this time may be considerably longer due to the reduced vapor pressures of these explosives. Laboratory scale experimentation will be necessary in order to determine how well the method will work with other explosives. Many of the more common explosives have the same chemical structure needed for the enzymatic reaction to occur.

The salient features of the proposed sensor include:

- Sensitive, Real Time Response to Presence of Explosives in Air Phase

- Weight $<2 \mathrm{lb}$ 
In summary, we believe we have described a sensor concept which is suitable for real time detection of vapors from explosives. It relies on a concentrator (the charcoal) and an enzymatic reaction which produces a signal (H202) without consumption of the explosive. The sensor system should be quite inexpensive and a prototype system could be developed and tested within two years. In addition to detection of unexploded ordnance and land mines the sensor may be applicable to airport security functions if the response time proves fast enough.

\subsection{SMART TERRAIN PC-BASED TERRAIN VISUALIZATION CAPABILITY}

\section{Technical Contacts}

Randy Woodson, Project Manager

Phone: (206) 967-8507

E-mail: r_woodson@pnl.gov

John Risch, Chief Scientist

Phone: (509) 372-6052

E-mail: js_risch@pnl.gov

\section{Description of Technology}

Pacific Northwest National Laboratory, in collaboration with US Army I CORP 201st MI BDE, is developing a low-cost, PC-based terrain visualization system intended to support the visualization requirements of forces operating on 21st Century digital battlefields. The system, called Smart Terrain, takes advantage of emerging high-performance PC-based 3D graphics acceleration hardware and an advanced software architecture to provide a terrain visualization capability comparable to that previously available only on expensive, dedicated graphics computers.

Smart Terrain enables the display and navigation of high-fidelity 3D terrain models in real-time. Terrain models can be 'draped' with a variety of raster data types, including multiresolution overhead imagery and DMA ADRG maps. Smart Terrain v. 2.0, now under development, will provide the ability to incorporate local, high-resolution image 'insets' from sources such as NTM or aerial platforms. Version 2.0 will also support draping of vector information (e.g. control measures or political boundaries) onto terrain models.

The system uses COTS Pentium-level PC hardware running under Windows NT, avoiding the need for "swivel-chair" integration visualization capabilities with existing PC-based applications. An easy-to-learn userinterface and help system minimizes training requirements. Smart Terrain provides an inexpensive, generic, 3-D geospatial data visualization capability suitable for a wide range of applications.

\section{Base System Hardware Configuration}

200 MHz Pentium Pro CPU

3D Graphics Accelerator

64 M RAM

2 GB Hard Drive

CDROM Drive

\section{Total Hardware Cost}

\section{$\$ 12 \mathrm{k}$}




\section{Example Applications}

Mission Planning and Rehearsal

Intelligence Preparation of the Battlefield

Tactical Information Visualization

Environmental Data Visualization

Training

\section{Key Features}

Real-Time Interactive Terrain Flyovers

Advanced Terrain Modeling System

Moving-Map Display

View Position/Bearing/Elevation Readouts

Raster and Vector Data Overlays

Realistic Solar/Lunar Illumination

Applications Programming Interface 


\section{SANDIA NATIONAL LABORATORY}

\subsection{X-RAY SOURCES AND SENSORS FOR COUNTERMINE-LAND}

\section{Technical Contact}

Steven Shope

Sandia National Laboratories

Org. 1221

Albuquerque, NM

Phone: (505) 845-7659

E-mail: slshope@sandia.gov

\section{Description of Technology}

The Compton backscatter imaging technique has been shown in the laboratory to be an effective way to detect buried or hidden nonmetallic mines. This technique relies on the absorption and scattering of energetic photons. A narrow beam of photons is directed into the soil in which a mine may or may not be present. The probability that a photon will be absorbed or scattered is a function of the energy of the photon and the properties of the materials in which it interacts. The differences between mine and soil that allow this technique to work are the higher absorptive and lower scattering properties of soil compared to the nonmetallic mine. A unique detector system located above the soil intercepts backscattered photons. A higher detector response is recorded when the beam strikes the mine, than when it strikes only soil. Other materials in the soil such as organic matter may have photon interaction properties similar to the nonmetallic mine. To avoid false alarms from these materials, the beam is scanned over the area of interest and the detector response is recorded as a function of the position that the beam strikes the ground. The result is an image of the buried mine--the shape of which can be used to reduce false alarms. The differences in the absorptive properties of mine and soil provide the contrast for imaging. The detector output is transformed into an image by a microprocessor. Imaging algorithms developed at the University of Florida and unique detector arrangements allow surface features and clutter to be removed. This leaves an image of a buried land mine. Both metallic and nonmetallic mines have been successfully imaged in the laboratory under logs, rocks, water, snow, and other surface debris. The laboratory experiments have used a small, commercial medical x-ray machine.

\section{Application of Technology}

This technology is applicable to both military and civilian demining programs. In addition, it could be used in any application where transmission $\mathrm{x}$-rays are not practical as a suspect bomb in a container against a wall where $\mathrm{x}$-ray film cannot be safely placed around the object.

\section{Programmatics}

Sandia currently has a program with the Night Vision and Electronic Sensors Directorate at Fort Belvoir to field test a prototype backscattered mine detector in 1998. This program is jointly funded by the Army and the DoD/DOE Memorandum of Understanding. 


\title{
9.2 VERY LOW FREQUENCY SYNTHETIC APERTURE RADAR
}

\section{Technical Contact}

\author{
Dr. Paul D. Rockett \\ Sandia National Laboratories \\ Org 2527, \\ Albuquerque, NM \\ Phone: (505) 845-7466 \\ Fax: (505) 844-7020 \\ E-mail:pdrocke@sandia.gov
}

\section{Description of Technology}

Electromagnetic Induction (EMI) can locate small quantities of buried metal; however, the low frequencies involved result in virtually no directionality of this technique for mine detection. A loop antenna sends out an omnidirectional pulse of low frequency waves and a three-axis detector antenna records the return pulses from any metal in the area. Standoff detection with EMI is almost impossible. However, if the technique of synthetic apertures (as applied typically to radar) could be applied in this case to low frequencies (e.g. $500 \mathrm{~Hz}$ to $100 \mathrm{kHz}$ ), one may be able to obtain true direction information, i.e., just where and how deep is the sought after object? By collecting return signals over an extended distance using a pulsed radio frequency source, one may be able to construct a large synthetic aperture, capable of identifying the range and azimuth to locate the position of the underground metallic reflector.

\section{Application of Technology}

A helicopter or low flying aircraft could carry the pulse source and transmitting antenna as well as the collecting tri-axial antenna. (This, in fact, is a common geometry for EMI bathymetry over shallow waters.) The data, however, would be collected so that the detailed phase history of the return signal would be recorded as well as the amplitude. Because the transmitting antenna is omnidirectional, returns from an object will last for a long time as the airborne platform flies over the test field. Reconstruction of the phase data may enable the generation of a 2-D image, demonstrating the location of the returns and identifying the locations of UXO whether they are mines or unexploded shells. EMI sensitivity is high, so very little contained metal will be necessary for detection.

\section{Programmatics}

EMI techniques have been pursued at Sandia for the past three and a half years under DOE NN-20 sponsorship for the detection of underground structures. This technique has also been sponsored in the Tactical Underground Structures (TUGS) program under an Army MOU. In the former case, experiments successfully identified a tunnel and an underground building at the Nevada Test Site. The tunnel was $30 \mathrm{~m}$ deep and the building was $9 \mathrm{~m}$ deep. In the latter case, EMI was used for passive powerline detection to indicate the initiation of machinery at a distance, identifying the power turn-on signature for an air compressor and vibrator. EMI is a mature technology. However, the application of synthetic aperture imaging to such low frequencies and long wavelengths is quite new. 


\title{
9.3 HIGH PERFORMANCE CHEMICAL ANALYSIS SYSTEMS INTEGRATED ON A CHIP
}

\author{
Technical Contacts \\ Greg Frye \\ Sandia National Laboratories \\ Org. 1315 \\ Albuquerque, NM \\ Phone: (505) 844-0787 \\ Fax: (505) 844-1198 \\ E-mail: gcfrye@sandia.gov \\ Dave Rakestraw \\ Sandia National Laboratories \\ Org. 1315 \\ Albuquerque, NM \\ Phone: (510) 294-3297 \\ Fax: (510) 294-1004 \\ E-mail: drake@sandia.gov
}

\section{Description of Technology}

We are taking advantage of advances in microfabrication and in chemical analysis to develop a fully selfcontained, micro-ChemLab that can perform real-time detection of a wide range of chemicals with ultra high precision in the presence of complex backgrounds. This device, pocket calculator size or smaller, will contain both liquid-phase and gas-phase analysis channels. Each channel will contain three stages of separations/selectivity in order to attain ultrahigh sensitivity while still rejecting large complex backgrounds: selective preconcentration, chromatographic separation, and selective detection. Multiple parallel analyses channels will allow a single unit to detect a wide variety of chemical species while also providing multiple "independent looks" at the same species, thereby further improving accuracy. A complete analysis would be performed in approximately one minute. Drawing on much of the technology from the microelectronics world, these units should ultimately be amenable to high volume production at reasonably low cost. Additional work is focused on larger scale sampling systems that can collect explosives from large sample volumes and concentrate them into small gas or liquid samples for analysis by the micro-ChemLab as well as more conventional detectors.

\section{Application of Technology}

This micro-ChemLab technology will have the high performance required to detect trace levels of specific chemical signatures (e.g., explosives) in complex backgrounds (e.g., battlefield, sea water). This chemical information will be provided rapidly, enabling the system to be used to quickly verify that an object, identified by a metal detector or other nonspecific detector, does indeed contain an explosive. As the technology matures, it may be possible to use this capability to rapidly find and identify UXOs or mines, based on their chemical signatures. Because the system should be able to directly analyze species in both air and water samples, it could be used for both land-based and sea-based devices. The larger scale sampling systems being developed will be key to further enhancing the sensitivity of the micro-ChemLab technology for detection of very low explosive concentrations. These samplers could be used with more conventional detectors for nearer term (two year) capability demonstrations. 


\section{Programmatics}

Sandia, with its core competency in microelectronics and microphotonics and its excellence in systems integration and robotics, is ideally poised to make this total microanalytical system a reality. Microfabrication of integrated components and systems can be performed in house using two state-of-the-art facilities for microelectronics and micromachined systems. In addition, Sandia has extensive experience in (1) acoustic wave devices and optical detection systems for sensitive and selective chemical detection, (2) liquid and gasphase separations, and (3) sample collection and preconcentration. Beginning in OCT 1996, Sandia committed to a large internal LDRD investment to develop the enabling technologies to make a prototype micro-ChemLab system by SEP 1999. Some work on detecting key explosives (e.g., TNT, PETN, RDX) is planned for this development effort. The extensive ChemLab R\&D that is currently being done under the LDRD funding could be leveraged to optimize and extend it to UXO and mine detection applications and to move from a proof-of-concept prototype to a field hardened unit.

\subsection{SIGNAL AMPLIFICATION BY CHEMICAL REACTION}

\section{Technical Contact}

R. J. Simpson

Sandia National Laboratories

Org 2522

Albuquerque, NM

Phone: (505) 844-7599

E-mail: rjsimon@ sandia.gov

\section{Description of Technology}

We are investigating chemical reactions that will produce relatively large numbers of product molecules for each original reactant molecule.

\section{Application of Technology}

Several techniques exist for detection of vapors or traces of explosive or other target compounds characteristic of mines and unexploded ordnance. However, these techniques may not have sufficient sensitivity to detect mines or other targets under field conditions, using practical sampling techniques and measurement intervals. Use of the proposed chemical reactions would produce significantly higher concentrations of detectable analytes.

\section{Programmatics}

A three-year effort in this area is currently supported by DOE-sponsored internal funding at Sandia and through a DARPA-supported industrial partnership between Nomadics Corporation and Sandia.

\subsection{HAZARDOUS MATERIAL REMOVAL USING A HIGHLY DEXTEROUS, REMOTE MOBILE MANIPULATOR}

\section{Technical Contact}


Kwan S. Kwok

Sandia National Laboratories

Org 2111

Albuquerque, NM

Phone: (505) 845-7170 (voice and fax)

E-mail: kskwok@sandia.gov

Description of TechnologyA prototype mobile robotic manipulator called Remote TeleRobotic Vehicle for Intelligent Retrieval (RETRVIR) developed by Sandia provides a remotely piloted vehicle to safely conduct hazardous material handling activities such as removing unexploded ordnance. RETRVIR integrates multiple sensors, vision systems, and advanced onboard computing systems to allow remote operators to safely locate and manipulate hazardous material. RETRVIR consists of three major components: 1) a Honda all terrain vehicle to provide a highly mobile platform, 2) a Titan II 6 degrees-of-freedom (DOF) robotic manipulator to grasp and manipulate hazardous objects, and 3) a visual targeting system to locate objects positioned within the work space of the manipulator.

\section{Application of Technology}

RETRVIR provides an effective replacement for on-site human workers performing activities that typically require manual operations such as detecting and removing hazardous materials and toxic substances. The hazards associated with removing explosive material often prohibit workers from performing work in the immediate area without costly and cumbersome protective equipment. Many of the problems typically associated with humans performing manual tasks in hazardous environments can be mitigated by deploying remotely operated manipulators directly into the work area. Research activities at Sandia have demonstrated that remotely operated mobile manipulators significantly reduce the danger to human workers and improve the ability of a remote operator to safely handle hazardous objects from a safe distance.

RETRVIR's real-time sensing and high force manipulator capability enable controlled excavation of unexploded ordnance without requiring large scale excavation. RETRVIR has demonstrated effective waste retrieval operations at Sandia, Jefferson Proving Grounds in Madison, IN, and the Tonopah Test Range. During these system validation tests, RETRVIR successfully performed hazardous material removal in buried waste sites. More than 100 live and inert munitions were safely recovered at the Tonopah Range in a threeweek period. RETRVIR's combined automated and manual operations allow the system to quickly approach and grasp objects positioned on uneven terrain. The onboard vision system enables a remote operator to safely identify and grasp hazardous material using the hydraulically powered Titan II robotic manipulator. The remote camera system and high dexterity robot arm enable the operator to safely inspect objects and store them in onboard containers for transport to a local disposal facility.

\section{Programmatic Issues}

Employing human workers in hazardous object removal operations is experiencing increased scrutiny due to the high costs and low productivity associated with protective clothing and safety equipment. RETRVIR's work at the Jefferson Proving Grounds and the Tonopah Range recently demonstrated the benefits of using remotely piloted mobile robots in hazardous environments. This advanced robotic technology can be safely and effectively used to reduce the hazards typically associated with working in sites that contain unexploded ordnance and hazardous materials. RETRVIR's real-time sensing and control system make it an effective system to assist in the removal of over 100 million abandoned land mines worldwide and cleanup an estimated 3.1 million cubic meters of waste stored within the DOE complex. 


\title{
9.6 ION MOBILITY SPECTROMETRY (IMS) FOR CLASSIFICATION OF UXO
}

\section{Technical Contact}

\author{
Dr. Ronald L. Woodfin \\ Sandia National Laboratories \\ Org 2522 \\ Albuquerque, NM \\ Phone: (505) 844-3111 \\ Fax: (505) 844-7020 \\ E-mail: rlwoodf@sandia.gov

\section{Description of Technology}

Sandia is investigating the use of IMS technology as the detector section for explosive sensing systems. IMS technology can provide a positive indication of the presence or absence of explosives associated with a minelike object (MLO) or suspected unexploded ordnance (UXO). The technology can be made to work in both land and underwater applications. IMS was chosen because it provides a superior combination of sensitivity and specificity for the explosive molecules. Commercial units are available that approximate the required sensitivity, but are far from compact or rugged enough for military or humanitarian demining operations.

In use, a sample in either the liquid or gaseous phase is concentrated and desorbed into the ionized drift region of the IMS. The sample molecules pass through the drift region and are isolated based on the time required to register on the collector electrode. A microprocessor controls the system and can be gated to look for drugs, chemical agents, or, in this case, explosives.

\section{Application of Technology}

IMS technology is currently being used to sample for chemical agents, detect hidden drug packages, detect explosives in airport portals, identification of suspects, and forensics. Experimental work has shown that laboratory IMS equipment can detect extremely low levels of explosives from UXOs in bays and harbors. This IMS development program will use a miniaturized IMS designed by a local contractor and militarize it into a system that also concentrates the suspect analyte and provides an operator with go-no go indications rather than laboratory-style computer displays. The prototype unit will be designed to classify UXO from other nonexplosive objects in a sea water environment.

\section{Programmatics}

Funding for IMS sensor development has been obtained from the DOE/DoD MOU. The Office of Naval Research (ONR) is also funding similar and synergetic work, while additional work is being accomplished with internal Sandia (LDRD) funds. The program should result in demonstration of a first prototype of a semi-ruggedized IMS sensor system by the end of fiscal 1997. 


\subsection{USE OF RIGID POLYURETHANE FOAMS (RPF) IN MINE COUNTERMEASURES, CROSSING BEACH BARRIERS DURING AMPHIBIOUS ASSAULT, AND ROAD IMPROVEMENT AND REPAIR}

\section{Technical Contact}

Dr. Ronald L. Woodfin

Org 2522

Sandia National Laboratories

Albuquerque, NM

Phone: (505) 844-3111

Fax: (505) 844-7020,

E-mail: rlwoodf@sandia.gov

\section{Description of Technology}

Anti-personnel (AP) mines form a major hazard to troops in wartime. The Marine Corps encounters these mines in all the normal military uses but, in addition, finds them in the barriers that form a major part of beach defenses. The development of this application of RPFs was directed at neutralizing those mines and barriers simultaneously, permitting the Marine Corps vehicles to simply drive over the mined obstacles during an amphibious assault. We have shown that these materials, in properly chosen densities, can be used to defeat both the mines and the passive barriers. The foam is deployed as a two component liquid system where each component has a specific gravity near 1.0. The components are mixed and expand to the design density, normally in the range of 2 to 8 pounds per cubic foot (pcf), expanding 10 to 30 times the original volume.

\section{Application of Technology}

RPFs can carry a substantial traffic load and they can be deployed quickly under difficult conditions. As a byproduct of this study, we have determined that these materials can also be used to construct roadways over difficult terrain, such as beaches, ditches, tank traps, mudflats, and swamps and they have the potential for temporary (up to several months) repair of pavements subject to heavy traffic. RPFs can be deployed under a wide range of temperature and humidity conditions. In water, they expand to the design density and float to the surface, whereupon it becomes possible to pump sufficient material to build a roadway, bridge, or pier as required. On beach sand or mud, they can provide a surface capable of supporting heavy vehicles. Similarly, they can be used to quickly fill in a ditch or tank trap to permit vehicles to cross in a few minutes.

When applied over mines, the pressure transmitted to the mine firing device is greatly reduced. With appropriate choice of parameters, the pressure can be reduced to below that required to cause the mine to fire. Some types of anti-invasion mines use tilt rod firing devices that can be immobilized by encapsulating them in RPF, allowing them to be removed for disposal elsewhere. We have begun experiments to quantify the explosive energy absorbing capability of RPFs.

Several specific uses have been identified and are being developed:

1. construction of ramps, from the water onto the beach, covering the mined barriers erected to defend against amphibious assault

2. construction of a floating base course and roadway over water

3. construction of roadways over difficult terrain (tundra, swamp)

4. construction of temporary vehicle crossings over ditches and tank traps

5. construction of coverings over AP minefields to prevent mine explosions 
6. rapid repair of bomb and shell craters or pothole damaged pavements/runways

7. immobilization of the firing mechanisms on certain types of mines

8. absorption of part or all of the blast energy from mines that do explode

9. on-site construction of prefabricated floating causeways and repair of mine damaged causeways

\title{
Programmatics
}

The investigation into the military utility of RPF foam is being funded under the joint DOE/DoD MOU at approximately $\$ 500 \mathrm{~K}$ per year. A Technology Coordination Group with representatives from the interested DoD agencies meets semi-annually to provide oversight for the experimental effort. Considerable interest has been expressed by the Navy's NAVFAC Engineering Center and the Army Logistic Agency. The current laboratory work and field experiments are expected to result in demonstrations for the Services in late 1998/early 1999.

\subsection{Coded Aperture X-Ray Land Mine Imaging}

\section{Technical Contact}

\author{
Dr. Paul D. Rockett
}

Org. 2527

Sandia National Laboratories

Albuquerque, NM

Phone: (505) 845-7466

Fax: (505) 844-7020

E-mail: pdrocke@sandia.gov

\section{Description of Technology}

Coded aperture x-ray imaging uses a two-dimensional array of pinholes to image x-rays with the high angular resolution of a single pinhole, but has the large signal collection efficiency of hundreds of pinholes. Thus, high spatial resolution in the image is achieved without having to employ a high-power x-ray source.

Additionally, the reconstructed images are three-dimensional, showing depth as well as spatial location. By knowing the order of the array of pinholes, some open and some closed, one can use an inverse mathematical transformation to reconstruct the image. This technique replaces prior concepts that used large segments of a scintillator with very small spatial resolution and which usually required very large x-ray sources.

\section{Application of Technology}

The coded aperture will consist of several hundred pinholes in an approximate 15-cm square array, capable of providing a 4-mm resolution at the target in the ground. It will be primarily useful for land mine detection and imaging, regardless of whether the mine is plastic or metal. A fan beam source of X-rays from a portable Xray tube will illuminate the earth and the coded aperture detector will view the direct backscattered $\mathrm{x}$-rays. Though the fraction of x-ray photons backscattered will be small, the large area of the source and the sensitive detection screen will permit adequate image formation in a short time. Field of view may be 50 to 70 $\mathrm{cm}$ in diameter on the ground; however, this is only limited by the size of the detector and could easily grow by factors of 2 to 3 . 


\section{Programmatics}

Coded apertures are a common technology in medical imaging in nuclear medicine, in satellite-borne $\mathrm{x}$-ray telescopes, and in laser fusion applications. NASA has jointly flown satellites with the Danish Space Research Agency and with the Japanese Ministry of Industry and Technology. The DOE continues to fund research using X-ray coded apertures into the physics of laser-induced fusion for above ground testing applications at Lawrence Livermore National Laboratory with the support of Sandia. Coded apertures have been under development since the late 1960s and have become a mature field of endeavor. Mathematical reconstruction techniques have improved dramatically over the years to produce images with minimal artifacts and high signal-to-noise ratios. This research will build upon the existing Sandia program on X-Ray Backscatter Imaging, which has developed techniques for separating the return image from the ground surface from that below the surface.

\subsection{Transportable, Contained, Batch Destruction System for UXO}

\section{Technical Contact}

Brent Haroldsen

Phone: (510) 294-2590

Fax: (510) 294-1217

E-mail: blharol@sandia.gov

\section{Description of Technology}

Sandia is developing a transportable emergency destruction system that can be used to destroy unexploded ordinance in a fully-contained, non-vented manner. The reusable system consists of a pressure vessel with associated hardware and diagnostic equipment. The ordinance is opened inside the sealed vessel with a combination of linear and conical shaped charges. The vessel is designed to contain both the overpressure and the fragments. Remaining explosives or hazardous organic compounds are then destroyed, while still contained in the vessel, by either chemical neutralization (base hydrolysis) or batch supercritical water oxidation (SCWO). For neutralization, the vessel with the ordinance and neutralizing chemicals, is heated to $100^{\circ} \mathrm{C}$ and agitated to ensure complete reaction. If SCWO is used, the water and oxidizer are added to the vessel and it is heated to between $450^{\circ} \mathrm{C}$ and $500^{\circ} \mathrm{C}$, producing a pressure of about $240 \mathrm{bar}(3500 \mathrm{psi})$. At these conditions, the explosives and organic compounds are readily dissolved and oxidized.

\section{Application of Technology}

The system is currently being developed for explosively configured chemical weapons. It will be used for ordinance as large as eight inch artillery. The system is particularly suited for recovered ordinance of unknown condition or ordinance that contains hazardous materials besides the explosives. Development work has also demonstrated that the SCWO technology can be used with multiple, small, energetic components. In this case, the explosives are auto-detonated by heating the vessel instead of using shaped charges to open them. 


\section{Programmatics}

This work is being funded by the US Army Project Manager for Non-stockpile Chemical Materiel (PM NSCM). Chemical neutralization is the baseline destruction method. SCWO is being studied as an alternative. Demonstration of a half scale vessel is scheduled for October 1997.

\subsection{AUTOMATED HAZARD SEPARATION FOR MUNITION DEMILITARIZATION}

\section{Technical Contact}

Lawrence P. Ray

Sandia National Laboratories

Albuquerque, NM 87185

Phone: (505)844-6736

Fax: (505)844-9010

E-mail: lpray@sandia.gov

\section{Description of Technology}

Sandia is developing automated systems for dismantling and demilitarizing munitions and munition components. These methods combine the technologies of robotics and abrasive waterjet cutting to improve operator safety and increase material recovery and reuse. Robot technology allows for precise and autonomous handling and manipulation of parts utilizing vision system and force control feedback. Abrasive waterjet cutting provides an effective means for separating internal hazardous sub-components, sectioning munitions or providing access to the encased explosive for chemical treatment or neutralization.

\section{Application of Technology}

Unexploded ordnance (UXO) typically consists of an aeroshell, electronic sub-assemblies related to fusing and firing, and explosive materials. It may exist in a variety of conditions, possibly with damage to both external and internal components. This raises issues of safety for personnel handling such ordnance. In order to safely dispose of UXO, the ordnance must be rendered safe and the explosive materials rendered nonenergetic or completely decomposed to a non-hazardous state. Automated hazard separation is applicable to the processes of manipulating the UXO and opening it for fuse removal or neutralization of the explosive. Operators can oversee the removal, positioning, and cutting operations without exposure, while real-time data from optical and force sensors can provide precise control. A low pressure, high flow abrasive waterjet can effectively cut through hard steel casings without causing

detonation of explosive material. Both technologies allow for systems to be built with "off-the-shelf" components for operations in the field.

\section{Programmatics}

Both robotic technology and abrasive waterjet cutting technology are being developed and demonstrated at Sandia with funding from the Department of Energy (DOE). This development work has led to the recent implementation of prototype robotic systems at the DOE's Pantex Plant for the disassembly and handling of various nuclear weapon components. In addition, an automated waterjet cutting system is currently being developed by Sandia for the Department of Defense (DoD) at McAlester Army Ammunition Plant. The initial 
phase of this project is focused on stockpiled, conventional 40MM rounds. The DoD project is supported by the DoD/DOE Memorandum of Understanding with the Office of Munitions through TCGIX.

\subsection{CHEMICAL TAGGING AND SIGNAL TRANSDUCTION}

\section{Technical Contact}

R. J. Simonson

Org 2522

Sandia National Laboratories

Albuquerque, NM

Phone: (505) 844-7599

E-mail: rjsimon@sandia.gov

\section{Description of Technology}

We are investigating means of producing chemical compounds (taggants) which can be dispersed in the environment wherever UXO, mines, or other hazardous materials may be found. These compounds will be designed to react with explosives or other analytes to yield products that have distinct fluorescent or luminescent signatures. After reaction, such signatures can then be remotely detected using existing or dedicated optical surveillance systems. The taggant species must be environmentally benign.

\section{Application of Technology}

Several means exist for detection of "mine-like objects" and other packages that may contain unexploded ordnance. However, determination of whether or not such objects are actually hazardous, i.e., contain explosives is difficult by remote or noncontact means. The proposed tagging technology will allow for chemical-to-optical signal transduction, thereby enabling the classification of detected objects by remote means.

\section{Programmatic Issues}

A three-year effort in this area is currently supported by DOE-sponsored internal funding at Sandia. 


\section{SAVANNAH RIVER TECHNOLOGY CENTER}

\subsection{BIOLOGICAL UXO INDICATOR}

"The US Army Communications and Electronics Command's, Night Vision \& Electronic Sensors Directorate (CECOM/NVESD) has funded scientists at the Savannah River Technology Center (SRTC) to develop the Microbial Mine Detection System (MMDS). This project will explore the possibility of employing microorganisms to detect land mines.

SRTC scientists believe that a cost effective, safe biosensor system for detecting land mines can be developed using naturally occurring and indigenous microorganisms as the primary detection source. SRTC research has shown that various microbial species are stimulated by nitrogen, TNT, DNT, nitrates, nitrites, nitrous oxide, and other chemical components of explosive materials. Several of the some 10,000 bacteria already existing in SRTC's extensive Subsurface Microbiology Culture Collection (SMCC), contain bacterial luciferin and bioluminesce when active or growing. SRTC scientists expect to identify those bacteria residing in the SMCC, that can also be found in the immediate vicinity of land mines. Those species can then be selected or grouped by their attraction to the off-gasses of explosives; e.g., TNT in land mines, or by their bioluminescence characteristics.

The dual characteristics of certain bacteria to biochemically sense explosive compounds while producing bioluminescence allows these selected microorganisms to become powerful biosensor detectors. These microorganisms are expected to react similarly to the way trained canines alert to land mine off-gasses and other explosives. Once identified, the microorganisms can be placed in a variety of platforms for field deployment. The resulting bioluminescent activation will cause the bacteria to glow, so the locations of mines can be readily detected by appropriate night observation devices.

The development of the MMDS is in its initial phase of data gathering. The primary objective of this research is to identify a few naturally occurring microorganisms that provide the best opportunity for detecting and marking land mine locations." 


\section{INTERNAL DISTRIBUTION}
1-5. R. M. Davis
6. E. C. Fox
7. J. E. Hardy
8. D. M. Hetrick
9-13. C. M. Smith

\author{
14. B. A. Worley \\ 15. Central Research Library \\ 16 ORNL Laboratory Records-RC \\ 17 ORNL Laboratory Records-OSTI
}

\section{EXTERNAL DISTRIBUTION}

18. T. Broach, Countermine Division, ATTN: AMSEL-RD-NV-CD, 10221 Burbeck Road, Ste. 430, Ft. Belvoir, VA 22660-5806

19. V. George, Sandia National Laboratories, Computational Initiatives, P.O. Box 5800, MS-1188, Albuquerque, NM 87185-1188

20. W. Windsor, Joint UXO Coordination Office, 10221 Burbeck Road, Ft. Belvoir, VA 22060-5806 